\title{
Well posedness of Lagrangian flows and continuity equations in metric measure spaces
}

\author{
Luigi Ambrosio, Dario Trevisan *
}

March 28, 2019

This is a post-peer-review, pre-copyedit version of an article published in ANALYSIS AND PDE'S. The final authenticated version is available online at:

http://dx.doi.org/10.2140/apde.2014.7.1179

\begin{abstract}
We establish, in a rather general setting, an analogue of DiPerna-Lions theory on wellposedness of flows of ODE's associated to Sobolev vector fields. Key results are a wellposedness result for the continuity equation associated to suitably defined Sobolev vector fields, via a commutator estimate, and an abstract superposition principle in (possibly extended) metric measure spaces, via an embedding into $\mathbb{R}^{\infty}$.

When specialized to the setting of Euclidean or infinite dimensional (e.g. Gaussian) spaces, large parts of previously known results are recovered at once. Moreover, the class of $\operatorname{RCD}(K, \infty)$ metric measure spaces introduced in [AGS11b] and object of extensive recent research fits into our framework. Therefore we provide, for the first time, wellposedness results for ODE's under low regularity assumptions on the velocity and in a nonsmooth context.
\end{abstract}

\section{Contents}

1 Introduction $\quad 2$

$\begin{array}{lll}2 & \text { Notation and abstract setup } & \mathbf{7}\end{array}$

2.1 Dirichlet form and Carré du champ . . . . . . . . . . . . . . . 7

2.2 Laplace operator and Markov semigroup . . . . . . . . . . . . . . . . . . . 9

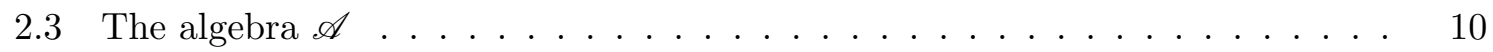

3 Derivations $\quad 12$

4 Existence of solutions to the continuity equation $\quad 14$

4.1 Auxiliary Hilbert spaces . . . . . . . . . . . . . . . . . . . . . 15

4.2 Existence under additional ellipticity . . . . . . . . . . . . . . . . 16

4.3 A priori estimates . . . . . . . . . . . . . . . . . 17

4.4 Vanishing viscosity and proof of Theorem $4.3 \ldots \ldots \ldots \ldots$

${ }^{*}$ Scuola Normale Superiore, Pisa, I.ambrosio@sns.it, dario.trevisan@sns.it 
5 Uniqueness of solutions to the continuity equation $\quad 22$

5.1 The commutator lemma . . . . . . . . . . . . . . 25

5.2 Proof of Theorem $5.4 \ldots \ldots \ldots \ldots \ldots \ldots \ldots$

6 Curvature assumptions and their implications $\quad 30$

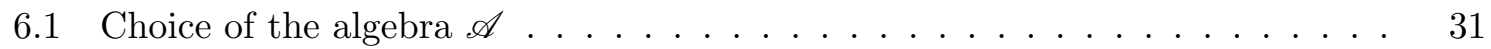

6.2 Conservation of mass . . . . . . . . . . . . . . . . . . 32

6.3 Derivations associated to gradients and their deformation . . . . . . . . . 32

7 The superposition principle in $\mathbb{R}^{\infty}$ and in metric measure spaces 35

8 Regular Lagrangian flows $\quad 41$

9 Examples $\quad 43$

9.1 Euclidean spaces: the DiPerna-Lions theory . . . . . . . . . . . . . . . . 43

9.2 Weighted Riemannian manifolds . . . . . . . . . . . . . . . 44

9.3 Abstract Wiener spaces . . . . . . . . . . . . . . . . . . . . . . . 45

9.4 Gaussian Hilbert spaces . . . . . . . . . . . . . . . . . . 46

9.5 Log-concave measures . . . . . . . . . . . . . . . . . . . 48

$9.6 \operatorname{RCD}(K, \infty)$ metric measure spaces . . . . . . . . . . . . . . . . 49

\section{Introduction}

The theory of DiPerna-Lions, initiated in the seminal paper [DL89], provides existence, stability and uniqueness results for ODE's associated to large classes of non-smooth vector fields, most notably that of Sobolev vector fields. In more recent times the first author extended in [Amb04] the theory to include $B V$ vector fields and, at the same time, he introduced a more probabilistic axiomatization based on the duality between flows and continuity equation, while the approach of [DL89] relied on characteristics and the transport equation. In more recent years the theory developed in many different directions, including larger classes of vector fields, quantitative convergence estimates, mild regularity properties of the flow, and non-Euclidean spaces, including infinite-dimensional ones. We refer to the Lecture Notes [Amb08] and [AC08] for more exhaustive, but still incomplete, description of the developments on this topic.

Aim of this paper is to extend the theory of well posedness for the continuity equation and the theory of flows to metric measure spaces $(X, d, \mathfrak{m})$. Roughly speaking, and obviously under additional structural assumptions, we prove that if $\left\{\boldsymbol{b}_{t}\right\}_{t \in(0, T)}$ is a time-dependent family of Sobolev vector fields then there is a unique flow associated to $\boldsymbol{b}_{t}$, namely a family of absolutely continuous maps $\{\boldsymbol{X}(\cdot, x)\}_{x \in X}$ from $[0, T]$ to $X$ satisfying:

(i) $\boldsymbol{X}(\cdot, x)$ solves the possibly non-autonomous ODE associated to $\boldsymbol{b}_{t}$ for $\mathfrak{m}$-a.e. $x \in X$;

(ii) the push-forward measures $\boldsymbol{X}(t, \cdot)_{\#} \mathfrak{m}$ are absolutely continuous w.r.t. $\mathfrak{m}$ and have uniformly bounded densities.

Of course the notions of "Sobolev vector field" and even "vector field", as well as the notion of solution to the ODE have to be properly understood in this nonsmooth context, where 
not even local coordinates are available. As far as we know, these are the first well-posedness results for ODE's under low regularity assumptions and in a nonsmooth context.

One motivation for writing this paper has been the theory of "Riemannian" metric measure spaces developed by the first author in collaboration with N. Gigli and G. Savaré, leading to a series of papers [AGS11a], [AGS11b], [AGS12] and to further developments in [Gig12], [Gig13]. In this perspective, it is important to develop new calculus tools in metric measure spaces. For instance, in the proof of the splitting theorem in [Gig13] a key role is played by the flow associated to the gradient of a $c$-concave harmonic function, whose flow lines provide the fibers of the product decomposition; therefore a natural question is under which regularity assumption on the potential $V$ the gradient flow associated to $V$ has a unique solution, where uniqueness is not understood pointwise, but in the sense of the DiPerna-Lions theory (see Theorem 8.3 and Theorem 9.6 for a partial answer to this question). We also point out the recent paper [GB14], where continuity equations in metric measure spaces are introduced and studied in connection with absolutely continuous curves with respect to the Wasserstein distance $W_{2}$, thus relying mainly on a "Lagrangian" point of view.

The paper is basically organized in three parts: in the "Eulerian" one, which has an independent interest, we study the well-posedness of continuity equations, in the "Lagrangian" one we define the notion of solution to the ODE and relate well-posedness of the continuity equation to existence and uniqueness of the flow (in the same spirit of [Amb04], [Amb08], where the context was Euclidean). Eventually, in the third part we see how a large class of previous results can be seen as particular cases of ours. On the technical side, these are the main ingredients: for the first part, a new intrinsic way to write down the so-called commutator estimate, obtained with $\Gamma$-calculus tools (this point of view is new even for "nice" spaces as Euclidean spaces and Riemannian manifolds); for the second part, a more general version of the so-called superposition principle (see for instance [AGS05, Theorem 8.2.1], in the setting of Euclidean spaces), that allows to lift, not canonically in general, nonnegative solutions to the continuity equation to measures on paths.

We pass now to a more detailed description of the three parts.

Part 1. This part consists of five sections, from Section 2 to Section 6 . Section 2 is devoted to the description of our abstract setup, which is the typical one of $\Gamma$-calculus and of the theory of Dirichlet forms: for the moment the distance is absent and we are given only a topology $\tau$ on $X$ and a reference measure $\mathfrak{m}$ on $X$, which is required to be Borel, nonnegative and $\sigma$-finite. On $L^{2}(\mathfrak{m})$ we are given a symmetric, densely defined and strongly local Dirichlet form $\mathcal{E}$ whose semigroup $\mathrm{P}$ is assumed to be Markovian. We also assume that a Carré du Champ $\Gamma: D(\mathcal{E}) \times D(\mathcal{E}) \rightarrow L^{1}(\mathfrak{m})$ is defined. Denoting by $\mathbb{V}$ the domain of $\mathcal{E}$, we assume that we are given a "nice" algebra $\mathscr{A}$ which is dense in $\mathbb{V}$ and which plays the role of the $C_{c}^{\infty}$ functions in the theory of distributions.

Using $\mathscr{A}$, we can define in Section 3 "vector fields" as derivations, in the same spirit of [We00] (see also [AK00] for parallel developments in the theory of currents): a derivation $\boldsymbol{b}$ is a linear map from $\mathscr{A}$ to the space of real-valued Borel functions on $X$, satisfying the Leibniz rule $\boldsymbol{b}(f g)=f \boldsymbol{b}(g)+g \boldsymbol{b}(f)$, and a pontwise $\mathfrak{m}$-a.e. bound in terms of $\Gamma$. We will use the more intuitive notation (since differentials of functions are co-vectors) $f \mapsto d f(\boldsymbol{b})$ for the action of a derivation $\boldsymbol{b}$ on $f$. An important example is provided by gradient derivations $\boldsymbol{b}_{V}$ induced by $V \in \mathbb{V}$ and acting as follows

$$
d f\left(\boldsymbol{b}_{V}\right):=\Gamma(V, f) .
$$

Although we will not need more than this, we would like to mention the forthcoming paper 
[Gig14], which provides equivalent axiomatizations, in which the Leibniz rule is not an axiom anymore, and it is shown that gradient derivations generate, in a suitable sense, all derivations. Besides the basic example of gradient derivations, the Carré du Champ provides, by duality, a natural pointwise norm on derivations; such duality can be used to define, via integration by parts, a notion of divergence $\operatorname{div} \boldsymbol{b}$ for a derivation (the divergence depends only on $\mathfrak{m}$, not on $\Gamma$ ). In Section 4 we prove existence of solutions to the weak formulation of the continuity equation $\frac{d}{d t} u_{t}+\operatorname{div}\left(u_{t} \boldsymbol{b}_{t}\right)=0$ induced by a family $\left(\boldsymbol{b}_{t}\right)$ of derivations, namely

$$
\frac{d}{d t} \int f u_{t} d \mathfrak{m}=\int d f\left(\boldsymbol{b}_{t}\right) u_{t} d \mathfrak{m} \quad \forall f \in \mathscr{A} .
$$

The strategy of the proof is classical: first we add a viscosity term and get a $\mathbb{V}$-valued solution by Hilbert space techniques, then we take a vanishing viscosity limit. Together with existence, we recover also higher (or lower, since our measure $\mathfrak{m}$ might be not finite and therefore no inclusion between $L^{p}$ spaces might hold) integrability estimates on $u$, depending on the initial condition $\bar{u}$. Also, under a suitable assumption (4.3) on $\mathscr{A}$, we prove that the $L^{1}$ norm is independent of time. Section 5 is devoted to the proof of uniqueness of solutions to the continuity equation. The classical proof is based on a smoothing scheme that, in our context, is played by the semigroup P (an approach already proved to be successful in [AF09], [Tre13], in Wiener spaces). For $t$ fixed, one has to estimate carefully the so-called commutator

$$
\mathscr{C}^{\alpha}\left(\boldsymbol{b}_{t}, u_{t}\right):=\operatorname{div}\left(\left(\mathrm{P}_{\alpha} u_{t}\right) \boldsymbol{b}_{t}\right)-\mathrm{P}_{\alpha}\left(\operatorname{div}\left(u_{t} \boldsymbol{b}_{t}\right)\right)
$$

as $\alpha \rightarrow 0$. The main new idea here is to imitate Bakry-Émery's $\Gamma$-calculus (see e.g. the recent monograph [BGL13]), interpolating and writing, at least formally,

$$
\begin{aligned}
\mathscr{C}^{\alpha}\left(\boldsymbol{b}_{t}, u_{t}\right) & =\int_{0}^{\alpha} \frac{d}{d s} \mathrm{P}_{\alpha-s}\left(\operatorname{div}\left(\mathrm{P}_{s}\left(u_{t}\right) \boldsymbol{b}_{t}\right)\right) d s \\
& =\int_{0}^{\alpha}\left[-\Delta \mathrm{P}_{\alpha-s}\left(\operatorname{div}\left(\mathrm{P}_{s}\left(u_{t}\right) \boldsymbol{b}_{t}\right)\right)+\mathrm{P}_{\alpha-s}\left(\operatorname{div}\left(\Delta \mathrm{P}_{s}\left(u_{t}\right) \boldsymbol{b}_{t}\right)\right)\right] d s .
\end{aligned}
$$

It turns out that an estimate of the commutator involves only the symmetric part of the derivative (this, in the Euclidean case, was already observed in [CP96] for regularizations induced by even convolution kernels). This structure can be recovered in our context: inspired by the definition of Hessian in [Bak94] we define the symmetric part D $\boldsymbol{c}^{\text {sym }}$ of the gradient a deformation $\boldsymbol{c}$ by

$$
\int D \boldsymbol{c}^{s y m}(f, g) d \mathfrak{m}:=-\frac{1}{2} \int d f(\boldsymbol{c}) \Delta g+d g(\boldsymbol{c}) \Delta f-(\operatorname{div} \boldsymbol{c}) \Gamma(f, g) d \mathfrak{m} .
$$

Then, we assume the validity of the estimate (see Definition 5.2 for a more general setup with different powers)

$$
\left|\int \operatorname{Dc}^{\text {sym }}(f, g) d \mathfrak{m}\right| \leq c\left(\int \Gamma(f)^{2} d \mathfrak{m}\right)^{1 / 4}\left(\int \Gamma(g)^{2} d \mathfrak{m}\right)^{1 / 4},
$$

which, in a smooth context, amount to an $L^{2}$ control on the symmetric part of derivative. Luckily, the control (1.3) on $D \boldsymbol{b}_{t}^{\text {sym }}$ can be combined with (1.1) to obtain strong convergence and therefore well-posedness of the continuity equation, assuming some regularizing properties of the semigroup $\mathrm{P}$, see Theorem 5.4. In particular, these hold assuming an abstract curvature 
lower bound on the underlying space, as discussed in Section 6, where we crucially exploit the recent results in [Sav13] and [AMS13] to show that our structural assumptions on $\mathrm{P}$ and on $\mathscr{A}$ are fulfilled in the presence of lower bounds on the curvature. Furthermore, gradient derivations associated to sufficiently regular functions satisfy (1.3).

Finally, we remark that, as in [DL89], analogous well-posedness results could be obtained for weak solutions to the inhomogeneous transport equation

$$
\frac{d}{d t} u_{t}+d u_{t}\left(\boldsymbol{b}_{t}\right)=c_{t} u_{t}+w_{t}
$$

under suitable assumptions on $c_{t}$ and $w_{t}$. We confined our discussion to the case of the homogeneous continuity equation (corresponding to $c_{t}=-\operatorname{div} \boldsymbol{b}_{t}$ and $w_{t}=0$ ) for the sake of simplicity and for the relevance of this PDE in connection with the theory of flows.

Part 2. This part consists of two sections. In Section 7 we show how solutions $u$ to the continuity equation $\frac{d}{d t} u_{t}+\operatorname{div}\left(u_{t} \boldsymbol{b}_{t}\right)=0$ can be lifted to measures $\boldsymbol{\eta}$ in $C([0, T] ; X)$. Namely, we would like that $\left(e_{t}\right)_{\#} \boldsymbol{\eta}=u_{t} \mathfrak{m}$ for all $t \in(0, T)$ and that $\boldsymbol{\eta}$ is concentrated on solutions $\eta$ to the ODE $\dot{\eta}=\boldsymbol{b}_{t}(\eta)$. This statement is well-known in Euclidean spaces (or even Hilbert spaces), see [AGS05, Thm. 8.2.1]; in terms of currents, it could be seen as a particular case of Smirnov's decomposition [Smi94] of 1-currents as superposition of rectifiable currents. Here we realized that the most appropriate setup for the validity of this principle is $\mathbb{R}^{\infty}$, see Theorem 7.1, where only the Polish structure of $\mathbb{R}^{\infty}$ matters and neither distance nor reference measure come into play.

In order to extend this principle from $\mathbb{R}^{\infty}$ to our abstract setup we assume the existence of a sequence $\left(g_{k}\right) \subset\left\{f \in \mathscr{A}:\|\Gamma(f)\|_{\infty} \leq 1\right\}$ satisfying:

$\operatorname{span}\left(g_{k}\right)$ is dense in $\mathbb{V}$ and any function $g_{k}$ is $\tau$-continuous,

$$
\exists \lim _{n \rightarrow \infty} g_{k}\left(x_{n}\right) \text { in } \mathbb{R} \text { for all } k \Longrightarrow \exists \lim _{n \rightarrow \infty} x_{n} \text { in } X .
$$

This way, the embedding $J: X \rightarrow \mathbb{R}^{\infty}$ mapping $x$ to $\left(g_{k}(x)\right)$ is provides an homeomorphism of $X$ with $J(X)$ and we can first read the solution to the continuity equation in $\mathbb{R}^{\infty}$ (setting $\nu_{t}:=J_{\#}\left(u_{t} \mathfrak{m}\right)$, with an appropriate choice of the velocity in $\left.\mathbb{R}^{\infty}\right)$ and then pull back the lifting obtained in $\mathscr{P}\left(C\left([0, T] ; \mathbb{R}^{\infty}\right)\right)$ to obtain $\boldsymbol{\eta} \in \mathscr{P}(C([0, T] ; X))$, see Theorem 7.6. It turns out that $\boldsymbol{\eta}$ is concentrated on curves $\eta$ satisfying

$$
\frac{d}{d t}(f \circ \eta)=d f\left(\boldsymbol{b}_{t}\right) \circ \eta \quad \text { in the sense of distributions in }(0, T) \text {, for all } f \in \mathscr{A},
$$

which is the natural notion of solution to the ODE $\dot{\eta}=\boldsymbol{b}_{t}(\eta)$ in our context (again, consistent with the fact that a vector can be identified with a derivation). We show, in addition, that this property implies absolute continuity of $\boldsymbol{\eta}$-almost every curve $\eta$ with respect to the possibly extended distance $d(x, y):=\sup _{k}\left|g_{k}(x)-g_{k}(y)\right|$, with metric derivative $|\dot{\eta}|$ estimated from above with $\left|\boldsymbol{b}_{t}\right| \circ \eta$. Notice also that, in our setup, the distance appears only now. Also, we remark that a similar change of variables appears in the recent paper [KR13], but not in a Lagrangian perspective: it is used therein to prove well-posedness of the continuity equation when the reference measure is log-concave (see Section 9.5).

Section 8 is devoted to the proof of Theorem 8.3, which links well-posedness of the continuity equation in the class of nonnegative functions $L_{t}^{1}\left(L_{x}^{1} \cap L_{x}^{\infty}\right)$ with initial data $\bar{u} \in L^{1} \cap L^{\infty}(\mathfrak{m})$ 
to the existence and uniqueness of the flow $\boldsymbol{X}$ according to (i), (ii) above, where (i) is now understood as in (1.6). The proof of Theorem 8.3 is based on two facts: first, the possibility to lift solutions $u$ to probabilities $\boldsymbol{\eta}$, discussed in the previous section; second, the fact that the restriction of $\boldsymbol{\eta}$ to any Borel set still induces a solution to the continuity equation with the same velocity field. Therefore we can "localize" $\boldsymbol{\eta}$ to show that, whenever some branching of trajectories occurs, then there is non-uniqueness at the level of the continuity equation.

Let us comment that, in this abstract setting, it seems more profitable to the authors to deal uniquely with continuity equations, instead of transport equations as in [DL89], since the latter require in its very definition a choice of "coordinates", while the former arises naturally as the description of evolution of underlying measures.

Part 3. This part consists of Section 9 only, where we specialize the general theory to settings where continuity equations and associated flows have already been considered, and to $\operatorname{RCD}(K, \infty)$-metric measure spaces. Since the transfer mechanism of well-posedness from the PDE to the ODE levels is quite general, we mainly focus on the continuity equation. Moreover, in these particular settings (except for $\operatorname{RCD}(K, \infty)$ spaces), the proof of existence for solutions turns out to be a much easier task than in the general framework, due to explicit and componentwise approximations by smooth vector fields. Therefore, we limit ourselves to compare uniqueness results.

In Section 9.1, we show how the classical DiPerna-Lions theory of [DL89] fits into our setting: in short, we recover almost all the well-posedness results in [DL89], with the notable exception of $W_{\text {loc }}^{1,1}$-regular vector fields. In Section 9.2 we also describe how our techniques provide intrinsic proofs, i.e. without reducing to local coordinates, of analogous results for weighted Riemannian manifolds.

In Section 9.3 and Section 9.4, we deal with (infinite dimensional) Gaussian frameworks, comparing our results to those established respectively in [AF09], [DFR13]: large parts of these can be obtained as consequences of our general theory, which turns out to be more flexible e.g. we can allow for vector fields that do not necessarily take values in the CameronMartin space (see at the end of Section 9.4), which is not admissible in [AF09] or in [DFR13]. In Section 9.5 we consider the even more general setting of log-concave measures and make a comparison with some of the results contained in [KR13]. The strength of our approach is immediately revealed, e.g. we are not limited as in [KR13] to uniformly log-concave measures.

We conclude in Section 9.6 by describing how the theory specializes to the setting of $\operatorname{RCD}(K, \infty)$-metric measure spaces, that is one of our original motivations for this work. We show that Lagrangian flows do exist in many cases (Theorem 9.6) and provide instances of so-called test plans. In the case of gradient derivations, we also show that the trajectories satisfy a global energy dissipation identity (Theorem 9.5).

Acknowledgments. The first author acknowledges the support of the ERC ADG GeMeThNES. The second author has been partially supported by PRIN10-11 grant from MIUR for the project Calculus of Variations. Both authors are members of the GNAMPA group of the Istituto Nazionale di Alta Matematica (INdAM).

The authors thank G. Savaré for pointing out to them, after reading a preliminary version of this work, the possibility to dispense from the full curvature assumption, an observation that led to the present organization of the paper. They also thank F. Ricci and G. Da Prato for useful discussions. 


\section{Notation and abstract setup}

Let $(X, \tau)$ be a Polish topological space, endowed with a $\sigma$-finite Borel measure $\mathfrak{m}$ with full support (i.e. $\operatorname{supp} \mathfrak{m}=X$ ) and

a strongly local, densely defined and symmetric Dirichlet form $\mathcal{E}$ on $L^{2}(X, \mathscr{B}(X), \mathfrak{m})$

enjoying a Carré du Champ $\Gamma: D(\mathcal{E}) \times D(\mathcal{E}) \rightarrow L^{1}(X, \mathscr{B}(X), \mathfrak{m})$ and

generating a Markov semigroup $\left(\mathrm{P}_{t}\right)_{t \geq 0}$ on $L^{2}(X, \mathscr{B}(X), \mathfrak{m})$.

To keep notation simple, we write $L^{p}(\mathfrak{m})$ instead of $L^{p}(X, \mathscr{B}(X), \mathfrak{m})$ and denote $L^{p}(\mathfrak{m})$ norms by $\|\cdot\|_{p}$. We also write $L^{0}(\mathfrak{m})$ for the space of $\mathfrak{m}$-a.e. equivalence classes of Borel functions $f: X \mapsto[-\infty,+\infty]$ that take finite values $\mathfrak{m}$-a.e. in $X$.

Since $(X, \tau)$ is Polish and $\mathfrak{m}$ is $\sigma$-finite, the spaces $L^{p}(\mathfrak{m})$ are separable for $p \in[1, \infty)$. We shall also use the duality relations

$$
\left(L^{p}(\mathfrak{m})+L^{q}(\mathfrak{m})\right)^{*}=L^{p^{\prime}} \cap L^{q^{\prime}}(\mathfrak{m}) \quad \text { p, } q \in[1, \infty)
$$

and the notation $\|\cdot\|_{L^{p}+L^{q}},\|\cdot\|_{L^{p^{\prime}} \cap L^{q^{\prime}}}$. In addition, we will use that the spaces $L^{p}(\mathfrak{m})$, $1 \leq p \leq \infty$ (and $p=0$ ) are complete lattices with respect to the order relation induced by the inequality $\mathfrak{m}$-a.e. in $X$. This follows at once from the general fact that, for any family of Borel functions $f_{i}: X \rightarrow[-\infty,+\infty]$ there exists $f: X \rightarrow[-\infty,+\infty]$ Borel such that $f \geq f_{i}$ $\mathfrak{m}$-a.e. in $X$ for all $i \in I$ and $f \geq g \mathfrak{m}$-a.e. in $X$ for any function $g$ with the same property. Existence of $f$ can be achieved, for instance, by considering the maximization of

$$
J \mapsto \int \tan ^{-1}\left(\sup _{i \in J} f_{i}\right) \vartheta d \mathfrak{m}
$$

among the finite subfamilies $J$ of $I$, with $\vartheta$ positive function in $L^{1}(\mathfrak{m})$.

Now, let us briefly recall the precise meaning of (2.1).

\subsection{Dirichlet form and Carré du champ}

A symmetric Dirichlet form $\mathcal{E}$ is a $L^{2}(\mathfrak{m})$-lower semicontinuous quadratic form satisfying the Markov property

$$
\mathcal{E}(\eta \circ f) \leq \mathcal{E}(f) \quad \text { for every normal contraction } \eta: \mathbb{R} \rightarrow \mathbb{R},
$$

i.e. a 1-Lipschitz map satisfying $\eta(0)=0$. We refer to [BH91, FOT11] for equivalent formulations of (2.2). Recall that

$$
\mathbb{V}:=\mathrm{D}(\mathcal{E}) \subset L^{2}(\mathfrak{m}), \quad \text { endowed with }\|f\|_{\mathbb{V}}^{2}:=\int_{X}\left(f^{2}+\Gamma(f)\right) d \mathfrak{m}
$$

is a Hilbert space. Furthermore, $\mathbb{V}$ is separable because $L^{2}(\mathfrak{m})$ is separable (see [AGS11b, Lemma 4.9] for the simple proof).

We still denote by $\mathcal{E}(\cdot, \cdot): \mathbb{V} \rightarrow \mathbb{R}$ the associated continuous and symmetric bilinear form

$$
\mathcal{E}(f, g):=\frac{1}{4}(\mathcal{E}(f+g)-\mathcal{E}(f-g)) .
$$


We will assume strong locality of $\mathcal{E}$, namely

$$
\forall f, g \in \mathbb{V}: \quad \mathcal{E}(f, g)=0, \quad \text { if }(f+a) g=0 \mathfrak{m} \text {-a.e. in } X \text { for some } a \in \mathbb{R} \text {. }
$$

It is possible to prove (see for instance [BH91, Prop. 2.3.2]) that $\mathbb{V} \cap L^{\infty}(\mathfrak{m})$ is an algebra with respect to pointwise multiplication, so that for every $f \in \mathbb{V} \cap L^{\infty}(\mathfrak{m})$ the linear form on $\mathbb{V} \cap L^{\infty}(\mathfrak{m})$

$$
\Gamma[f ; \varphi]:=2 \mathcal{E}(f, f \varphi)-\mathcal{E}\left(f^{2}, \varphi\right), \quad \varphi \in \mathbb{V} \cap L^{\infty}(\mathfrak{m}),
$$

is well defined and, for every normal contraction $\eta: \mathbb{R} \rightarrow \mathbb{R}$, it satisfies [BH91, Prop. 2.3.3]

$$
0 \leq \Gamma[\eta \circ f ; \varphi] \leq \Gamma[f ; \varphi] \leq\|\varphi\|_{\infty} \mathcal{E}(f) \quad \text { for all } f, \varphi \in \mathbb{V} \cap L^{\infty}(\mathfrak{m}), \varphi \geq 0 .
$$

The inequality (2.4) shows that for every nonnegative $\varphi \in \mathbb{V} \cap L^{\infty}(\mathfrak{m})$ the function $f \mapsto \Gamma[f ; \varphi]$ is a quadratic form in $\mathbb{V} \cap L^{\infty}(\mathfrak{m})$ which satisfies the Markov property and can be extended by continuity to $\mathbb{V}$.

We assume that for all $f \in \mathbb{V}$ the linear form $\varphi \mapsto \Gamma[f ; \varphi]$ can be represented by a an absolutely continuous measure w.r.t. $\mathfrak{m}$ with density $\Gamma(f) \in L_{+}^{1}(\mathfrak{m})$, the so-called Carré $d u$ champ. Since $\mathcal{E}$ is strongly local, [BH91, Thm. 6.1.1] yields the representation formula

$$
\mathcal{E}(f, f)=\int_{X} \Gamma(f) d \mathfrak{m}, \quad \text { for all } f \in \mathbb{V} .
$$

It is not difficult to check that $\Gamma$ as defined by (2.5) (see e.g. [BH91, Def. 4.1.2]) is a quadratic continuous map defined in $\mathbb{V}$ with values in $L_{+}^{1}(\mathfrak{m})$, and that $\Gamma[f-g ; \varphi] \geq 0$ for all $\varphi \in$ $\mathbb{V} \cap L^{\infty}(\mathfrak{m})$ yields

$$
|\Gamma(f, g)| \leq \sqrt{\Gamma(f)} \sqrt{\Gamma(g)}, \quad \mathfrak{m} \text {-a.e. in } X .
$$

We use the $\Gamma$ notation also for the symmetric, bilinear and continuous map

$$
\Gamma(f, g):=\frac{1}{4}(\Gamma(f+g)-\Gamma(f-g)) \in L^{1}(\mathfrak{m}) \quad f, g \in \mathbb{V},
$$

which, thanks to (2.5), represents the bilinear form $\mathcal{E}$ by the formula

$$
\mathcal{E}(f, g)=\frac{1}{2} \int_{X} \Gamma(f, g) d \mathfrak{m} \text { for all } f, g \in \mathbb{V} .
$$

Because of Markovianity and locality, $\Gamma(\cdot, \cdot)$ satisfies the chain rule [BH91, Cor. 7.1.2]

$$
\Gamma(\eta(f), g)=\eta^{\prime}(f) \Gamma(f, g) \quad \text { for all } f, g \in \mathbb{V}, \eta: \mathbb{R} \rightarrow \mathbb{R} \text { Lipschitz with } \eta(0)=0,
$$

and the Leibniz rule:

$$
\Gamma(f g, h)=f \Gamma(g, h)+g \Gamma(f, h) \quad \text { for all } f, g, h \in \mathbb{V} \cap L^{\infty}(\mathfrak{m}) .
$$

Notice that by [BH91, Thm. 7.1.1] (2.7) is well defined, since for every Borel set $N \subset \mathbb{R}$ (as the set where $\eta$ is not differentiable) there holds

$$
\mathscr{L}^{1}(N)=0 \quad \Rightarrow \quad \Gamma(f)=0 \quad \mathfrak{m} \text {-a.e. on } f^{-1}(N) .
$$


For $p \in[1, \infty]$, we introduce the spaces

$$
\mathbb{V}_{p}:=\left\{u \in \mathbb{V} \cap L^{p}(\mathfrak{m}): \int(\Gamma(u))^{p / 2} d \mathfrak{m}<\infty\right\} \quad p \in[1, \infty),
$$

with the obvious extension to $p=\infty$. As in $\left[\right.$ BH91, §6.2], one can endow each $\mathbb{V}_{p}$ with the norm

$$
\|f\|_{\mathbb{V}_{p}}=\|f\|_{\mathbb{V}}+\|f\|_{p}+\left\|\Gamma(u)^{1 / 2}\right\|_{p},
$$

thus obtaining a Banach space, akin to the intersection of classical Sobolev spaces $W^{1,2} \cap W^{1, p}$. Notice that $\mathbb{V}_{2}=\mathbb{V}$, with an equivalent norm. The Banach space structure plays a major role only starting from Section 5 , but the notation $f \in \mathbb{V}_{p}$ is conveniently used throughout.

\subsection{Laplace operator and Markov semigroup}

The Dirichlet form $\mathcal{E}$ induces a densely defined, negative and selfadjoint operator $\Delta: D(\Delta) \subset$ $\mathbb{V} \rightarrow L^{2}(\mathfrak{m})$, defined by the integration by parts formula $\mathcal{E}(f, g)=-\int_{X} g \Delta f d \mathfrak{m}$ for all $g \in \mathbb{V}$. The operator $\Delta$ is of "diffusion" type, since it satisfies the following chain rule for every $\eta \in C^{2}(\mathbb{R})$ with $\eta(0)=0$ and bounded first and second derivatives (see [BH91, Corollary 6.1.4]): whenever $f \in D(\Delta)$ with $\Gamma(f) \in L^{2}(\mathfrak{m})$, then $\eta(f) \in D(\Delta)$ and

$$
\Delta \eta(f)=\eta^{\prime}(f) \Delta f+\eta^{\prime \prime}(f) \Gamma(f) .
$$

The "heat flow" $\mathrm{P}_{t}$ associated to $\mathcal{E}$ is well defined starting from any initial condition $f \in L^{2}(\mathfrak{m})$. Recall that in this framework the heat flow $\left(\mathrm{P}_{t}\right)_{t \geq 0}$ is an analytic Markov semigroup and that $f_{t}=\mathrm{P}_{t} f$ can be characterized as the unique $C^{1}$ map $f:(0, \infty) \rightarrow L^{2}(\mathfrak{m})$, with values in $D(\Delta)$, satisfying

$$
\begin{cases}\frac{d}{d t} f_{t}=\Delta f_{t} & \text { for } t \in(0, \infty), \\ \lim _{t \downarrow 0} f_{t}=f & \text { in } L^{2}(\mathfrak{m}) .\end{cases}
$$

Because of this, $\Delta$ can equivalently be characterized in terms of the strong convergence $\left(\mathrm{P}_{t} f-f\right) / t \rightarrow \Delta f$ in $L^{2}(\mathfrak{m})$ as $t \downarrow 0$.

Furthermore, we have the regularization estimates (in the more general context of gradient flows of convex functionals, see for instance [AGS05, Thm. 4.0.4(ii)])

$$
\begin{aligned}
& \mathcal{E}\left(\mathrm{P}_{t} u, \mathrm{P}_{t} u\right) \leq \inf _{v \in \mathbb{V}}\left\{\mathcal{E}(v, v)+\frac{\|v-u\|_{2}^{2}}{2 t}\right\}<\infty, \quad \forall t>0, u \in L^{2}(\mathfrak{m}), \\
& \left\|\Delta \mathrm{P}_{t} u\right\|_{2}^{2} \leq \inf _{v \in D(\Delta)}\left\{\|\Delta v\|_{2}^{2}+\frac{\|v-u\|_{2}^{2}}{t^{2}}\right\}<\infty, \quad \forall t>0, u \in L^{2}(\mathfrak{m}) .
\end{aligned}
$$

For $p \in(1, \infty)$, we shall also need an $L^{p}$ version of $(2.13)$, namely

$$
\left\|\Delta \mathrm{P}_{t} f\right\|_{p} \leq \frac{c_{p}^{\Delta}}{t}\|f\|_{p}, \quad \text { for every } f \in L^{p} \cap L^{2}(\mathfrak{m}) \text { and every } t \in(0,1) .
$$

This can be obtained as a consequence of the fact that $\mathrm{P}$ is analytic [Ste70, Thm. II.1]: it is actually equivalent to it, see [Yos80, §X.10].

As an easy corollary of (2.14), we obtain the following estimate. 
Corollary 2.1. Let $p \in(1, \infty)$ and let $c_{p}^{\Delta}$ be the constant in (2.14). Then

$$
\left\|\mathrm{P}_{t} f-\mathrm{P}_{t-t^{\prime}} f\right\|_{p} \leq \min \left\{c_{p}^{\Delta} \log \left(1+\frac{t^{\prime}}{t-t^{\prime}}\right), 2\right\}\|f\|_{p}, \quad \forall f \in L^{p} \cap L^{2}(\mathfrak{m})
$$

for every $t, t^{\prime} \in(0,1)$, with $t^{\prime} \leq t$.

Proof. The estimate with the constant 2 follows from $L^{p}$ contractivity. For the other one, we apply (2.14) as follows:

$$
\left\|\mathrm{P}_{t} f-\mathrm{P}_{t-t^{\prime}} f\right\|_{p} \leq \int_{0}^{t^{\prime}}\left\|\Delta \mathrm{P}_{t-t^{\prime}+r} f\right\|_{p} d r \leq \int_{0}^{t^{\prime}} \frac{c_{p}^{\Delta}}{t-t^{\prime}+r} d r\|f\|_{p}=c_{p}^{\Delta} \log \left(1+\frac{t^{\prime}}{t-t^{\prime}}\right)\|f\|_{p} .
$$

One useful consequence of the Markov property is the $L^{p}$ contraction of $\left(\mathrm{P}_{t}\right)_{t \geq 0}$ from $L^{p} \cap L^{2}$ to $L^{p} \cap L^{2}$. Because of the density of $L^{p} \cap L^{2}$ in $L^{p}$ when $p \in[1, \infty)$, this allows to extend uniquely $\mathrm{P}_{t}$ to a strongly continuous semigroup of linear contractions in $L^{p}(\mathfrak{m})$, $p \in[1, \infty)$, for which we retain the same notation. Furthermore, $\left(\mathrm{P}_{t}\right)_{t \geq 0}$ is sub-Markovian (cf. [BH91, Prop. 3.2.1]), since it preserves one-sided essential bounds, namely $f \leq C$ (resp. $f \geq C$ ) $m$-a.e. in $X$ for some $C \geq 0$ (resp. $C \leq 0$ ) implies $\mathrm{P}_{t} f \leq C$ (resp. $\mathrm{P}_{t} f \geq C$ ) $\mathfrak{m}$-a.e. in $X$ for all $t \geq 0$.

Finally, it is easy to check, using $L^{1}$-contractivity of $\mathrm{P}$, that the dual semigroup $\mathrm{P}_{t}^{\infty}$ : $L^{\infty}(\mathfrak{m}) \rightarrow L^{\infty}(\mathfrak{m})$ given by

$$
\int g \mathrm{P}_{t}^{\infty} f d \mathfrak{m}=\int f \mathrm{P}_{t} g d \mathfrak{m}, \quad f \in L^{\infty}(\mathfrak{m}), g \in L^{1}(\mathfrak{m})
$$

is well defined. It is a contraction semigroup in $L^{\infty}(\mathfrak{m})$, sequentially $w^{*}$-continuous, and it coincides with $\mathrm{P}$ on $L^{2} \cap L^{\infty}(\mathfrak{m})$.

\subsection{The algebra $\mathscr{A}$}

Throughout the paper we assume that an algebra $\mathscr{A} \subset \mathbb{V}$ is prescribed, with

$$
\mathscr{A} \subset \bigcap_{p \in[1, \infty]} L^{p}(\mathfrak{m}), \quad \mathscr{A} \text { dense in } \mathbb{V}
$$

and

$$
\Phi\left(f_{1}, \ldots, f_{n}\right) \in \mathscr{A} \quad \text { whenever } \Phi \in C^{1}\left(\mathbb{R}^{n}\right), f_{1}, \ldots, f_{n} \in \mathscr{A} .
$$

Additional conditions on $\mathscr{A}$ will be considered in specific sections of the paper. A particular role is played by the condition $\mathscr{A} \subset \mathbb{V}_{p}$, for $p \in[2, \infty]$. By interpolation, if such an inclusion holds, then it holds for every $q$ between 2 and $p$. About the inclusion $\mathscr{A} \subset \mathbb{V}_{p}$ for $p \in[1,2]$, we prove the following lemma.

Lemma 2.2. Let $\mathcal{A} \subset \mathbb{V}$ be dense in $\mathbb{V}$ and satisfy (2.17). Then, there exists $\mathscr{A} \subset \mathcal{A}$, such that (2.16) and (2.17) hold, and

$$
\mathscr{A} \text { is contained and dense in } \mathbb{V}_{p} \text {, for every } p \in[1,2] \text {. }
$$

In particular, without any loss of generality, we assume throughout that (2.18) holds. 
Proof. We define

$$
\mathscr{A}=\{\Phi(f): f \in \mathcal{A}, \Phi \in \mathcal{F}\} \subset \mathcal{A},
$$

where $\mathcal{F}$ consists of all functions $\Phi: \mathbb{R} \rightarrow \mathbb{R}$ bounded and Lipschitz, continuously differentiable and null at the origin, with $\Phi^{\prime}(x) / x$ bounded in $\mathbb{R}$. By the chain rule and Hölder inequality, it follows that $\mathscr{A} \subset L^{p}(\mathfrak{m})$ for all $p \in[1, \infty]$ and that $(2.17)$ holds. We address the density of $\mathscr{A}$ in $\mathbb{V}_{p}$ for $p \in[1,2]$.

We consider Lipschitz functions $\phi_{n}: \mathbb{R} \mapsto[0,1]$ such that $\phi_{n}(z)=0$ for $|z| \leq 1 /(2 n)$ and for $|z| \geq 2 n$, while $\phi_{n}(z)=1$ for $|z| \in[1 / n, n]$, and we set $\Phi_{n}(z)=\int_{0}^{z} \phi_{n}(t) d t$. Notice that $\Phi_{n} \equiv 0$ on $[-1 /(2 n), 1 /(2 n)]$, that $\Phi_{n}$ belongs to $\mathcal{F}$, and that $\Phi_{n}^{\prime}(z)=\phi_{n}(z) \rightarrow 1$ as $n \rightarrow \infty$. It is easily seen, by chain rule, that $\Phi_{n}(f) \rightarrow f$ in $\mathbb{V}_{p}$ as $n \rightarrow \infty$ for all $f \in \mathbb{V}_{p}$, therefore density is achieved if we show that all functions $\Phi_{n}(f)$ belong to the closure of $\mathscr{A}$. Since by assumption there exist $f_{k} \in \mathcal{A}$ convergent to $f$ in $\mathbb{V}$, it will be sufficient to show that $\Phi_{n}\left(f_{k}\right)$ converge to $\Phi_{n}(f)$ in $\mathbb{V}_{p}$.

We claim that $\phi_{n}\left(f_{k}\right)$ converge to $\phi(f)$ in $L^{q}(\mathfrak{m})$ for all $q \in[1, \infty)$. To prove the claim, it suffices to consider separately the sets $\{|f| \geq 1 /(3 n)\}$ and $\{|f|<1 /(3 n)\}$. On the first set, which has finite $\mathfrak{m}$-measure, we can use dominated convergence to achieve convergence, taking the boundedness of $\phi_{n}$ into account; on the second set we have

$$
\left|\phi_{n}\left(f_{k}\right)-\phi_{n}(f)\right|=\chi_{\left\{\left|f_{k}\right| \geq 1 /(2 n)\right\}}\left|\phi_{n}\left(f_{k}\right)-\phi_{n}(f)\right| \leq \chi_{\left\{\left|f_{k}-f\right| \geq 1 /(6 n)\right\}} \min \left\{2, \operatorname{Lip}\left(\phi_{n}\right)\left|f_{k}-f\right|\right\}
$$

and we can use Hölder's inequality for $q<2$ and uniform boundedness for $q \geq 2$.

To show convergence in $\mathbb{V}_{p}$, we use the following straightforward identity, valid for any $h_{1}, h_{2} \in \mathbb{V}$ and $\Phi \in \mathcal{F}$ :

$\Gamma\left(\Phi\left(h_{1}\right)-\Phi\left(h_{2}\right)\right)=\left(\Phi^{\prime}\left(h_{1}\right)-\Phi^{\prime}\left(h_{2}\right)\right)^{2} \Gamma\left(h_{1}, h_{2}\right)+\Phi^{\prime}\left(h_{1}\right)^{2} \Gamma\left(h_{1}, h_{1}-h_{2}\right)+\Phi^{\prime}\left(h_{2}\right)^{2} \Gamma\left(h_{2}, h_{2}-h_{1}\right)$.

Adding and subtracting $\Phi^{\prime}\left(h_{2}\right)^{2} \Gamma\left(h_{1}, h_{1}-h_{2}\right)$, and taking $\Phi=\Phi_{n}$, since $0 \leq \phi_{n} \leq 1$ we obtain the inequality

$$
\begin{aligned}
\Gamma\left(\Phi_{n}\left(h_{1}\right)-\Phi_{n}\left(h_{2}\right)\right)^{1 / 2} \leq & \left|\phi_{n}\left(h_{1}\right)-\phi_{n}\left(h_{2}\right)\right| \Gamma\left(h_{1}\right)^{1 / 4} \Gamma\left(h_{2}\right)^{1 / 4} \\
& \left.+2 \mid \phi_{n}\left(h_{1}\right)-\phi_{n}\left(h_{2}\right)\right)\left.\right|^{1 / 2} \Gamma\left(h_{1}\right)^{1 / 4} \Gamma\left(h_{1}-h_{2}\right)^{1 / 4} \\
& +\phi_{n}\left(h_{2}\right) \Gamma\left(h_{1}-h_{2}\right)^{1 / 2}
\end{aligned}
$$

We take $h_{1}=f$ and $h_{2}=f_{k}$ above and using the convergence of $\phi_{n}\left(f_{k}\right)$ to $\phi_{n}(f)$ in every $L^{q}(\mathfrak{m})$ space, for $q \in[1, \infty)$, as well as Hölder's inequality, it is easy to check that the right hand side above converge to 0 in $L^{p}(\mathfrak{m})$.

We also deduce density in $L^{p} \cap L^{q}$-spaces, thanks to the following lemma.

Lemma 2.3. There exists a countable set $\mathscr{D} \subset \mathscr{A}$ dense in $L^{p} \cap L^{q}(\mathfrak{m}), 1 \leq p \leq q<\infty$, and $w^{*}$-dense in $L^{\infty}(\mathfrak{m})$.

Proof. Since $\mathbb{V}$ is dense in $L^{2}(\mathfrak{m})$ and we are assuming that $\mathscr{A}$ is dense in $\mathbb{V}$, we obtain that $\mathscr{A}$ is dense in $L^{2}(\mathfrak{m})$.

We consider first the case $p=q \in[2, \infty]$. Let $h \in L^{p^{\prime}}(\mathfrak{m})$. Assuming $\int h \varphi d \mathfrak{m}=0$ for all $\varphi \in \mathscr{A}$, to prove density in the $w^{*}$ topology (and then in the strong topology if $p<\infty$ ) we have to prove that $h=0$. Let $\delta>0$, set $f_{\delta}=\operatorname{sign} h \chi_{\{|h|>\delta\}}$ (set equal to 0 wherever $h=0)$ and find an equibounded sequence $\left(\varphi_{n}\right) \subset \mathscr{A}$ convergent in $L^{2}(\mathfrak{m})$ to $f_{\delta}$. Since $\left(\varphi_{n}\right)$ 
are uniformly bounded in $L^{\infty}(\mathfrak{m})$, we obtain strong convergence to $f_{\delta}$ in $L^{p}$ for $p \in[2, \infty)$ and $w^{*}$-convergence for $p=\infty$. It follows that $\int_{\{|h|>\delta\}}|h| d \mathfrak{m}=0$ and we can let $\delta \downarrow 0$ to get $h=0$.

To cover the cases $p=q \in[1,2)$, by interpolation we need only to consider $p=1$. Given $f \in L^{1}(\mathfrak{m})$ nonnegative, we can find $\varphi_{n} \in \mathscr{A}$ convergent to $\sqrt{f}$ in $L^{2}(\mathfrak{m})$. It follows that the functions $\varphi_{n}^{2}$ belong to $\mathscr{A}$ and converge to $f$ in $L^{1}(\mathfrak{m})$. In order to remove the sign assumption on $f$ we split in positive and negative part.

Finally, in the case $p<q$ we can use the density of bounded functions to reduce ourselves to the case of approximation of a bounded function $f \in L^{p} \cap L^{q}(\mathfrak{m})$ by functions in $\mathscr{A}$. Since $f$ can be approximated by equibounded functions $f_{n} \in \mathscr{A}$ in $L^{p}$ norm, we need only to use the fact that $f_{n} \rightarrow f$ also in $L^{q}$ norm.

Finally, a simple inspection of the proof shows that we can achieve the same density result with a countable subset of $\mathscr{A}$, since $\mathbb{V}$ is separable.

Remark 2.4. Under the weak "Feller" condition

$$
\mathscr{A} \text { is invariant under the action of } \mathrm{P}_{t} \text {, }
$$

our basic assumption that $\mathscr{A}$ is dense in $\mathbb{V}$ can be weakened to the assumption that $\mathscr{A}$ is dense in $L^{2}(\mathfrak{m})$; indeed, standard semigroup theory shows that an invariant subspace is dense in $\mathbb{V}$ if and only if it is invariant in $L^{2}(\mathfrak{m})$, see for instance [AGS11b, Lemma 4.9], but also Lemma 5.6 below.

\section{Derivations}

Since $\mathscr{A}$ might be regarded as an abstract space of test functions, we introduce derivations as linear operators acting on it, satisfying a Leibniz rule and a pointwise $\mathfrak{m}$-a.e. upper bound in terms of $\Gamma$ (even though for some results an integral bound would be sufficient).

Definition 3.1 (Derivation). A derivation is a linear operator $\boldsymbol{b}: \mathscr{A} \rightarrow L^{0}(\mathfrak{m}), f \mapsto d f(\boldsymbol{b})$, satisfying

$$
d(f g)(\boldsymbol{b})=f d g(\boldsymbol{b})+g d f(\boldsymbol{b}), \quad \mathfrak{m} \text {-a.e. in } X, \text { for every } f, g \in \mathscr{A}
$$

and

$$
|d f(\boldsymbol{b})| \leq g \sqrt{\Gamma(f)}, \quad \mathfrak{m} \text {-a.e. in } X, \text { for every } f \in \mathscr{A}
$$

for some $g \in L^{0}(\mathfrak{m})$. The smallest function $g$ with this property will be denoted by $|\boldsymbol{b}|$. For $p, q \in[1, \infty]$, we say that a derivation $\boldsymbol{b}$ is in $L^{p}+L^{q}$ if $|\boldsymbol{b}| \in L^{p}(\mathfrak{m})+L^{q}(\mathfrak{m})$.

Existence of the smallest function $g$ can easily be achieved using the fact that $L^{0}(\mathfrak{m})$ is a complete lattice, i.e. considering the supremum of $|d f(\boldsymbol{b})| \Gamma(f)^{-1 / 2}$ among all functions $f \in \mathscr{A}$ with $\Gamma(f) \neq 0$.

N. Gigli pointed out to us that linearity and the m-a.e. upper bound are sufficient to entail "locality" and thus Leibniz and chain rule, with a proof contained in the work in preparation [Gig14], akin to that of [AK00, Thm. 3.5]. Since our work focuses on the continuity equation and related Lagrangian flows, but not on the fine structure of the space of derivations, for the sake of simplicity, we have chosen to retain this slightly redundant definition and deduce only the validity of the chain rule. 
Proposition 3.2 (Chain rule for derivations). Let $\boldsymbol{b}$ be a derivation and let $\Phi: \mathbb{R}^{n} \rightarrow \mathbb{R}$ be a smooth function, with $\Phi(0)=0$. Then, for any $\boldsymbol{f}=\left(f_{1}, \ldots, f_{n}\right) \in \mathscr{A}^{n}$ there holds

$$
d \Phi(\boldsymbol{f})(\boldsymbol{b})=\sum_{i=1}^{n} \partial_{i} \Phi(\boldsymbol{f}) d f_{i}(\boldsymbol{b}), \quad \mathfrak{m} \text {-a.e. in } X .
$$

Proof. Since $\Phi(\boldsymbol{f}) \in \mathscr{A}, \boldsymbol{b}(\Phi(\boldsymbol{f}))$ is well defined. Arguing by induction and linearity, Leibniz rule entails that (3.1) holds when $\Phi$ is a polynomial in $n$ variables, with $\Phi(0)=0$. Since $\boldsymbol{f}$ is bounded, the thesis follows by approximating $\Phi$ with a sequence $\left(p_{k}\right)$ of polynomials, converging to $\Phi$, together with their derivatives, uniformly on compact sets.

Remark 3.3 (Derivations $u \boldsymbol{b}$ ). Let $\boldsymbol{b}$ be a derivation in $L^{q}$ for some $q \in[1, \infty]$ and let $u \in L^{r}(\mathfrak{m})$, with $q^{-1}+r^{-1} \leq 1$. Then, $f \mapsto u d f(\boldsymbol{b})$ defines a derivation $u \boldsymbol{b}$ in $L^{s^{\prime}}$, where $q^{-1}+r^{-1}+s^{-1}=1$. By linearity, similar remarks apply when $\boldsymbol{b}$ is a derivation in $L^{p}+L^{q}$

Example 3.4 (Gradient derivations). The main example is provided by derivations $\boldsymbol{b}_{g}$ induced by $g \in \mathbb{V}$, of the form

$$
f \in \mathscr{A} \mapsto(d f)\left(\boldsymbol{b}_{g}\right):=\Gamma(f, g) \in L^{1}(\mathfrak{m}) .
$$

These derivations belong to $L^{2}$, because $(2.6)$ yields $\left|\boldsymbol{b}_{g}\right| \leq \sqrt{\Gamma(g)}$. Since $\mathscr{A}$ is dense in $\mathbb{V}$, it is not difficult to show that equality holds.

By linearity, the $L^{\infty}$-module generated by this class of examples (i.e. finite sums $\sum_{i} \chi_{i} \boldsymbol{b}_{g_{i}}$ with $\chi_{i} \in L^{\infty}(\mathfrak{m})$ and $\left.g_{i} \in \mathbb{V}\right)$ still consists of derivations in $L^{2}$.

Definition 3.5 (Divergence). Let $p, q \in[1, \infty]$, assume that $\mathscr{A} \subset \mathbb{V}_{p^{\prime}} \cap \mathbb{V}_{q^{\prime}}$ and let $\boldsymbol{b}$ be a derivation in $L^{p}+L^{q}$. The distributional divergence $\operatorname{div} \boldsymbol{b}$ is the linear operator on $\mathscr{A}$ defined by

$$
\mathscr{A} \ni f \mapsto-\int d f(\boldsymbol{b}) d \mathfrak{m} .
$$

We say that $\operatorname{div} \boldsymbol{b} \in L^{p}(\mathfrak{m})+L^{q}(\mathfrak{m})$ if the distribution $\operatorname{div} \boldsymbol{b}$ is induced by $g \in L^{p}(\mathfrak{m})+L^{q}(\mathfrak{m})$, i.e.

$$
\int d f(\boldsymbol{b}) d \mathfrak{m}=-\int f g d \mathfrak{m}, \quad \text { for all } f \in \mathscr{A} .
$$

Analogously, we say that $\operatorname{div} \boldsymbol{b}^{-} \in L^{p}(\mathfrak{m})$ if there exists a nonnegative $g \in L^{p}(\mathfrak{m})$ such that

$$
\int d f(\boldsymbol{b}) d \mathfrak{m} \leq \int f g d \mathfrak{m}, \quad \text { for all } f \in \mathscr{A}, f \geq 0 .
$$

Notice that we impose the additional condition $\mathscr{A} \subset \mathbb{V}_{p^{\prime}} \cap \mathbb{V}_{q^{\prime}}$, to ensure integrability of $d f(\boldsymbol{b})$.

As we did for $|\boldsymbol{b}|$, we define $\operatorname{div} \boldsymbol{b}^{-}$as the smallest nonnegative function $g$ in $L^{p}(\mathfrak{m})$ for which the inequality above holds. Existence of the minimal $g$ follows by a simple convexity argument, because the class of admissible $g$ 's is convex and closed in $L^{p}(\mathfrak{m})$ (if $p=\infty$, one has to consider the $w^{*}$-topology).

Example 3.6 (Divergence of gradients). The distributional divergence of the "gradient" derivation $\boldsymbol{b}_{g}$ induced by $g \in \mathbb{V}$ as in (3.2) coincides with the Laplacian $\Delta g$, still understood in distributional terms. 
Although the definitions given above are sufficient for many purposes, the following extensions will be technically useful in Section 4.3 (and in Section 5 for the case $q \in[1, \infty)$ ).

Remark 3.7 (Derivations in $L^{2}+L^{\infty}$ extend to $\mathbb{V}$ ). When a derivation $\boldsymbol{b}$ belongs to $L^{2}+L^{\infty}$, we can use the density of $\mathscr{A}$ in $\mathbb{V}$ to extend uniquely $\boldsymbol{b}$ to a derivation, still denoted by $\boldsymbol{b}$, defined on $\mathbb{V}$, with values in the space $L^{1}(\mathfrak{m})+L^{2}(\mathfrak{m})$ and continuous. For all $u \in \mathbb{V}$, it still satisfies

$$
|d u(\boldsymbol{b})| \leq|\boldsymbol{b}| \sqrt{\Gamma(u)} \quad \mathfrak{m} \text {-a.e. in } X .
$$

A similar remark holds for derivations belonging to $L^{q}+L^{\infty}$, for some $q \in[1, \infty)$, if $\mathscr{A}$ is assumed to be dense in $\mathbb{V}_{r}$, for some $r \in[1, \infty)$ with $q^{-1}+r^{-1} \leq 1$. The extension is then a continuous linear operator $\boldsymbol{b}$ mapping $\mathbb{V}_{r}$ into $L^{s^{\prime}}(\mathfrak{m})+L^{2}(\mathfrak{m})$, where $q^{-1}+r^{-1}+s^{-1}=1$.

By a similar density argument as above, any derivation $\boldsymbol{b}$ could be extended uniquely to a derivation defined on $\mathbb{V}$, with values in the space $L^{0}(\mathfrak{m})$. However, such an extension is not useful when dealing with integral functionals defined initially on $\mathscr{A}$, e.g. that of divergence or weak solutions to the continuity equation, because these are not continuous with respect to the topology of $L^{0}(\mathfrak{m})$. Therefore, we avoid in what follows to consider such an extension, except for the case in the remark above.

We conclude this section noticing that if $\boldsymbol{b}$ is a derivation $L^{2}+L^{\infty}$, with $\operatorname{div} \boldsymbol{b} \in L^{2}(\mathfrak{m})+$ $L^{\infty}(\mathfrak{m})$, the following integration by parts formula can be proved by approximation with functions in $\mathscr{A}$ :

$$
\int d u(\boldsymbol{b}) f d \mathfrak{m}=-\int d f(\boldsymbol{b}) u d \mathfrak{m}+\int u f \operatorname{div} \boldsymbol{b} d \mathfrak{m} \quad \forall f \in \mathscr{A}, \forall u \in \mathbb{V} .
$$

\section{Existence of solutions to the continuity equation}

Let $I=(0, T)$ with $T \in(0, \infty)$. In this section we prove existence of weak solutions to the continuity equation

$$
\frac{d}{d t} u_{t}+\operatorname{div}\left(u_{t} \boldsymbol{b}_{t}\right)=w_{t} \quad \text { in } I \times X
$$

under suitable growth assumptions on $\boldsymbol{b}_{t}$ and its divergence.

Remark 4.1. Starting from this section, we always assume that $\mathscr{A}$ is contained in $\mathbb{V}_{\infty}$, i.e. $\Gamma(f) \in L^{\infty}(\mathfrak{m})$ for every $f \in \mathscr{A}$. We are motivated by the examples and by the clarity that we gain in the exposition, although some variants of our results could be slightly reformulated and proved without this assumption.

Before we address the definition of (4.1), let us remark that a Borel family of derivations $\boldsymbol{b}=\left(\boldsymbol{b}_{t}\right)_{t \in I}$ is by definition a map $t \mapsto \boldsymbol{b}_{t}$, taking values in the space of derivations on $X$, such that there exists a Borel function $g: I \times X \mapsto[0, \infty)$ satisfying

$$
\left|\boldsymbol{b}_{t}\right| \leq g(t, \cdot) \mathfrak{m} \text {-a.e. in } X \text {, for a.e. } t \in I \text {. }
$$

As in the autonomous case we denote by $|\boldsymbol{b}|$ the smallest function $g$ (in the $\mathcal{L}^{1} \otimes \mathfrak{m}$-a.e. sense) with this property. We say that Borel family of derivations $\left(\boldsymbol{b}_{t}\right)_{t \in I}$ belongs to $L_{t}^{r}\left(L_{x}^{p}+L_{x}^{q}\right)$ if $|\boldsymbol{b}| \in L_{t}^{r}\left(L_{x}^{p}+L_{x}^{q}\right)$. 
Definition 4.2 (Weak solutions to the continuity equation with initial condition $\bar{u}$ ). Let $p, q \in[1, \infty], \bar{u} \in L^{p} \cap L^{q}(\mathfrak{m})$, let $\left(\boldsymbol{b}_{t}\right)_{t \in I}$ be a Borel family of derivations in $L_{t}^{1}\left(L_{x}^{p^{\prime}}+L_{x}^{q^{\prime}}\right)$ and let $w \in L_{t}^{1}\left(L_{x}^{p^{\prime}}+L_{x}^{q^{\prime}}\right)$. We say that $u \in L_{t}^{\infty}\left(L_{x}^{p} \cap L_{x}^{q}\right)$ solves (4.1) with the initial condition $u_{0}=\bar{u}$ in the weak sense if

$$
\int_{0}^{T} \int\left[-\psi^{\prime} \varphi-\psi d \varphi\left(\boldsymbol{b}_{t}\right)-w_{t}\right] u_{t} d \mathfrak{m} d t=\psi(0) \int \varphi \bar{u} d \mathfrak{m}
$$

for all $\varphi \in \mathscr{A}$ and all $\psi \in C^{1}([0, T])$ with $\psi(T)=0$.

Notice that, without the assumption $\mathscr{A} \subset \mathbb{V}_{\infty}$, one could define e.g. weak solutions $u \in L_{t}^{\infty}\left(L_{x}^{2}\right)$ to the equation associated to $\boldsymbol{b}$ in $L_{t}^{1}\left(L_{x}^{\infty}\right)$.

In order to prove the mass-conservation property of solutions to the continuity equation we assume the existence of $\left(f_{n}\right) \subset \mathscr{A}$ satisfying

$$
0 \leq f_{n} \leq 1, f_{n} \uparrow 1 \mathfrak{m} \text {-a.e. in } X, \sqrt{\Gamma\left(f_{n}\right)} \rightarrow 0 \text { weakly-* in } L^{\infty}(\mathfrak{m}) .
$$

The following theorem is our main result about existence: we address the case $w=0$ only, the general case following from a Duhamel's principle that we do not pursue here.

Theorem 4.3 (Existence of weak solutions in $L_{t}^{\infty}\left(L_{x}^{1} \cap L_{x}^{2}\right)$ ). Assume that $\mathscr{A} \subset \mathbb{V}_{\infty}$, let $\bar{u} \in L^{1} \cap L^{r}(\mathfrak{m})$ for some $r \in[2, \infty]$ and let $\boldsymbol{b}=\left(\boldsymbol{b}_{t}\right)_{t \in I}$ be a Borel family of derivations with $|\boldsymbol{b}| \in L_{t}^{1}\left(L_{x}^{2}+L_{x}^{\infty}\right)$, div $\boldsymbol{b} \in L_{t}^{1}\left(L_{x}^{2}+L_{x}^{\infty}\right)$, and $\operatorname{div} \boldsymbol{b}^{-} \in L_{t}^{1}\left(L_{x}^{\infty}\right)$.

Then, there exists a weakly continuous in $[0, T)$ (in duality with $\mathscr{A}$ ) solution $u \in L_{t}^{\infty}\left(L_{x}^{1} \cap L_{x}^{r}\right)$ of (4.1) according to Definition 4.2 with $u_{0}=\bar{u}$ and $w_{t}=0$. Furthermore, if $\bar{u} \geq 0$, we can build a solution $u$ in such a way that $u_{t} \geq 0$ for all $t \in I$. Finally, if (4.3) holds, then

$$
\int u_{t} d \mathfrak{m}=\int \bar{u} d \mathfrak{m} \quad \forall t \in[0, T) .
$$

To prove existence of a solution $u$ to (4.1) with $w_{t}=0$, we rely on a suitable approximation of the equation. Following a classical strategy, we approximate the original equation by adding a diffusion term, i.e. we solve, still in the weak sense of duality with test functions $\psi(t) \varphi(x)$,

$$
\partial_{t} u_{t}+\operatorname{div}\left(u_{t} \boldsymbol{b}_{t}\right)=\sigma \Delta u_{t}
$$

where $\sigma>0$. By Hilbert space techniques, we show existence of a solution with some extra regularity, namely $u \in L^{2}(I ; \mathbb{V})$. We use this extra regularity to derive a priori estimates and then we take weak limits as $\sigma \downarrow 0$.

\subsection{Auxiliary Hilbert spaces}

In all what follows, we consider the Gelfand triple

$$
\mathbb{V} \subset L^{2}(\mathfrak{m})=\left(L^{2}(\mathfrak{m})\right)^{*} \subset \mathbb{V}^{\prime},
$$

i.e. we regard $\mathbb{V}$ as a dense subspace in $\mathbb{V}^{\prime}$ (proper if $\mathbb{V} \neq L^{2}(\mathfrak{m})$ ) by means of

$$
\phi \mapsto\left(\phi^{*}: f \mapsto \int f \phi d \mathfrak{m}\right)
$$


Notice that this is different from the identification $\mathbb{V} \sim \mathbb{V}^{\prime}$ provided by the Riesz-Fischer theorem applied to the Hilbert space $\mathbb{V}$ (which has been applied to $L^{2}(\mathfrak{m})$ instead).

Given a vector space $F$, we introduce a space of $F$-valued test functions on $I$, namely

$$
\Phi_{F}:=\operatorname{span}\left\{\psi \cdot \phi: \psi \in C^{1}([0, T]), \psi(T)=0, \phi \in F\right\} .
$$

We notice that, for every $\varphi \in \Phi_{F}$, the function $t \mapsto \varphi_{t}$ is Lipschitz and continuously differentiable from $I$ to $F$, and there exists $\varphi_{0}=\lim _{t \downarrow 0} \varphi_{t}$ in $F$ (while $\lim _{t \uparrow T} \varphi_{t}=0$ in $F$ by construction).

Assuming that $F$ is a separable Hilbert space, starting from $\Phi_{F}$ one can consider completions with respect to different norms. The classical space

$$
L^{2}(I ; F), \quad\langle\varphi, u\rangle_{L^{2}(F)}=\int_{I}\left\langle\phi_{t}, u_{t}\right\rangle_{F} d t
$$

is indeed the closure of $\Phi_{F}$ with respect to the norm induced by the scalar product above. Similarly, the space $H^{1}(I ; F)$ is obtained by completing $\Phi_{F}$ with respect to the norm

$$
\langle\varphi, u\rangle_{H^{1}(F)}=\int_{I}\left\langle\phi_{t}, u_{t}\right\rangle_{F}+\left\langle\frac{d}{d t} \phi_{t}, \frac{d}{d t} u_{t}\right\rangle_{F} d t
$$

Arguing by mollification as in the case $F=\mathbb{R}^{n}$, it is not difficult to prove that $H^{1}(I ; F)=$ $W^{1,2}(I ; F)$, where the latter space is defined as the subspace of functions $u \in L^{2}(I ; F)$ such that there exists $g \in L^{2}(I ; F)$, which represent the distributional derivative of $u$, i.e.

$$
\int_{I}\left\langle u_{t}, \frac{d}{d t} \varphi_{t}\right\rangle_{F} d t=-\int_{0}^{T}\left\langle g_{t}, \varphi_{t}\right\rangle_{F} d t, \quad \text { for every } \varphi \in \Phi_{F} \text { with } \varphi_{0}=0
$$

\subsection{Existence under additional ellipticity}

We address now the existence of some $u \in L^{2}(I ; \mathbb{V})$ that solves the following weak formulation of (4.5) with the initial condition $u_{0}=\bar{u}$ :

$$
\int_{0}^{T} \int\left[-\partial_{t} \varphi_{t}-d \varphi_{t}\left(\boldsymbol{b}_{t}\right)\right] u_{t}+\sigma \Gamma\left(\varphi_{t}, u_{t}\right) d \mathfrak{m} d t=\int \varphi_{0} \bar{u} d \mathfrak{m} \quad \forall \varphi \in \Phi_{\mathscr{A}}
$$

We still assume that $\mathscr{A} \subset \mathbb{V}_{\infty}$, and that $\sigma \in(0,1 / 2],|\boldsymbol{b}| \in L_{t}^{\infty}\left(L_{x}^{2}+L_{x}^{\infty}\right)$, div $\boldsymbol{b}^{-} \in L_{t}^{\infty}\left(L_{x}^{\infty}\right)$, $\bar{u} \in L^{2}(\mathfrak{m})$. Notice that the assumptions on $|\boldsymbol{b}|$ and $\operatorname{div} \boldsymbol{b}^{-}$are stronger than that in Theorem 4.3 , but only with respect to integrability in time.

We obtain, together with existence, the a priori estimate:

$$
\left\|e^{-\lambda t} u\right\|_{L^{2}(I ; \mathbb{V})} \leq \frac{\|\bar{u}\|_{2}}{\sigma} \quad \text { with } \quad \lambda:=\frac{1}{2}\left\|\operatorname{div} \boldsymbol{b}^{-}\right\|_{\infty}+\sigma .
$$

To this aim, we change variables setting $h_{t}=e^{-\lambda t} u_{t}$ and we pass to this equivalent weak formulation

$$
\int_{0}^{T} \int\left[-\partial_{t} \varphi_{t}+\lambda-d \varphi_{t}\left(\boldsymbol{b}_{t}\right)\right] h_{t}+\sigma \Gamma\left(\varphi_{t}, h_{t}\right) d \mathfrak{m} d t=\int \varphi_{0} \bar{u} d \mathfrak{m} \quad \forall \varphi \in \Phi_{\mathscr{A}}
$$

From now on we shall use the notation $\widetilde{\mathfrak{m}}$ for the product measure $\mathscr{L}^{1} \otimes \mathfrak{m}$ in $I \times X$. Existence of $h$ is a consequence of J.-L. Lions' extension of Lax-Milgram Theorem, whose 
statement is recalled below (see [Sho97, Thm. III.2.1, Corollary III.2.3]) applied with $H=$ $L^{2}(I ; \mathbb{V}), V=\Phi_{\mathscr{A}}$ endowed with the norm

$$
\begin{gathered}
\|\varphi\|_{V}^{2}=\|\varphi\|_{L^{2}(I ; \mathbb{V})}^{2}+\left\|\varphi_{0}\right\|_{2}^{2}, \\
B(\varphi, h)=\int\left[-\partial_{t} \varphi+\lambda \varphi-d \varphi(\boldsymbol{b})\right] h+\sigma \Gamma(\varphi, h) d \widetilde{\mathfrak{m}}, \quad \ell(\varphi)=\int \varphi_{0} \bar{u} d \mathfrak{m} .
\end{gathered}
$$

Theorem 4.4 (Lions). Let $V, H$ be respectively a normed and a Hilbert space, with $V$ continuously embedded in $H$, with $\|v\|_{H} \leq\|v\|_{V}$ for all $v \in V$, and let $B: V \times H \rightarrow \mathbb{R}$ be bilinear, with $B(v, \cdot)$ continuous for all $v \in V$. If $B$ is coercive, namely there exists $c>0$ satisfying $B(v, v) \geq c\|v\|_{V}^{2}$ for all $v \in V$, then for all $\ell \in V^{\prime}$ there exists $h \in H$ such that $B(\cdot, h)=\ell$ and

$$
\|h\|_{H} \leq \frac{\|\ell\|_{V^{\prime}}}{c}
$$

Let us start by checking coercivity (here the change of variables we did and the choice of $\lambda$ play a role):

$$
\begin{aligned}
\int[\lambda \varphi-d \varphi(\boldsymbol{b})] \varphi d \widetilde{\mathfrak{m}} & =\lambda\|\varphi\|_{1,2}^{2}-\frac{1}{2} \int d \varphi^{2}(\boldsymbol{b}) d \widetilde{\mathfrak{m}} \\
& \geq \lambda\|\varphi\|_{1,2}^{2}-\frac{1}{2} \int \varphi^{2} \operatorname{div} \boldsymbol{b}^{-} d \widetilde{\mathfrak{m}} \\
& \geq\left(\lambda-\frac{1}{2}\left\|\operatorname{div} \boldsymbol{b}^{-}\right\|_{\infty}\right)\|\varphi\|_{1,2}^{2}=\sigma\|\varphi\|_{1,2}^{2} .
\end{aligned}
$$

Since $\varphi \in V=\Phi_{\mathscr{A}}$, it holds $\partial_{t} \varphi_{t}^{2}=2 \varphi_{t} \partial_{t} \varphi_{t}$ and $\int-2 \varphi_{t} \partial \varphi d \widetilde{\mathfrak{m}}=\int \varphi_{0}^{2} d \mathfrak{m}$. Hence, inequality (4.11) entails that

$$
\int\left[-\partial_{t} \varphi+\lambda \varphi-d \varphi(\boldsymbol{b})\right] \varphi+\sigma \Gamma(\varphi) d \widetilde{\mathfrak{m}} \geq \frac{1}{2} \int \varphi_{0}^{2} d \mathfrak{m}+\sigma\|\varphi\|_{1,2}^{2}+\sigma\|\sqrt{\Gamma(\varphi)}\|_{1,2}^{2},
$$

Since $\sigma \leq 1 / 2$, it follows from these two inequalities that

$$
B(\varphi, \varphi) \geq \sigma\|\varphi\|_{V}^{2}
$$

To prove continuity, let $\varphi \in V$. The linear functional $h \mapsto B(\varphi, h)$ is $L^{2}(I ; \mathbb{V})$-continuous for all $\phi \in V$, since we can estimate $|B(\varphi, h)|$ from above with

$$
\|h\|_{L^{2}(I ; \mathbb{V})}\left[\left\|\partial_{t} \varphi\right\|_{L_{t}^{1}\left(L_{x}^{2}\right)}+\lambda\|\varphi\|_{L_{t}^{1}\left(L_{x}^{2}\right)}+\|\boldsymbol{b}\|_{L_{t}^{2}\left(L_{x}^{2}+L_{x}^{\infty}\right)}\|\sqrt{\Gamma(\varphi)}\|_{L_{t}^{\infty}\left(L_{x}^{2} \cap L_{x}^{\infty}\right)}+\sigma\|\sqrt{\Gamma(\varphi)}\|_{L_{t}^{1}\left(L_{x}^{2}\right)}\right] .
$$

The functional $\ell$ satisfies $\|\ell\|_{V^{\prime}} \leq\|\bar{u}\|_{2}$, immediately from the definition of $\|\cdot\|_{V}$ in (4.9), concluding the verification of the assumptions of Theorem 4.4.

Finally, (4.7) follows at once from (4.10) and (4.12), taking into account that $\|\ell\|_{V^{\prime}} \leq\|\bar{u}\|_{2}$.

\subsection{A priori estimates}

In this section we still consider weak solutions to

$$
\int_{0}^{T} \int-\left[\partial_{t} \varphi_{t}+d \varphi_{t}\left(\boldsymbol{b}_{t}\right)\right] u_{t}+\sigma \Gamma\left(\varphi_{t}, u_{t}\right) d \mathfrak{m} d t=\int \varphi_{0} \bar{u} d \mathfrak{m} \quad \forall \varphi \in \Phi_{\mathscr{A}}
$$

obtained in the previous section. In order to state pointwise in time $L^{r}$ estimates in space, we use the following remark. 
Remark 4.5 (Equivalent formulation). Assuming $\mathscr{A} \subset \mathbb{V}_{\infty}, u \in L^{2}(I ; \mathbb{V})$ and $|\boldsymbol{b}| \in L_{t}^{1}\left(L_{x}^{2}+\right.$ $\left.L_{x}^{\infty}\right)$, an equivalent formulation of (4.13), in terms of absolute continuity and pointwise derivatives w.r.t. time, is the following: we are requiring that, for every $f \in \mathscr{A}, t \mapsto \int f u_{t} d \mathfrak{m}$ is absolutely continuous in $I$ and that its a.e. derivative in $I$ is $\int\left(d f\left(\boldsymbol{b}_{t}\right) u_{t}+\sigma \Gamma\left(f, u_{t}\right)\right) d \mathfrak{m}$. In addition, the Cauchy initial condition is encoded by

$$
\lim _{t \downarrow 0} \int f u_{t} d \mathfrak{m}=\int f \bar{u} d \mathfrak{m}, \quad \text { for every } f \in \mathscr{A}
$$

(notice also that $\bar{u}$ is uniquely determined by (4.14), thanks to the density of $\mathscr{A}$ in $L^{2}(\mathfrak{m})$ ).

Indeed, it is clear that the definition above implies the formula for the distributional derivative, because for absolutely continuous functions the two concepts coincide; the converse can be obtained using the set $\mathscr{D}$ of Lemma 2.3 to redefine $u_{t}$ is a negligible set of times in order to get a weakly continuous representative in the duality with $\mathscr{A}$, see [AGS05, Lemma 8.1.2] for details.

We prove, by a suitable approximation, the following result:

Theorem 4.6. Assume that $\mathscr{A} \subset \mathbb{V}_{\infty},|\boldsymbol{b}| \in L_{t}^{\infty}\left(L_{x}^{2}+L_{x}^{\infty}\right)$, $\operatorname{div} \boldsymbol{b} \in L_{t}^{\infty}\left(L_{x}^{2}+L_{x}^{\infty}\right)$, $\operatorname{div} \boldsymbol{b}^{-} \in$ $L_{t}^{\infty}\left(L_{x}^{\infty}\right)$, and that the initial condition $\bar{u}$ belongs to $L^{p} \cap L^{q}(\mathfrak{m})$, with $1 \leq p \leq 2 \leq q \leq \infty$. Then there exists a weakly continuous (in duality with $\mathscr{A}$ ) solution

$$
u \in L_{t}^{\infty}\left(L_{x}^{p} \cap L_{x}^{q}\right) \cap L^{2}(I ; \mathbb{V})
$$

to (4.13) satisfying:

$$
\sup _{(0, T)}\left\|u_{t}^{ \pm}\right\|_{r} \leq\left\|\bar{u}^{ \pm}\right\|_{r} \exp \left(\left(1-\frac{1}{r}\right)\left\|\operatorname{div} \boldsymbol{b}^{-}\right\|_{1, \infty}\right)
$$

for every $r \in[p, q]$. In particular, if $\bar{u} \geq 0$, then $u_{t} \geq 0$ for all $t \in(0, T)$.

At this stage, it is technically useful to introduce another formulation of the continuity equation, suitable for $\mathbb{V}$-valued solutions $u$, with the derivation acting on $u$.

Remark 4.7 (Transport weak formulation). Using (3.3) we obtain an equivalent weak formulation of (4.13), namely

$$
\int_{0}^{T} \int-u_{t} \partial_{t} \varphi_{t}+d u_{t}\left(\boldsymbol{b}_{t}\right) \varphi_{t}+u_{t} \varphi_{t} \operatorname{div} \boldsymbol{b}_{t}+\sigma \Gamma\left(\varphi_{t}, u_{t}\right) d \mathfrak{m} d t=\int \varphi_{0} \bar{u} d \mathfrak{m} \quad \forall \varphi \in \Phi_{\mathscr{A}}
$$

Remark 4.8 (Basic formal identity). Before we address the proof of the a priori estimates, let us remark that these, and uniqueness as well, strongly rely on the formal identity

$$
\frac{d}{d t} \int \beta\left(u_{t}\right) d \mathfrak{m}=-\int\left[\beta^{\prime}\left(u_{t}\right) u_{t}-\beta\left(u_{t}\right)\right] \operatorname{div} \boldsymbol{b}_{t} d \mathfrak{m},
$$

which comes from chain rule in (4.2) and the formal identity $\int \operatorname{div}\left(\beta\left(u_{t}\right) \boldsymbol{b}_{t}\right)=0$. To establish existence, however, this computation is made rigorous by approximating the PDE (by vanishing viscosity, or other approximations), while to obtain uniqueness in Section 5 we approximate $u$. In both cases technical assumptions on $\boldsymbol{b}$ will be needed. 
A natural choice in (4.17) is a convex "entropy" function $\beta: \mathbb{R} \rightarrow \mathbb{R}$ with $\beta(0)=0$. In order to give a meaning to the identity (4.17) also when $\beta$ is not $C^{1}\left(z \mapsto z^{+}\right.$will be a typical choice of $\beta$ ) we define

$$
\mathcal{L}_{\beta}(z):= \begin{cases}z \beta_{+}^{\prime}(z)-\beta(z) & \text { if } z \geq 0 \\ z \beta_{-}^{\prime}(z)-\beta(z) & \text { if } z \leq 0 .\end{cases}
$$

The function $\mathcal{L}_{\beta}$ is the Legendre transform of $\beta$. Notice that the convexity of $\beta$ and the condition $\beta(0)=0$ give that $\mathcal{L}_{\beta}$ is nonnegative; for instance, if $z \geq 0$, there holds

$$
\beta(0)=0 \geq \beta(z)-z \beta_{-}^{\prime}(z) \geq \beta(z)-z \beta_{+}^{\prime}(z) .
$$

The argument for $z \leq 0$ follows from $\mathcal{L}_{\tilde{\beta}}(-z)=\mathcal{L}_{\beta}(z)$, where $\tilde{\beta}(z)=\beta(-z)$. It is also easy to check, with a similar argument, that $\mathcal{L}_{\beta}$ is nondecreasing $\left(\left(\mathcal{L}_{\beta}\right)^{\prime}=z \beta^{\prime \prime}\right.$ in the smooth case).

In order to approximate $\beta$ with functions with linear growth in $\mathbb{R}$, we will consider the approximations

$$
\beta_{n}(z):= \begin{cases}\beta(-n)+\beta_{-}^{\prime}(-n)(z+n) & \text { if } z<-n ; \\ \beta(z) & \text { if }-n \leq z \leq n \\ \beta(n)+\beta_{+}^{\prime}(n)(z-n) & \text { if } z>n,\end{cases}
$$

that satisfy $\mathcal{L}_{\beta_{n}}(z)=\mathcal{L}_{\beta}(-n \vee z \wedge n)$, so that $\mathcal{L}_{\beta_{n}} \uparrow \mathcal{L}_{\beta}$ as $n \rightarrow \infty$. On the other hand, in order to pass from smooth to nonsmooth $\beta$ 's, we will also need the following property, whose proof is elementary and motivates our precise definition of $\mathcal{L}_{\beta}$ in (4.18):

$\limsup _{i \rightarrow \infty} \mathcal{L}_{\beta_{i}} \leq \mathcal{L}_{\beta} \quad$ whenever $\beta_{i}$ are convex, $\beta_{i} \rightarrow \beta$ uniformly on compact sets.

Proof of Theorem 4.6. By Remark 4.5 we can assume with no loss of generality that $t \mapsto u_{t}$ is weakly continuous in $[0, T)$, in the duality with $\mathscr{A}$.

We assume first that a weak solution $u$ satisfies the strong continuity property

$$
\lim _{t \downarrow 0} u_{t}=\bar{u} \quad \text { in } L^{2}(\mathfrak{m}) .
$$

We shall remove this assumption at the end of the proof.

We claim that for any convex function $\beta: \mathbb{R} \rightarrow[0, \infty)$ satisfying $\beta(0)=0$ and $\beta^{\prime}(z) / z$ bounded on $\mathbb{R}$, the inequality

$$
\frac{d}{d t} \int \beta\left(u_{t}\right) d \mathfrak{m} \leq \int \mathcal{L}_{\beta}\left(u_{t}\right) \operatorname{div} \boldsymbol{b}_{t}^{-} d \mathfrak{m}
$$

holds in the sense of distributions in $(0, T)$. The assumption on the behaviour of $\beta$ near to the origin is needed to ensure that both $\beta(u)$ and $\mathcal{L}_{\beta}(u)$ belong to $L_{t}^{2}\left(L_{x}^{1}\right)$, since at present we only know that $u \in L_{t}^{2}\left(L_{x}^{2}\right)$. By approximation, taking (4.19) and (4.20) into account, we can assume with no loss of generality that $\beta \in C^{1}$ with bounded derivative.

In the proof of (4.22), motivated by the necessity to get strong differentiability w.r.t. time, we shall use the regularization $u_{t}^{s}:=\mathrm{P}_{s} u_{t}$ and the following elementary remark ([Sho97, Prop. III.1.1]). 
Remark 4.9. Let $X$ be a Banach space, let $f, g \in L^{1}((0, T) ; X)$ satisfy $\partial_{t} f=g$ in the weak sense, namely

$$
-\int_{0}^{T} \psi^{\prime}(t) \int \phi(f) d \mathfrak{m} d t=\int_{0}^{T} \psi(t) \int \phi(g) d \mathfrak{m} d t
$$

for every $\psi \in C_{c}^{1}(0, T), \phi \in \mathcal{D} \subset X^{*}$, dense w.r.t. the $\sigma\left(X^{*}, X\right)$-topology. Then, $f$ is absolutely continuous from $I$ to $X$ and strongly differentiable a.e. in $I$, with derivative equal to $g$.

Notice that $X$ may not have the Radon-Nikodym property so that it might be the case that not all absolutely continuous maps with values in $X$ are strongly differentiable a.e. in their domain. Indeed, we are going to apply it with $X=L^{1}(\mathfrak{m})+L^{2}(\mathfrak{m})$, so that $X^{*}=L^{2} \cap L^{\infty}(\mathfrak{m})$, and $\mathcal{D}=\mathscr{A}$.

It is immediate to check, replacing $\varphi$ in (4.16) by $\mathrm{P}_{s} \varphi$ and using (3.3), that for any $s>0$ the function $t \mapsto u_{t}^{s}$ solves

$$
\frac{d}{d t} u_{t}^{s}+\operatorname{div}\left(\boldsymbol{b}_{t} u_{t}^{s}\right)=\sigma \Delta u_{t}^{s}+\mathscr{C}_{t}^{s}
$$

in the weak sense of duality with $\mathscr{A}$, where $\mathscr{C}_{t}^{s}$ is the commutator between semigroup and divergence, namely

$$
\mathscr{C}_{t}^{s}:=\operatorname{div}\left(\boldsymbol{b}_{t} u_{t}^{s}\right)-\mathrm{P}_{s}\left(\operatorname{div}\left(\boldsymbol{b}_{t} u_{t}\right)\right) .
$$

Therefore, using (3.3) once more and expanding

$$
\mathscr{C}_{t}^{s}=u_{t}^{s} \operatorname{div} \boldsymbol{b}_{t}+d u_{t}^{s}\left(\boldsymbol{b}_{t}\right)-\mathrm{P}_{s}\left(u_{t} \operatorname{div} \boldsymbol{b}_{t}\right)-\mathrm{P}_{s}\left(d u_{t}\left(\boldsymbol{b}_{t}\right)\right)
$$

we may use the assumption $\operatorname{div} \boldsymbol{b} \in L_{t}^{\infty}\left(L_{x}^{2}+L_{x}^{\infty}\right)$ and the continuity of derivations to obtain that $\mathscr{C}_{t}^{s} \rightarrow 0$ strongly in $L_{t}^{2}\left(L_{x}^{1}+L_{x}^{2}\right)$ as $s \downarrow 0$. Similarly, expanding $\operatorname{div}\left(\boldsymbol{b}_{t} u_{t}^{s}\right)=u_{t}^{s} \operatorname{div} \boldsymbol{b}_{t}+$ $d u_{t}^{s}\left(\boldsymbol{b}_{t}\right)$ and using the regularization estimate (2.13) to estimate the Laplacian term in the derivative of $u_{t}$ we obtain $\frac{d}{d t} u_{t}^{s} \in L_{t}^{2}\left(L_{x}^{1}+L_{x}^{2}\right)$ in the weak sense of duality with $\mathscr{A}$, therefore $t \mapsto u_{t}^{s}$ is strongly $\left(L^{1}+L^{2}\right)$-differentiable a.e. in $(0, T)$, and absolutely continuous.

Since $\beta$ is convex, we can start from the inequality

$$
\int \beta\left(u_{t}^{s}\right) d \mathfrak{m}-\int \beta\left(u_{t^{\prime}}^{s}\right) d \mathfrak{m} \leq \int \beta^{\prime}\left(u_{t}^{s}\right)\left(u_{t}^{s}-u_{t^{\prime}}^{s}\right) d \mathfrak{m}
$$

and we can use the uniform boundedness of $\beta^{\prime}(z)$ and of $\beta^{\prime}(z) / z$ to obtain that $\beta^{\prime}\left(u_{t}^{s}\right) \in$ $L_{t}^{2}\left(L_{x}^{2} \cap L_{x}^{\infty}\right)$, hence

$$
\int \beta\left(u_{t}^{s}\right) d \mathfrak{m}-\int \beta\left(u_{t^{\prime}}^{s}\right) d \mathfrak{m} \leq g(t)\left|\int_{t^{\prime}}^{t}\left\|\frac{d}{d r} u_{r}^{s}\right\|_{L^{1}+L^{2}} d r\right|
$$

with $g(t)=\left\|\beta^{\prime}\left(u_{t}^{s}\right)\right\|_{L^{2} \cap L^{\infty}} \in L^{2}(0, T)$. Since (again by the convexity of $\beta$ ) $t \mapsto \int \beta\left(u_{t}^{s}\right) d \mathfrak{m}$ is lower semicontinuous, we can apply a calculus lemma [AGS11a, Lemma 2.9] to obtain that $t \mapsto \int \beta\left(u_{t}^{s}\right) d \mathfrak{m}$ is absolutely continuous in $(0, T)$ and that

$$
\frac{d}{d t} \int \beta\left(u_{t}^{s}\right) d \mathfrak{m}=\int \beta^{\prime}\left(u_{t}^{s}\right)\left[-\operatorname{div}\left(\boldsymbol{b}_{t} u_{t}^{s}\right)+\sigma \Delta u_{t}^{s}+\mathscr{C}_{t}^{s}\right] d \mathfrak{m}
$$

for a.e. $t \in(0, T)$. 
Since $\beta\left(u_{t}^{s}\right) \in \mathbb{V}$ we get $\int \beta^{\prime}\left(u_{t}^{s}\right) \Delta u_{t}^{s} d \mathfrak{m}=-\int \beta^{\prime \prime}\left(u_{t}^{s}\right) \Gamma\left(u_{t}^{s}\right) d \mathfrak{m} \leq 0$, hence we may disregard this term. Using twice the chain rule and $\operatorname{div} \boldsymbol{b} \in L_{t}^{2}\left(L_{x}^{2}+L_{x}^{\infty}\right), \mathcal{L}_{\beta}(u) \in L_{t}^{2}\left(L_{x}^{1}\right)$ gives

$$
\begin{aligned}
\frac{d}{d t} \int \beta\left(u_{t}^{s}\right) d \mathfrak{m} & \leq-\int \beta^{\prime}\left(u_{t}^{s}\right) u_{t}^{s} \operatorname{div} \boldsymbol{b}_{t}+d \beta\left(u_{t}^{s}\right)\left(\boldsymbol{b}_{t}\right) d \mathfrak{m}+\int \beta^{\prime}\left(u_{t}^{s}\right) \mathscr{C}_{t}^{s} d \mathfrak{m} \\
& =-\int\left(\beta^{\prime}\left(u_{t}^{s}\right) u_{t}^{s}-\beta\left(u_{t}^{s}\right)\right) \operatorname{div} \boldsymbol{b}_{t} d \mathfrak{m}+\int \beta^{\prime}\left(u_{t}^{s}\right) \mathscr{C}_{t}^{s} d \mathfrak{m} \\
& \leq \int\left(\beta^{\prime}\left(u_{t}^{s}\right) u_{t}^{s}-\beta\left(u_{t}^{s}\right)\right) \operatorname{div} \boldsymbol{b}_{t}^{-} d \mathfrak{m}+\int \beta^{\prime}\left(u_{t}^{s}\right) \mathscr{C}_{t}^{s} d \mathfrak{m} .
\end{aligned}
$$

Eventually, since $\beta^{\prime}\left(u_{t}^{s}\right)$ are bounded in $L_{t}^{2}\left(L_{x}^{2} \cap L_{x}^{\infty}\right)$, uniformly w.r.t. $s$, we let $s \downarrow 0$ to obtain (4.22).

Proof of (4.15). Let $r \in[p, q]$, let $\beta(z)=\left(z^{+}\right)^{r}$ and notice that $\mathcal{L}_{\beta}(z)=(r-1) \beta(z)$. We cannot apply directly (4.22) to $\beta$, because $\beta^{\prime}(z) / z$ is unbounded near 0 . If $r<2$, we let

$$
\beta_{n}(z):= \begin{cases}\frac{\left(z^{+}\right)^{2}}{2 \epsilon^{2-r}} & \text { if } z \leq \epsilon ; \\ \left(z^{+}\right)^{r}-\frac{\epsilon^{r}}{2} & \text { if } z \geq \epsilon,\end{cases}
$$

where $\varepsilon=1 / n$, so that $\beta_{n}$ are convex, $\beta_{n}^{\prime}(z) / z$ is bounded, $\mathcal{L}_{\beta_{n}} \leq \beta_{n}$ and $\beta_{n} \rightarrow \beta$ as $n \rightarrow \infty$.

If $r \geq 2$, we use the approximations $\beta_{n}$ in (4.19), that satisfy $\mathcal{L}_{\beta_{n}}(z)=\mathcal{L}_{\beta}(z \wedge n)$, so that we still have $\mathcal{L}_{\beta_{n}} \leq(r-1) \beta_{n}$, and $\beta_{n}^{\prime}(z) / z$ is bounded.

Now in both cases it is sufficient to apply Gronwall's lemma to the differential inequality (4.22) with $\beta=\beta_{n}$ and then let $n \rightarrow \infty$ to conclude with Fatou's lemma.

The correspondent inequalities for $\beta(z)=\left(z^{-}\right)^{r}$ are settled similarly.

Finally, the assumption (4.21) can be removed considering the solutions $u_{t}^{\varepsilon}$ relative to the same initial condition and to the derivations

$$
\boldsymbol{b}_{t}^{\varepsilon}:= \begin{cases}\boldsymbol{b}_{t} & \text { if } t \in[\epsilon, T) \\ 0 & \text { if } t \in(0, \epsilon) .\end{cases}
$$

Since $u_{t}^{\varepsilon}$ coincides with $\mathrm{P}_{\sigma t} \bar{u}$ for $t \in(0, \epsilon)$, (4.21) is fulfilled. Then, we can take weak limits in $L_{t}^{\infty}\left(L_{x}^{p} \cap L_{x}^{q}\right) \cap L^{2}(I ; \mathbb{V})$ as $\varepsilon \downarrow 0$ to obtain a function $u$ satisfying the desired properties.

\subsection{Vanishing viscosity and proof of Theorem 4.3}

Let $\boldsymbol{b}=\left(\boldsymbol{b}_{t}\right)_{t \in I}$ and $\bar{u} \in L^{1} \cap L^{r}(\mathfrak{m})(r \geq 2)$ satisfy the assumptions of Theorem 4.3. Let $\delta>0$, let $\rho$ be a mollifying kernel in $C_{c}^{1}(0,1)$ and set $\boldsymbol{b}_{t}^{\delta}:=\int_{0}^{1} \boldsymbol{b}_{t+s \delta} \rho(s) d s$ (where $\boldsymbol{b}_{t}=0$ for $t>T$ ) i.e. we let

$$
\varphi \mapsto d \varphi\left(\boldsymbol{b}_{t}^{\delta}\right)=\int_{0}^{1} d \varphi\left(\boldsymbol{b}_{t+s \delta}\right) \rho(s) d s .
$$

Since $|\boldsymbol{b}| \in L_{t}^{1}\left(L_{x}^{2}\right), \operatorname{div} \boldsymbol{b} \in L_{t}^{1}\left(L_{x}^{2}+L_{x}^{\infty}\right)$, it follows that $\left|\boldsymbol{b}^{\delta}\right| \in L_{t}^{\infty}\left(L_{x}^{2}\right), \operatorname{div} \boldsymbol{b}^{\delta} \in L_{t}^{\infty}\left(L_{x}^{2}+L_{x}^{\infty}\right)$ and the assumption $\operatorname{div} \boldsymbol{b}^{-} \in L_{t}^{1}\left(L_{x}^{1} \cap L_{x}^{\infty}\right)$ entails $\left(\operatorname{div} \boldsymbol{b}^{\delta}\right)^{-} \in L_{t}^{\infty}\left(L_{x}^{1} \cap L_{x}^{\infty}\right)$. Moreover, as $\delta \downarrow 0, d \varphi\left(\boldsymbol{b}^{\delta}\right)$ converges to $d \varphi(\boldsymbol{b})$ in $L_{t}^{1}\left(L_{x}^{2}+L_{x}^{\infty}\right)$, for every $\varphi \in \mathscr{A}$ and $\left\|\left(\operatorname{div} \boldsymbol{b}^{\delta}\right)^{-}\right\|_{1, \infty}$ converge to $\left\|(\operatorname{div} \boldsymbol{b})^{-}\right\|_{1, \infty}$. 
For fixed $\delta>0$, consider a sequence $u^{n}$ of solutions to (4.13) with $\boldsymbol{b}^{\delta}$ in place of $\boldsymbol{b}, \sigma=1 / n$, $n \geq 2$, as provided by Theorem 4.6 with $p=1$ and $q=r$, and notice that (4.7) gives

$$
\frac{1}{n}\left\|e^{-(1+\lambda) t} u^{n}\right\|_{L^{2}(I ; \mathbb{V})} \leq\|\bar{u}\|_{2}
$$

so that $v^{n}:=u^{n} / n$ is bounded in $L^{2}(I ; \mathbb{V})$. We would like to pass to the limit as $n \rightarrow \infty$ in

$$
\int_{0}^{T} \int-\left[\partial_{t} \varphi_{t}+d \varphi_{t}\left(\boldsymbol{b}_{t}\right)\right] u_{t}^{n}+\Gamma\left(\varphi_{t}, v_{t}^{n}\right) d \mathfrak{m} d t=\int \varphi_{0} \bar{u}^{\delta} d \mathfrak{m} \quad \forall \varphi \in \Phi_{\mathscr{A}}
$$

Inequality (4.15) entails that $\left(u^{n}\right)$ is bounded in $L_{t}^{\infty}\left(L_{x}^{1} \cap L_{x}^{r}\right)$ and so $v^{n}$ weakly converges to 0 in $L^{2}(I ; \mathbb{V})$. In addition, there exists a subsequence $n(k)$ such that $\left(u^{n(k)}\right)$ converges, in duality with $L_{t}^{1}\left(L_{x}^{2}+L_{x}^{\infty}\right)$, to some $u:=u^{\delta} \in L_{t}^{\infty}\left(L_{x}^{1} \cap L_{x}^{r}\right)$. This gives that $u^{\delta}$ is a weak solution to the continuity equation with $\boldsymbol{b}^{\delta}$ in place of $\boldsymbol{b}$.

We then let $\delta \downarrow 0$ and extract again a subsequence $\delta(k)$ such that $\left(u^{\delta(k)}\right)$ converge, in duality with $L_{t}^{1}\left(L_{x}^{r^{\prime}}+L_{x}^{\infty}\right)$, to some $u:=L_{t}^{\infty}\left(L_{x}^{1} \cap L_{x}^{r}\right)$ and is a weak solution to the continuity equation, thus concluding the proof of Theorem 4.3, except for conservation of mass.

Finally, we prove conservation of mass for any weak solution to the continuity equation, assuming existence of $f_{n} \in \mathscr{A}$ as in (4.3). The proof is based on the simple observation that our assumptions on $\boldsymbol{b}$ and $u$ imply $\boldsymbol{c}:=u \boldsymbol{b} \in L_{t}^{1}\left(L_{x}^{1}\right)$, and therefore

$$
\lim _{n \rightarrow \infty} \int_{0}^{T} \int\left|d f_{n}\left(\boldsymbol{c}_{t}\right)\right| d \mathfrak{m} d t=0
$$

Since

$$
\lim _{n \rightarrow \infty} \int u_{t} f_{n} d \mathfrak{m}=\int u_{t} d \mathfrak{m} \quad \forall t \in[0, T) \quad \text { and } \quad \frac{d}{d t} \int u_{t} f_{n} d \mathfrak{m}=\int d f_{n}\left(\boldsymbol{c}_{t}\right) d \mathfrak{m},
$$

we conclude that $\int u_{t} d \mathfrak{m}=\int \bar{u} d \mathfrak{m}$ for all $t \in[0, T)$.

\section{Uniqueness of solutions to the continuity equation}

In this section, we provide conditions that ensure uniqueness, in certain classes, for the continuity equation: these involve further regularity of $\boldsymbol{b}$, expressed in terms of bounds on its divergence and its deformation (introduced below), density assumptions of $\mathscr{A}$ in $\mathbb{V}_{p}$ and the validity of inequalities which correspond, in the smooth setting, to integral bounds on the gradient of the kernel of $P$.

Definition 5.1 ( $L^{p}-\Gamma$ inequality). Let $p \in[1, \infty]$. We say that the $L^{p}-\Gamma$ inequality holds if there exists $c_{p}>0$ satisfying

$$
\left\|\sqrt{\Gamma\left(\mathrm{P}_{t} f\right)}\right\|_{p} \leq \frac{c_{p}}{\sqrt{t}}\|f\|_{p}, \quad \text { for every } f \in L^{2} \cap L^{p}(\mathfrak{m}), t \in(0,1) .
$$

Although the $L^{p}-\Gamma$ inequality is expressed for $t \in(0,1)$, from its validity and $L^{p}$ contractivity of $\mathrm{P}$, we easily deduce that

$$
\left\|\sqrt{\Gamma\left(\mathrm{P}_{t} f\right)}\right\|_{p} \leq c_{p}(t \wedge 1)^{-1 / 2}\|f\|_{p}, \quad \text { for every } f \in L^{2} \cap L^{p}(\mathfrak{m}), t \in(0, \infty) .
$$


Notice also that, thanks to (2.12), the $L^{2}-\Gamma$ inequality always holds, with $c_{2}=1 / \sqrt{2}$. By semilinear Marcinkiewicz interpolation, we obtain that if the $L^{p}-\Gamma$ inequality holds then, for every $q$ between 2 and $p$, the $L^{q}-\Gamma$ inequality holds as well.

Definition 5.2 (Derivations with deformations of type $(r, s)$ ). Let $q \in[1, \infty]$, let $\boldsymbol{b}$ be $a$ derivation in $L^{q}+L^{\infty}$, with $\operatorname{div} \boldsymbol{b} \in L^{q}(\mathfrak{m})+L^{\infty}(\mathfrak{m})$, let $r, s \in[1, \infty]$ with $q^{-1}+r^{-1}+s^{-1}=1$ and assume that $\mathscr{A}$ is dense both in $\mathbb{V}_{r}$ and in $\mathbb{V}_{s}$. We say that the deformation of $\boldsymbol{b}$ is of type $(r, s)$ if there exists $c \geq 0$ satisfying

$$
\left|\int D \boldsymbol{b}^{\text {sym }}(f, g) d \mathfrak{m}\right| \leq c\|\sqrt{\Gamma(f)}\|_{r}\|\sqrt{\Gamma(g)}\|_{s}
$$

for all $f \in \mathbb{V}_{r}$ with $\Delta f \in L^{r} \cap L^{2}(\mathfrak{m})$ and all $g \in \mathbb{V}_{s}$ with $\Delta g \in L^{s} \cap L^{2}(\mathfrak{m})$, where

$$
\int D \boldsymbol{b}^{s y m}(f, g) d \mathfrak{m}:=-\frac{1}{2} \int d f(\boldsymbol{b}) \Delta g+d g(\boldsymbol{b}) \Delta f-(\operatorname{div} \boldsymbol{b}) \Gamma(f, g) d \mathfrak{m} .
$$

We let $\left\|D \boldsymbol{b}^{\text {sym }}\right\|_{r, s}$ be the smallest constant $c$ in (5.2).

The density assumption of $\mathscr{A}$ in $\mathbb{V}_{r}$ and $\mathbb{V}_{s}$ is necessary to extend the derivation $\boldsymbol{b}$ to all of $\mathbb{V}_{r}$ and $\mathbb{V}_{s}$, by Remark 3.7. Notice that the expression $\int D \boldsymbol{b}^{\text {sym }}(f, g) d \mathfrak{m}$ is symmetric with respect to $f, g$, so it is the role of $r$ and $s$ above can be interchanged.

Remark 5.3 (Deformation in the smooth case). Let $(X,\langle\cdot, \cdot\rangle)$ be a compact Riemannian manifold, let $\mathfrak{m}$ be its associated Riemannian volume and let $\Gamma(f, g)=\langle\nabla f, \nabla g\rangle$. Let $d f(\boldsymbol{b})=$ $\langle b, \nabla f\rangle$ for some smooth vector field $b$ and let $D b$ be the covariant derivative of $b$. The expression

$$
\langle\nabla g, \nabla\langle b, \nabla f\rangle\rangle+\langle\nabla f, \nabla\langle b, \nabla g\rangle\rangle-\langle b, \nabla\langle\nabla f, \nabla g\rangle\rangle=\langle D b \nabla g, \nabla f\rangle+\langle D b \nabla f, \nabla g\rangle
$$

gives exactly twice the symmetric part of the tensor $D b$, i.e. $2\left\langle D b^{\text {sym }} f, g\right\rangle$. Integrating over $X$ and then integrating by parts, we obtain twice the expression in (5.3), so that the derivation $\boldsymbol{b}$ associated to a smooth field $b$ is of type $(r, s)$ if $\left|D b^{\text {sym }}\right| \in L^{q}(\mathfrak{m})$, where $q \in[1, \infty]$ satisfies $q^{-1}+r^{-1}+s^{-1}=1$.

Theorem 5.4 (Uniqueness of solutions). Let $1<s \leq r<\infty, q \in(1, \infty]$ satisfy $q^{-1}+r^{-1}+$ $s^{-1}=1$. Assume the existence of $\left(f_{n}\right) \subset \mathscr{A}$ as in (4.3) and that, for $p \in\{r, s\}, \mathscr{A}$ is dense in $\mathbb{V}_{p}$ and the $L^{p}-\Gamma$ inequality holds. Let $\boldsymbol{b}=\left(\boldsymbol{b}_{t}\right)_{t \in(0, T)}$ be a Borel family of derivations, with

$$
|\boldsymbol{b}| \in L_{t}^{1}\left(L_{x}^{q}+L_{x}^{\infty}\right), \operatorname{div} \boldsymbol{b} \in L_{t}^{1}\left(L_{x}^{q}+L_{x}^{\infty}\right) \text { and }\left\|D^{s y m} \boldsymbol{b}_{t}\right\|_{r, s} \in L^{1}(0, T) .
$$

Then, there exists at most one weak solution $u$ in $(0, T) \times X$ to the continuity equation $\frac{d}{d t} u_{t}+\operatorname{div}\left(u_{t} \boldsymbol{b}_{t}\right)=0$ in the class

$$
\left\{u \in L_{t}^{\infty}\left(L_{x}^{r} \cap L_{x}^{2}\right): t \mapsto u_{t} \text { is weakly continuous in }[0, T)\right\},
$$

for every initial condition $\bar{u} \in L^{r} \cap L^{2}(\mathfrak{m})$.

The proof of this result is given in Section 5.2 and relies upon the strong convergence to 0 as $\alpha \downarrow 0$ of the commutator between divergence and action of the semigroup

$$
\mathscr{C}^{\alpha}\left(u_{t}, \boldsymbol{b}_{t}\right):=\operatorname{div}\left(\left(\mathrm{P}_{\alpha} u_{t}\right) \boldsymbol{b}_{t}\right)-\mathrm{P}_{\alpha}\left(\operatorname{div}\left(u_{t} \boldsymbol{b}_{t}\right)\right),
$$

proved in Lemma 5.8 in the next Section. We end this section with some comments on the density assumption on $\mathscr{A}$. 
Remark 5.5 (On the density of $\mathscr{A}$ in $\mathbb{V}_{p}$ ). The assumption that $\mathscr{A} \subset \mathbb{V}_{p}$ is dense for $p \in\{r, s\}$ is fundamental to show that the semigroup approximation $t \mapsto \mathrm{P}_{\alpha} u_{t}$ is a solution to another continuity equation, (5.14) below. This follows by the extension of the derivation on $\mathbb{V}_{p}$ provided by Remark 3.7. One could argue that the weak "Feller" condition (2.20) is sufficient to define $\boldsymbol{b}\left(\mathrm{P}_{\alpha} f\right)$, whenever $f \in \mathscr{A}$ : indeed Theorem 5.4 holds, assuming (2.20) in place of the density of $\mathscr{A}$ in $\mathbb{V}_{p}$, and the same proof goes through, with minor modifications (e.g. in Definition 5.2 above we require $f, g \in \mathscr{A}$ ). In view of Remark 2.4, one could also wonder whether $(2.20)$ and the $L^{p}$ - $\Gamma$ inequality are sufficient to entail density in $\mathbb{V}_{p}$ : the next lemma provides a partial affirmative answer.

Lemma 5.6. Let $p \in[2, \infty)$, assume that $(2.20)$ and the $L^{p}-\Gamma$ inequality hold and that

$$
\underset{t \downarrow 0}{\limsup }\left\|\sqrt{\Gamma\left(\mathrm{P}_{t} f\right)}\right\|_{p} \leq\|\sqrt{\Gamma(f)}\|_{p}, \quad \text { for every } f \in \mathbb{V}_{p} .
$$

Then, $\mathscr{A}$ is dense in $\mathbb{V}_{p}$.

Proof. Let $f \in \mathbb{V}_{p}$. Notice first that, since $\mathrm{P}_{t} f$ converge to $f$ in $\mathbb{V}$ as $t \downarrow 0$, Fatou's lemma gives

$$
\|\sqrt{\Gamma(f)}\|_{p} \leq \liminf _{t \downarrow 0}\left\|\sqrt{\Gamma\left(\mathrm{P}_{t} f\right)}\right\|_{p}
$$

which combined with (5.5) gives convergence of $\Gamma\left(\mathrm{P}_{t} f\right)^{1 / 2}$ to $\Gamma(f)^{1 / 2}$ in $L^{p}(\mathfrak{m})$.

To prove density, we let $f \in \mathbb{V}_{p}$ and consider the functions $\Phi_{n}: \mathbb{R} \rightarrow \mathbb{R}$, with derivative $\phi_{n}$, introduced in Lemma 2.2: since, by the chain rule, $\Phi_{n}(f)$ converge to $f$ in $\mathbb{V}_{p}$, it is sufficient to approximate each $\Phi_{n}(f)$ in $\mathbb{V}_{p}$ with elements of $\mathscr{A}$.

We first show that $\lim _{t \downarrow 0} \Phi_{n}\left(\mathrm{P}_{t} f\right)=\Phi_{n}(f)$ in $\mathbb{V}_{p}$. Since convergences in $\mathbb{V}$ and in $L^{p}(\mathfrak{m})$ are obvious, we prove $\Gamma\left(\Phi_{n}\left(\mathrm{P}_{t} f\right)-\Phi_{n}(f)\right)^{1 / 2} \rightarrow 0$ in $L^{p}(\mathfrak{m})$. We let $h_{1}=\mathrm{P}_{t} f$ and $h_{2}=f$ in (2.19) to get

$$
\begin{aligned}
\Gamma\left(\Phi_{n}\left(\mathrm{P}_{t} f\right)-\Phi_{n}(f)\right)^{1 / 2} & \leq\left|\phi_{n}\left(\mathrm{P}_{t} f\right)-\phi_{n}(f)\right| \Gamma(f)^{1 / 4} \Gamma\left(\mathrm{P}_{t} f\right)^{1 / 4} \\
& +2\left|\phi_{n}\left(\mathrm{P}_{t} f\right)-\phi_{n}(f)\right|^{1 / 2} \Gamma(f)^{1 / 4}\left(\Gamma(f)^{1 / 4}+\Gamma\left(\mathrm{P}_{t} f\right)^{1 / 4}\right) \\
& +\phi_{n}(f) \Gamma\left(\mathrm{P}_{t} f-f\right)^{1 / 2} .
\end{aligned}
$$

To handle the integral of the $p$-power of the last term in the right hand side, we notice that, since $\Gamma\left(\mathrm{P}_{t} f\right)^{1 / 2}$ converge to $\Gamma(f)^{1 / 2}$ in $L^{p}(\mathfrak{m})$, they converge also in $L^{p}\left(\mathfrak{m}^{\prime}\right)$ with $\mathfrak{m}^{\prime}=$ $\phi_{n}(f)^{p} \mathfrak{m}$. Since $\mathfrak{m}^{\prime}$ is finite we obtain that $\Gamma\left(\mathrm{P}_{t} f\right)^{p / 2}$ are equi-integrable with respect to $\mathfrak{m}^{\prime}$ and the Lebesgue-Vitali convergence ensures convergence to 0 . The first term can be handled similarly, adding and subtracting $\left|\phi_{n}\left(\mathrm{P}_{t} f\right)-\phi_{n}(f)\right|^{p} \Gamma(f)^{p / 4} \Gamma(f)^{p / 4}$ and using the dominated convergence, since $0 \leq \phi_{n} \leq 1$; the integral of the $p$-th power of the second term can be estimated with dominated convergence for $\int\left|\phi_{n}\left(\mathrm{P}_{t} f\right)-\phi_{n}(f)\right|^{p / 2} \Gamma(f)^{p / 2} d \mathfrak{m}$ and with the same argument we used for the first term for $\int\left|\phi_{n}\left(\mathrm{P}_{t} f\right)-\phi_{n}(f)\right|^{p / 2} \Gamma(f)^{p / 4} \Gamma\left(\mathrm{P}_{t} f\right)^{p / 4} d \mathfrak{m}$.

We proceed then to approximate $\Phi_{n}\left(\mathrm{P}_{t} f\right)$ in $\mathbb{V}_{p}$ by elements of $\mathscr{A}$, at fixed $n \geq 1$ and $t>0$. Let $\left(f_{k}\right) \subset \mathscr{A}$ be converging to $f$ in $L^{2} \cap L^{p}(\mathfrak{m})$. We show that $\Phi_{n}\left(\mathrm{P}_{t} f_{k}\right)$ converge to $\Phi_{n}\left(\mathrm{P}_{t} f\right)$ in $\mathbb{V}_{p}$. Notice that $\Phi_{n}\left(\mathrm{P}_{t} f_{k}\right)$ belong to $\mathscr{A}$, because of (2.20) and (2.17). Since convergence in $L^{2} \cap L^{p}(\mathfrak{m})$ holds, convergence in $\mathbb{V}_{p}$ follows again by (2.19) with $h_{1}=\mathrm{P}_{t} f_{k}$ and $h_{2}=\mathrm{P}_{t} f$, because

$$
\begin{aligned}
\Gamma\left(\Phi_{n}\left(\mathrm{P}_{t} f_{k}\right)-\Phi_{n}\left(\mathrm{P}_{t} f\right)\right)^{1 / 2} \leq & \left|\phi_{n}\left(\mathrm{P}_{t} f_{k}\right)-\phi_{n}\left(\mathrm{P}_{t} f\right)\right| \Gamma\left(\mathrm{P}_{t} f\right)^{1 / 4} \Gamma\left(\mathrm{P}_{t} f_{k}\right)^{1 / 4} \\
& +2\left|\phi_{n}\left(\mathrm{P}_{t} f_{k}\right)-\phi_{n}\left(\mathrm{P}_{t} f\right)\right|^{1 / 2} \Gamma\left(\mathrm{P}_{t} f\right)^{1 / 4}\left(\Gamma\left(\mathrm{P}_{t} f_{k}\right)^{1 / 4}+\Gamma\left(\mathrm{P}_{t} f\right)^{1 / 4}\right) \\
& +\phi_{n}\left(\mathrm{P}_{t} f\right) \Gamma\left(\mathrm{P}_{t} f_{k}-\mathrm{P}_{t} f\right)^{1 / 2} .
\end{aligned}
$$


By the $L^{2}-\Gamma$ inequality and the $L^{p}-\Gamma$ inequality, $\Gamma\left(\mathrm{P}_{t} f_{k}\right)^{1 / 2}$ converges to $\Gamma\left(\mathrm{P}_{t} f\right)^{1 / 2}$ in $L^{2} \cap$ $L^{p}(\mathfrak{m})$ as $k \rightarrow \infty$ and we can argue as we did in connection with (5.6) to obtain that $\Gamma\left(\Phi_{n}\left(\mathrm{P}_{t} f_{k}\right)-\Phi_{n}\left(\mathrm{P}_{t} f\right)\right)^{1 / 2} \rightarrow 0$ in $L^{p}(\mathfrak{m})$.

Actually, the proof above entails the following result. Let $p \in[1, \infty)$, assume that the $L^{p}-\Gamma$ inequality holds, and let $\mathscr{A} \subset \mathbb{V}$ satisfy (2.17), (2.20), (5.5), and dense in $L^{2} \cap L^{p}(\mathfrak{m})$. Then $\mathscr{A}$ is dense in $\mathbb{V}_{p}$. Finally, notice that this gives another proof of Remark 2.4.

\subsection{The commutator lemma}

We first collect some easy consequences of the $L^{r}$ - $\Gamma$ inequality, which allows for an approximation of the derivation $\boldsymbol{b}$, via the action of $\mathrm{P}_{\alpha}$, as expressed in the next proposition. We denote by $\boldsymbol{B}^{\alpha}$ the linear operator thus obtained, to stress the fact that it is not a derivation.

Proposition 5.7. Let $r, s \in(1, \infty), q \in(1, \infty]$ satisfy $q^{-1}+r^{-1}+s^{-1}=1$. Let $\boldsymbol{b}$ be a derivation in $L^{q}+L^{\infty}$ assume that $\mathscr{A} \subset \mathbb{V}_{r}$ is dense and that the $L^{r}-\Gamma$ inequality holds.

(i) For every $\alpha \in(0, \infty)$, the map

$$
\mathscr{A} \ni f \mapsto d\left(\mathrm{P}_{\alpha} f\right)(\boldsymbol{b})
$$

extends uniquely to $\boldsymbol{B}^{\alpha} \in \mathscr{L}\left(L^{r} \cap L^{2}(\mathfrak{m}), L^{s^{\prime}}(\mathfrak{m})+L^{2}(\mathfrak{m})\right)$, with

$$
\left\|\boldsymbol{B}^{\alpha}\right\| \leq \max \left\{c_{r}, c_{2}\right\}(\alpha \wedge 1)^{-1 / 2}\|\boldsymbol{b}\|_{L^{q}+L^{\infty}} .
$$

(ii) For all $f \in L^{r} \cap L^{2}(\mathfrak{m})$ the map $\alpha \mapsto \boldsymbol{B}^{\alpha}(f)$ is continuous from $(0, \infty)$ to $L^{s^{\prime}}(\mathfrak{m})+L^{2}(\mathfrak{m})$ and, if $\Delta f \in L^{r} \cap L^{2}(\mathfrak{m})$, it is $C^{1}\left((0, \infty) ; L^{s^{\prime}}(\mathfrak{m})+L^{2}(\mathfrak{m})\right)$, with

$$
\frac{d}{d \alpha} \boldsymbol{B}^{\alpha}(f)=\boldsymbol{B}^{\alpha}(\Delta f)
$$

(iii) Assume that $u \in L^{r} \cap L^{2}(\mathfrak{m}), \operatorname{div} \boldsymbol{b} \in L^{q}(\mathfrak{m})+L^{\infty}(\mathfrak{m})$. Then,

$$
\operatorname{div}\left(\beta\left(\mathrm{P}_{\alpha} u\right) \boldsymbol{b}\right)=\beta\left(\mathrm{P}_{\alpha} u\right) \operatorname{div} \boldsymbol{b}+\beta^{\prime}\left(\mathrm{P}_{\alpha} u\right) \boldsymbol{B}^{\alpha}(u) \in L^{s^{\prime}}(\mathfrak{m})+L^{2}(\mathfrak{m})
$$

for all $\alpha>0$ and all $\beta \in C^{1}(\mathbb{R}) \cap \operatorname{Lip}(\mathbb{R})$ with $\beta(0)=0$. In particular (5.8) with $\beta(z)=z$ gives

$$
\operatorname{div}\left(\left(\mathrm{P}_{\alpha} u\right) \boldsymbol{b}\right)=\left(\mathrm{P}_{\alpha} u\right) \operatorname{div} \boldsymbol{b}+\boldsymbol{B}^{\alpha}(u) \in L^{s^{\prime}}(\mathfrak{m})+L^{2}(\mathfrak{m}) .
$$

(iv) Assume $u \in L^{r} \cap L^{2}(\mathfrak{m})$ and $\operatorname{div} \boldsymbol{b} \in L^{q}(\mathfrak{m})+L^{\infty}(\mathfrak{m})$. Then $\mathscr{C}^{\alpha}\left(\mathrm{P}_{\delta} u, \boldsymbol{b}\right) \in L^{s^{\prime}}(\mathfrak{m})+L^{2}(\mathfrak{m})$ for every $\delta>0$ and

$$
\lim _{\alpha \downarrow 0}\left\|\mathscr{C}^{\alpha}\left(\mathrm{P}_{\delta} u, \boldsymbol{b}\right)\right\|_{L^{s^{\prime}}+L^{2}}=0
$$

Proof. (i). By Remark 3.7, if $\boldsymbol{c}$ is a derivation in $L^{q}$, then we can extend it to a linear operator on $\mathbb{V}_{r}$, thus $d\left(\mathrm{P}_{\alpha}\right)(\boldsymbol{c})$ is well defined. Since the $L^{r}$ - $\Gamma$ inequality holds, for every $f \in \mathscr{A}$, we get

$$
\left\|d\left(\mathrm{P}_{\alpha} f\right)(\boldsymbol{c})\right\|_{s^{\prime}} \leq\|\boldsymbol{c}\|_{q}\left\|\sqrt{\Gamma\left(\mathrm{P}_{\alpha} f\right)}\right\|_{r} \leq c_{r}(\alpha \wedge 1)^{-1 / 2}\|\boldsymbol{c}\|_{q}\|f\|_{r} .
$$


Analogously, if $\boldsymbol{c}$ is a derivation in $L^{\infty}, d\left(\mathrm{P}_{\alpha} f\right)(\boldsymbol{c})$ is well defined and there holds

$$
\left\|d\left(\mathrm{P}_{\alpha} f\right)(\boldsymbol{c})\right\|_{2} \leq\|\boldsymbol{c}\|_{\infty}\left\|\sqrt{\Gamma\left(\mathrm{P}_{\alpha} f\right)}\right\|_{2} \leq c_{2}(\alpha \wedge 1)^{-1 / 2}\|\boldsymbol{c}\|_{\infty}\|f\|_{2} .
$$

This gives $\left\|\boldsymbol{B}^{\alpha}(f)\right\|_{L^{s^{\prime}}+L^{2}} \leq \max \left\{c_{r}, c_{2}\right\}(\alpha \wedge 1)^{-1 / 2}\|\boldsymbol{b}\|_{L^{q}+L^{\infty}}\|f\|_{L^{r} \cap L^{2}}$ on $\mathscr{A}$. By density of $\mathscr{A}$ in $L^{r} \cap L^{2}(\mathfrak{m})$, this provides the existence of $\boldsymbol{B}^{s}$ and the estimate on its norm.

(ii). The semigroup law and the uniqueness of the extension give

$$
\boldsymbol{B}^{\alpha+\sigma}(f)=\boldsymbol{B}^{\alpha}\left(\mathrm{P}_{\sigma} f\right), \text { for every } f \in L^{r} \cap L^{2}(\mathfrak{m}), \alpha, \sigma \in(0, \infty) .
$$

Then, continuity follows easily, combining identity with (5.7) and the strong continuity of $\mathrm{P}_{s}$ :

$$
\left\|\boldsymbol{B}^{\alpha+\sigma}(f)-\boldsymbol{B}^{\alpha}(f)\right\|_{L^{s^{\prime}}+L^{2}} \leq \max \left\{c_{r}, c_{2}\right\}(\alpha \wedge 1)^{-1 / 2}\|\boldsymbol{b}\|_{L^{q}+L^{\infty}}\left\|\mathrm{P}_{\sigma} f-f\right\|_{L^{r} \cap L^{2}} .
$$

A similar argument shows differentiability if $\Delta f \in L^{r} \cap L^{2}(\mathfrak{m})$.

(iii). We obtain (5.9) by (3.3). By the chain rule, the identity (5.8) follows.

(iv). To prove that $\mathscr{C}^{\alpha}\left(\mathrm{P}_{\delta} u, \boldsymbol{b}\right) \in L^{s^{\prime}}(\mathfrak{m})+L^{2}(\mathfrak{m})$, it is sufficient to apply (5.9) twice, to get

$$
-\mathscr{C}^{\alpha}\left(\mathrm{P}_{\delta} u, \boldsymbol{b}\right)=\mathrm{P}_{\alpha}\left[\left(\mathrm{P}_{\delta} u\right) \operatorname{div} \boldsymbol{b}\right]+\mathrm{P}_{\alpha}\left(\boldsymbol{B}^{\delta}(u)\right)-\left(\mathrm{P}_{\alpha+\delta} u\right) \operatorname{div} \boldsymbol{b}-\boldsymbol{B}^{\delta+\delta}(u) \in L^{s^{\prime}}(\mathfrak{m})+L^{2}(\mathfrak{m}) .
$$

By strong continuity of $\alpha \mapsto \mathrm{P}_{\alpha}$ at $\alpha=0$ and continuity of $\alpha \mapsto \boldsymbol{B}^{\alpha}(u)$ in $(0, \infty)$, the same expression shows that $\mathscr{C}^{\alpha}\left(\mathrm{P}_{\delta} u, \boldsymbol{b}\right) \rightarrow 0$ in $L^{s^{\prime}}(\mathfrak{m})+L^{2}(\mathfrak{m})$ as $\alpha \downarrow 0$.

We are now in a position to state and prove the following crucial lemma.

Lemma 5.8 (Commutator estimate). Let $r, s \in(1, \infty), q \in(1, \infty]$ satisfy $q^{-1}+r^{-1}+s^{-1}=1$. Let $\boldsymbol{b}$ be a derivation in $L^{q}+L^{\infty}$ of type $(r, s)$ with $\operatorname{div} \boldsymbol{b} \in L^{q}(\mathfrak{m})+L^{\infty}(\mathfrak{m})$. Assume that $\mathscr{A}$ is dense in $\mathbb{V}_{p}$ and that the $L^{p}-\Gamma$ inequality holds, for $p \in\{r, s\}$. Then

$$
\left\|\mathscr{C}^{\alpha}(u, \boldsymbol{b})\right\|_{L^{s^{\prime}}+L^{2}} \leq c\|u\|_{L^{r} \cap L^{2}}\left[\left\|D^{s y m} \boldsymbol{b}\right\|_{r, s}+\|\operatorname{div} \boldsymbol{b}\|_{L^{q}+L^{\infty}}\right]
$$

for all $u \in L^{r} \cap L^{2}(\mathfrak{m})$, and all $\alpha \in(0,1)$, where $c$ is a constant depending only on the constants $c_{r}, c_{s}$ in (5.1) and the constants $c_{r}^{\Delta}$ and $c_{s}^{\Delta}$ in (2.14).

Moreover, $\mathscr{C}^{\alpha}(u, \boldsymbol{b}) \rightarrow 0$ in $L^{s^{\prime}}(\mathfrak{m})+L^{2}(\mathfrak{m})$ as $\alpha \downarrow 0$.

Proof. For brevity, we introduce the notation $g^{\alpha}:=\mathrm{P}_{\alpha} g$. By duality and density, inequality (5.11) is equivalent to the validity of

$$
\int d f^{\alpha}(\boldsymbol{b}) u d \mathfrak{m}-\int d f(\boldsymbol{b}) u^{\alpha} d \mathfrak{m} \leq c\left[\left\|D^{s y m} \boldsymbol{b}\right\|_{r, s}+\|\operatorname{div} b\|_{L^{q}+L^{\infty}}\right]\|u\|_{L^{r} \cap L^{2}}\|f\|_{L^{s} \cap L^{2}},
$$

for every $f$ of the form $f=\mathrm{P}_{\varepsilon} \varphi$, for some $\varphi \in \mathscr{A}, \varepsilon>0$. Since both sides are continuous in $u$ with respect to $L^{r} \cap L^{2}(\mathfrak{m})$ convergence, it is also enough to establish it in a dense set: we let therefore $u=\mathrm{P}_{\delta} v$ for some $v \in \mathscr{A}, \delta>0$.

We also notice that, by Proposition 5.7, we know that for such a choice of $u, \mathscr{C}^{\alpha}(u, \boldsymbol{b}) \rightarrow 0$ in $L^{s^{\prime}}(\mathfrak{m})+L^{2}(\mathfrak{m})$ as $\alpha \downarrow 0$. Thus, once (5.11) is obtained, the same convergence as $\alpha \downarrow 0$ holds for every $u \in L^{r} \cap L^{2}(\mathfrak{m})$, from a standard density argument. 
Then, we have to estimate

$$
\int d f^{\alpha}(\boldsymbol{b}) u d \mathfrak{m}-\int d f(\boldsymbol{b}) u^{\alpha} d \mathfrak{m}=F(\alpha)-F(0),
$$

where we let $F(\sigma)=\int d f^{\sigma}(\boldsymbol{b}) u^{\alpha-\sigma} d \mathfrak{m}$, for $\sigma \in[0, \alpha]$. Our assumption on $f=\mathrm{P}_{\varepsilon} \varphi$ entails, via Proposition 5.7, that the map $\sigma \mapsto d f^{\sigma}(\boldsymbol{b})=\boldsymbol{B}^{\varepsilon}\left(\varphi^{\sigma}\right)$ is $C^{1}\left([0, \alpha], L^{s^{\prime}}(\mathfrak{m})+L^{2}(\mathfrak{m})\right)$, with

$$
\frac{d}{d \sigma}\left[d f^{\sigma}(\boldsymbol{b})\right]=\boldsymbol{B}^{\varepsilon}\left(\Delta \varphi^{\sigma}\right)
$$

On the other hand, (2.14) entails that $\Delta u=\Delta \mathrm{P}_{\delta} v \in L^{r} \cap L^{2}(\mathfrak{m})$ and so $\sigma \mapsto u^{\sigma}$ in $C^{1}\left([0, \alpha], L^{r} \cap L^{2}(\mathfrak{m})\right)$. Thus, we are in a position to apply Leibniz rule to obtain

$$
F(\alpha)-F(0)=\int_{0}^{\alpha}\left(\int \boldsymbol{B}^{\varepsilon}\left(\Delta \varphi^{\sigma}\right) u^{\alpha-\sigma}-d f^{\sigma}(\boldsymbol{b}) \Delta u^{\alpha-\sigma} d \mathfrak{m}\right) d \sigma .
$$

By applying (5.9) with $\Delta \varphi^{\sigma}$ in place of $u$, we integrate by parts to obtain

$$
\int \boldsymbol{B}^{\varepsilon}\left(\Delta \varphi^{\sigma}\right) u^{\alpha-\sigma} d \mathfrak{m}=-\int \Delta f^{\sigma} d u^{\alpha-\sigma}(\boldsymbol{b})+(\operatorname{div} \boldsymbol{b})\left(\Delta f^{\sigma}\right) u^{\alpha-\sigma} d \mathfrak{m} .
$$

We now estimate separately the terms

$$
I=-\int \Delta f^{\sigma} d u^{\alpha-\sigma}(\boldsymbol{b})+d f^{\sigma}(\boldsymbol{b}) \Delta u^{\alpha-\sigma} d \mathfrak{m}, \quad I I:=-\int(\operatorname{div} \boldsymbol{b})\left(\Delta f^{\sigma}\right) u^{\alpha-\sigma} d \mathfrak{m},
$$

at fixed $\sigma \in(0, \alpha)$ and then integrate over $\sigma$.

To handle the first term, we add and subtract $\int(\operatorname{div} \boldsymbol{b}) \Gamma\left(f^{\sigma}, u^{\alpha-\sigma}\right) d \mathfrak{m}$, and thus recognize twice the deformation of $\boldsymbol{b}$, applied to $f^{\sigma}$ and $u^{\alpha-\sigma}$, which are admissible functions in the sense of Definition 5.2, because of (5.1) and (2.14):

$$
I=2 \int D^{s y m} \boldsymbol{b}\left(f^{\sigma}, u^{\alpha-\sigma}\right) d \mathfrak{m}-\int(\operatorname{div} \boldsymbol{b}) \Gamma\left(f^{\sigma}, u^{\alpha-\sigma}\right) d \mathfrak{m} .
$$

We use the assumption on $D^{s y m} \boldsymbol{b}, \operatorname{div} \boldsymbol{b}$ and $L^{r}-\Gamma$ and $L^{s}-\Gamma$ as well as $L^{2}-\Gamma$ inequalities to obtain that

$$
|I| \leq\left[2\left\|D^{s y m} \boldsymbol{b}\right\|_{r, s}+\|\operatorname{div} \boldsymbol{b}\|_{L^{q}+L^{\infty}}\right] \frac{c}{\sqrt{\alpha(\alpha-\sigma)}}\|f\|_{L^{s} \cap L^{2}}\|u\|_{L^{r} \cap L^{2}}
$$

with $c=c_{r}+c_{s}+c_{2}$. To handle integration over $\sigma \in(0, \alpha)$, we use

$$
\int_{0}^{\alpha} \frac{d \sigma}{\sqrt{\sigma(\alpha-\sigma)}}=\pi .
$$

To estimate the second term, we add and subtract

$$
\int(\operatorname{div} \boldsymbol{b})\left(\Delta f^{\sigma}\right) u^{\alpha} d \mathfrak{m}=\frac{d}{d \sigma} \int(\operatorname{div} \boldsymbol{b}) f^{\sigma} u^{\alpha} d \mathfrak{m},
$$

obtaining

$$
I I=\int(\operatorname{div} \boldsymbol{b})\left(\Delta f^{\sigma}\right)\left(u^{\alpha}-u^{\alpha-\sigma}\right) d \mathfrak{m}-\frac{d}{d \sigma} \int(\operatorname{div} \boldsymbol{b}) f^{\sigma} u^{s} d \mathfrak{m}
$$


We then estimate the first part of II by means of (2.14) and Corollary 2.1, to get

$$
\frac{c^{\Delta}}{\sigma} \min \left\{2, c^{\Delta} \log \left(1+\frac{\sigma}{\alpha-\sigma}\right)\right\}\|f\|_{L^{s} \cap L^{2}}\|u\|_{L^{r} \cap L^{2}}
$$

with $c^{\Delta}=c_{s}^{\Delta}+c_{r}^{\Delta}+c_{2}^{\Delta}$.

The remaining part of $I I$ is estimated once we integrate over $\sigma \in(0, \alpha)$, as

$$
-\int_{0}^{\alpha} \frac{d}{d \sigma} \int(\operatorname{div} \boldsymbol{b}) f^{\sigma} u^{\alpha} d \mathfrak{m} d \sigma=\int \operatorname{div} \boldsymbol{b}\left(f-f^{\alpha}\right) u^{\alpha} \leq 2\|\operatorname{div} \boldsymbol{b}\|_{L^{q}+L^{\infty}}\|f\|_{L^{s} \cap L^{2}}\|u\|_{L^{r} \cap L^{2}} .
$$

To conclude, we notice that

$$
\begin{aligned}
\int_{0}^{\alpha} \min \left\{\frac{2}{\sigma}, \frac{c^{\Delta}}{\sigma} \log \left(1+\frac{\sigma}{\alpha-\sigma}\right)\right\} d \sigma & \leq \max \left\{2, c^{\Delta}\right\} \int_{0}^{\alpha} \min \left\{\frac{1}{\sigma}, \frac{1}{\alpha-\sigma}\right\} d \sigma \\
& =2 \log 2 \max \left\{2, c^{\Delta}\right\}
\end{aligned}
$$

thus the proof of $(5.12)$ is complete.

Remark 5.9 (Time-dependent commutator estimate). By integrating the commutator estimate with respect to time, we can achieve a similar estimate for time-dependent derivations $\boldsymbol{b}$ of type $(r, s)$ satisfying

$$
|\boldsymbol{b}| \in L_{t}^{1}\left(L_{x}^{q}+L_{x}^{\infty}\right), \operatorname{div} \boldsymbol{b} \in L_{t}^{1}\left(L_{x}^{q}+L_{x}^{\infty}\right) \text { and }\left\|D^{s y m} \boldsymbol{b}_{t}\right\|_{r, s} \in L^{1}(I),
$$

still assuming the validity of the $L^{p}-\Gamma$ inequalities for $p \in\{r, s\}$ :

$$
\int_{I}\left\|\mathscr{C}^{\alpha}\left(u_{t}, \boldsymbol{b}_{t}\right)\right\|_{L^{s^{\prime}}+L^{2}} d t \leq c\|u\|_{L_{t}^{\infty}\left(L_{x}^{r} \cap L_{x}^{2}\right)}\left[\int_{I}\left\|D^{s y m} \boldsymbol{b}_{t}\right\|_{r, s}+\left\|\operatorname{div} \boldsymbol{b}_{t}\right\|_{L^{q}+L^{\infty}} d t\right]
$$

for all $u \in L_{t}^{\infty}\left(L_{x}^{r} \cap L_{x}^{2}\right)$ and $\alpha \in(0, \infty)$. Moreover, dominated convergence gives

$$
\lim _{\alpha \downarrow 0} \int_{I}\left\|\mathscr{C}^{\alpha}\left(u_{t}, \boldsymbol{b}_{t}\right)\right\|_{L^{s^{\prime}}+L^{2}} d t=0 .
$$

\subsection{Proof of Theorem 5.4}

The proof of Theorem 5.4 is similar to that of Theorem 4.6, but it crucially exploits Lemma 5.8 to show that the error terms are negligible.

Let $\left(f_{n}\right) \subset \mathscr{A}$ be a sequence given by (4.3). Starting from $|z|^{1+r / s}$, we define $\beta$ as in (4.19), namely

$$
\beta(z):= \begin{cases}1+\frac{r+s}{s}(z-1) & \text { if } z>1 \\ |z|^{1+r / s} & \text { if }|z| \leq 1 \\ 1-\frac{r+s}{s}(z+1) & \text { if } z<-1\end{cases}
$$

so that $\mathcal{L}_{\beta} \leq(r / s) \beta$ and $\beta$ has linear growth at infinity.

By the linearity of the equation we can assume $\bar{u}=0$ and the goal is to prove that $u=0$. We first extend the time interval $I=(0, T)$ to $(-1, T)$, setting $\boldsymbol{b}_{t}=0$ for $t \in(-1,0)$ and 
given the weakly continuous (in duality with $\mathscr{A}$ ) solution in $[0, T)$, with $u \in L^{\infty}\left(L_{x}^{r} \cap L_{x}^{2}\right)$, we extend it to a weakly continuous solution in $(-1, T)$, setting $u_{t}=0$ for $t \in(-1,0)$.

For every $\alpha>0$, let $u_{t}^{\alpha}=\mathrm{P}_{\alpha} u_{t} \in L^{\infty}\left(L_{x}^{r} \cap L_{x}^{2}\right)$. As in the proof of Theorem 4.6, replacing $\varphi$ in (4.16) by $\mathrm{P}_{s} \varphi$ (recall Remark 5.5) we can check that $t \mapsto u_{t}^{\alpha}$ is a weakly continuous solution to the continuity equation

$$
\partial_{t} u_{t}^{\alpha}+\operatorname{div}\left(u_{t}^{\alpha} \boldsymbol{b}_{t}\right)=\mathscr{C}^{\alpha}\left(u_{t}, \boldsymbol{b}_{t}\right)
$$

By (5.9) in Proposition 5.7 and (5.11) in Lemma 5.8, this equation entails that

$$
\frac{d}{d t} u_{t}^{\alpha}=\mathscr{C}^{\alpha}\left(u_{t}, \boldsymbol{b}_{t}\right)-\operatorname{div}\left(u_{t}^{\alpha} \boldsymbol{b}_{t}\right) \in L_{t}^{1}\left(L_{x}^{s^{\prime}}+L_{x}^{2}\right)
$$

for a.e. $t \in(-1, T)$. Since $t \mapsto \int f_{n} \beta\left(u_{t}^{\alpha}\right) d \mathfrak{m}$ is lower semicontinuous (because $\beta$ is convex and $t \mapsto u_{t}$ is weakly continuous) and since $\left|\beta^{\prime}(z)\right| \sim|z|^{r / s}$ near the origin and $r \geq s$ imply that $\beta^{\prime}\left(u_{t}^{\alpha}\right)$ is uniformly bounded in $L^{s} \cap L^{2}(\mathfrak{m})$, we can argue as in the proof of (4.23) to obtain that $t \mapsto \int f_{n} \beta\left(u_{t}^{\alpha}\right) d \mathfrak{m}$ is absolutely continuous and

$$
\frac{d}{d t} \int f_{n} \beta\left(u_{t}^{\alpha}\right) d \mathfrak{m}=\int f_{n} \beta^{\prime}\left(u_{t}^{\alpha}\right) \frac{d}{d t} u_{t}^{\alpha} d \mathfrak{m}=\int f_{n} \beta^{\prime}\left(u_{t}^{\alpha}\right) \mathscr{C}^{\alpha}\left(u_{t}^{\alpha}, \boldsymbol{b}_{t}\right)-f_{n} \beta^{\prime}\left(u_{t}^{\alpha}\right) \operatorname{div}\left(u_{t}^{\alpha} \boldsymbol{b}_{t}\right) d \mathfrak{m}
$$

for a.e. $t \in I$. Now, setting $\Psi_{n}(t, \alpha):=\int f_{n} \beta\left(u_{t}^{\alpha}\right) d \mathfrak{m}$, identities (5.8) and (5.9) in Proposition 5.7 give

$$
\frac{d}{d t} \Psi_{n}(t, \alpha)=\int f_{n} \beta^{\prime}\left(u_{t}^{\alpha}\right) \mathscr{C}^{\alpha}\left(u_{t}^{\alpha}, \boldsymbol{b}_{t}\right) d \mathfrak{m}-\int f_{n} \operatorname{div}\left(\beta\left(u_{t}^{\alpha}\right) \boldsymbol{b}_{t}\right)+f_{n} \mathcal{L}_{\beta}\left(u_{t}^{\alpha}\right) \operatorname{div} \boldsymbol{b}_{t} d \mathfrak{m}
$$

for a.e. $t \in I$. Hence, denoting $L_{t}:=(r / s)\left\|\operatorname{div} \boldsymbol{b}_{t}^{-}\right\|_{\infty} \in L^{1}(-1, T)$, we can use the inequality $\mathcal{L}_{\beta} \leq(r / s) \beta$ to get

$$
\frac{d}{d t} \Psi_{n}(t, \alpha) \leq L_{t} \Psi_{n}(t, \alpha)+\int f_{n} \beta^{\prime}\left(u_{t}^{\alpha}\right) \mathscr{C}^{\alpha}\left(u_{t}^{\alpha}, \boldsymbol{b}_{t}\right) d \mathfrak{m}+\int \beta\left(u_{t}^{\alpha}\right) d f_{n}\left(\boldsymbol{b}_{t}\right) d \mathfrak{m} .
$$

Now we let $\alpha \downarrow 0$ and use the strong convergence of commutators in $L^{s^{\prime}}(\mathfrak{m})+L^{2}(\mathfrak{m})$ and the boundedness of $\beta^{\prime}\left(u_{t}^{\alpha}\right)$ in $L^{s} \cap L^{2}(\mathfrak{m})$ to obtain that $t \mapsto \int_{X} f_{n} \beta\left(u_{t}\right)$ is absolutely continuous, and that

$$
\frac{d}{d t} \int_{X} f_{n} \beta\left(u_{t}\right) d \mathfrak{m} \leq L_{t} \int_{X} f_{n} \beta\left(u_{t}\right) d \mathfrak{m}+\int \beta\left(u_{t}\right) d f_{n}\left(\boldsymbol{b}_{t}\right) d \mathfrak{m} .
$$

By integration, taking into account that $\int_{X} f_{n} \beta\left(u_{t}\right) d \mathfrak{m} \equiv 0$ on $(-1,0)$, we get

$\log \left(\frac{1}{\delta} \int_{X} f_{n} \beta\left(u_{t}\right) d \mathfrak{m}+1\right) \leq\|L\|_{1}+\int_{0}^{T} \int \beta\left(u_{s}\right) d f_{n}\left(\boldsymbol{b}_{s}\right) d \mathfrak{m} d s \quad$ for all $t \in(-1, T)$ and all $\delta>0$.

Eventually we use (4.3) and the monotone convergence theorem to obtain

$$
\log \left(\frac{1}{\delta} \int_{X} \beta\left(u_{t}\right) d \mathfrak{m}+1\right) \leq\|L\|_{1} \quad \text { for all } t \in(-1, T) \text { and } \delta>0 .
$$

Letting $\delta \downarrow 0$ gives $u=0$. 


\section{Curvature assumptions and their implications}

In this section we add to the basic setting (2.1) a suitable curvature condition, and see the implication of this assumption on the structural conditions of density of $\mathscr{A}$ in the spaces $\mathbb{V}_{p}$ and the existence of $f_{n} \in \mathscr{A}$ in (4.3) made in the previous sections.

In the sequel $K$ denotes a generic but fixed real number, and $\mathrm{I}_{K}$ denotes the real function

$$
\mathrm{I}_{K}(t):=\int_{0}^{t} \mathrm{e}^{K r} d r= \begin{cases}\frac{1}{K}\left(\mathrm{e}^{K t}-1\right) & \text { if } K \neq 0 \\ t & \text { if } K=0\end{cases}
$$

Definition 6.1 (Bakry-Émery conditions). We say that $\mathrm{BE}_{2}(K, \infty)$ holds if

$$
\Gamma\left(\mathrm{P}_{t} f\right) \leq \mathrm{e}^{-2 K t} \mathrm{P}_{t}(\Gamma(f)) \quad \mathfrak{m} \text {-a.e. in } X \text {, for every } f \in \mathbb{V}, t \geq 0 \text {. }
$$

We say that $\mathrm{BE}_{1}(K, \infty)$ holds if

$$
\sqrt{\Gamma\left(\mathrm{P}_{t} f\right)} \leq \mathrm{e}^{-K t} \mathrm{P}_{t}(\sqrt{\Gamma(f)}) \quad \mathfrak{m} \text {-a.e. in } X \text {, for every } f \in \mathbb{V}, t \geq 0 \text {. }
$$

We stated both the curvature conditions for the sake of completeness only, but we remark that $\mathrm{BE}_{2}(K, \infty)$ is sufficient for many of the results we are interested in this section. Obviously, $\mathrm{BE}_{1}(K, \infty)$ implies $\mathrm{BE}_{2}(K, \infty)$; the converse, first proved by Bakry in [Bak85], has been recently extended to a nonsmooth setting by Savaré (see [Sav13, Corollary 3.5]) under the assumption that $\mathcal{E}$ is quasi-regular. The quasi-regularity property has many equivalent characterizations, a transparent one is for instance in terms of the existence of a sequence of compact sets $F_{k} \subset X$ such that

$$
\bigcup_{k}\left\{f \in \mathbb{V}: f=0 \text { m-a.e. in } X \backslash F_{k}\right\}
$$

is dense in $\mathbb{V}$.

The validity of the following inequality is actually equivalent to $\mathrm{BE}_{2}(K, \infty)$, see for instance [AGS12, Corollary 2.3] for a proof.

Proposition 6.2 (Reverse Poincaré inequalities). If $\mathrm{BE}_{2}(K, \infty)$ holds, then

$$
2 \mathrm{I}_{2 K}(t) \Gamma\left(\mathrm{P}_{t} f\right) \leq \mathrm{P}_{t} f^{2}-\left(\mathrm{P}_{t} f\right)^{2} \quad \mathfrak{m} \text {-a.e. in } X,
$$

for all $t>0, f \in L^{2}(\mathfrak{m})$.

Corollary 6.3 ( $L^{p}-\Gamma$ inequalities). If $\mathrm{BE}_{2}(K, \infty)$ holds, then $L^{p}-\Gamma$ inequalities hold for $p \in$ $[2, \infty]$.

Proof. The validity of $L^{p}-\Gamma$ inequalities for $p \in[2, \infty]$ is obtained integrating (6.3),

$$
\left(2 \mathrm{I}_{2 K}(t)\right)^{p / 2} \int \Gamma\left(\mathrm{P}_{t} f\right)^{p / 2} d \mathfrak{m} \leq \int\left(\mathrm{P}_{t} f^{2}\right)^{p / 2} d \mathfrak{m} \leq \int f^{p} d \mathfrak{m}
$$

and using $2 \mathrm{I}_{2 K}(t)^{-1}=O\left(t^{-1}\right)$ as $t \downarrow 0$.

Another consequence of $\mathrm{BE}_{2}(K, \infty)$ is the following higher integrability of $\Gamma(f)$, recently proved in [AMS13, Thm. 3.1] assuming higher integrability of $f$ and $\Delta f$. 
Theorem 6.4 (Gradient interpolation). Assume that $\mathrm{BE}_{2}(K, \infty)$ holds and let $\lambda \geq K^{-}$, $f \in L^{2} \cap L^{\infty}(\mathfrak{m})$. If $p \in\{2, \infty\}$ and $\Delta f \in L^{p}(\mathfrak{m})$, then $\Gamma(f) \in L^{p}(\mathfrak{m})$ and

$$
\|\Gamma(f)\|_{p} \leq c\|f\|_{\infty}\|\Delta f+\lambda f\|_{p}
$$

for a universal constant $c$ (i.e. independent of $\lambda, K, X, \mathfrak{m})$.

Finally, we will need two more consequences of the $\mathrm{BE}_{2}(K, \infty)$ condition, proved under the quasi-regularity assumption in [Sav13]: the first one, first proved in [Sav13, Lemma 3.2] and then slightly improved in [AMS13, Thm. 5.5], is the implication

$$
f \in \mathbb{V}, \quad \Delta f \in L^{4}(\mathfrak{m}) \quad \Longrightarrow \quad \Gamma(f) \in \mathbb{V} .
$$

In particular, this implication provides $L^{4}$ integrability of $\sqrt{\Gamma(f)}$, consistently with the integrability of the Laplacian. Moreover, it will be particularly useful the quantitative estimate, first proved in [Sav13, Thm. 3.4] and then slightly improved in [AMS13, Corollary 5.7]:

$$
\Gamma(\Gamma(f)) \leq 4 \gamma_{2, K}[f] \Gamma(f) \quad \mathfrak{m} \text {-a.e. in } X \text {, whenever } f \in \mathbb{V}, \Delta f \in L^{4}(\mathfrak{m}) .
$$

The function $\gamma_{2, K}[f]$ in (6.6) is nonnegative, it satisfies the $L^{1}$ estimate

$$
\int \gamma_{2, K}[f] d \mathfrak{m} \leq \int_{X}\left((\Delta f)^{2}-K \Gamma(f)\right) d \mathfrak{m}
$$

and it can be represented as the density w.r.t. $\mathfrak{m}$ of the nonnegative (and possibly singular w.r.t. $\mathfrak{m})$ measure defined by

$$
\mathbb{V} \ni \varphi \mapsto \int_{X}-\frac{1}{2} \Gamma(\Gamma(f), \varphi)+\Delta f \Gamma(f, \varphi)+\left((\Delta f)^{2}-K \Gamma(f)\right) \varphi d \mathfrak{m}
$$

The nonnegativity of this measure is one of the equivalent formulations of $\mathrm{BE}_{2}(K, \infty)$, see $[$ Sav13, §3] for a more detailed discussion.

\subsection{Choice of the algebra $\mathscr{A}$}

We first prove that the following "minimal" choice for the algebra $\mathscr{A}$ provides $(2.16),(2.17)$ and optimal density conditions.

Proposition 6.5. Under assumption $\mathrm{BE}_{2}(K, \infty)$, the algebra

$$
\mathscr{A}_{1}:=\left\{f \in \bigcap_{1 \leq p \leq \infty} L^{p}(\mathfrak{m}): f \in \mathbb{V}, \sqrt{\Gamma(f)} \in \bigcap_{1 \leq p \leq \infty} L^{p}(\mathfrak{m})\right\}
$$

satisfies (2.16), (2.17) and it is dense in every space $\mathbb{V}_{p}$, for $p \in[1, \infty)$.

Proof. Since (2.17) is obviously satisfied by the chain rule, we need only to show density of $\mathscr{A}_{1}$. First, we consider the algebra $\mathcal{A}=\mathbb{V}_{2} \cap \mathbb{V}_{\infty}$, which satisfies the weak "Feller" condition (2.20) because of (6.1). Moreover, for $p \in[2, \infty)$, the validity of the $L^{p}$ - $\Gamma$ inequality entail that $\mathcal{A}$ is dense in $L^{2} \cap L^{p}$, and taking the $L^{p / 2}$ norm in (6.1) gives that (5.5) holds. By Lemma 5.6 (actually, the remark below its proof) we conclude that $\mathcal{A}$ is dense in $\mathbb{V}_{p}$, for every $p \in[2, \infty)$.

To establish density of $\mathscr{A}_{1}$ in $\mathbb{V}_{p}$ for $p \in[1, \infty)$ it is sufficient to notice that the "refining" procedure in Lemma 2.2 applied to $\mathcal{A}$ preserves all the densities in $\mathbb{V}_{p}$ for $p \in[2, \infty)$, and provides an algebra contained in $\mathscr{A}_{1}$.

Retaining the density condition and the algebra property, one can also consider classes smaller than $\mathscr{A}_{1}$, including for instance bounds in $L^{p}(\mathfrak{m})$ for the Laplacian. 


\subsection{Conservation of mass}

In this section we see prove that the curvature condition, together with the conservativity condition $\mathrm{P}_{t}^{\infty} 1=1$ for all $t>0$ (recall that $\mathrm{P}_{t}^{\infty}: L^{\infty}(\mathfrak{m}) \rightarrow L^{\infty}(\mathfrak{m})$ is the dual semigroup in (2.15)), imply the existence of a sequence $\left(f_{n}\right) \subset \mathscr{A}_{1}$ as in (4.3). Notice that the conservativity is loosely related to a mass conservation property, for the continuity equation with derivation induced by the logarithmic derivative of the density; therefore, even though sufficient conditions adapted to the prescribed derivation $\boldsymbol{b}$ could be considered as well, it is natural to consider the conservativity of $\mathrm{P}$ in connection with (4.3).

Proposition 6.6. If $\mathrm{BE}_{2}(K, \infty)$ holds and $\mathrm{P}$ is conservative, then there exist $\left(f_{n}\right) \subset \mathscr{A}_{1}$ satisfying (4.3).

Proof. Let $\left(g_{n}\right) \subset L^{1} \cap L^{\infty}(\mathfrak{m})$ be a non-decreasing sequence of functions (whose existence is ensured by the $\sigma$-finiteness assumption on $\mathfrak{m})$ with

$$
0 \leq g_{n} \leq 1 \text { for every } n \geq 1 \text { and } \lim _{n \rightarrow \infty} g_{n}=1 \text {, m-a.e. in } X \text {. }
$$

These conditions imply in particular that $g_{n} \rightarrow 1$ weakly* in $L^{\infty}(\mathfrak{m})$.

Let $h_{n}=\int_{0}^{1} \mathrm{P}_{s} g_{n} d s=\int_{0}^{1} \mathrm{P}_{s}^{\infty} g_{n} d s$ and define $f_{n}:=\mathrm{P}_{1} h_{n}=\mathrm{P}_{1}^{\infty} h_{n}$. By linearity and continuity of $\mathrm{P}^{\infty}$ we obtain that $f_{n} \rightarrow \mathrm{P}_{1}^{\infty} 1=1$ weakly* in $L^{\infty}(\mathfrak{m})$. In addition, expanding the squares, it is easily seen that

$$
\lim _{n \rightarrow \infty} \int\left(1-f_{n}\right)^{2} v d \mathfrak{m}=0 \quad \forall v \in L^{1}(\mathfrak{m}) .
$$

Hence, by a diagonal argument we can assume (possibly extracting a subsequence) that $f_{n} \rightarrow 1$ $\mathfrak{m}$-a.e. in $X$.

Since $h_{n} \leq 1$, the reverse Poincaré inequality (6.3) entails

$$
\Gamma\left(f_{n}\right) \leq \frac{\mathrm{P}_{1} h_{n}^{2}-\left(f_{n}\right)^{2}}{2 \mathrm{I}_{2 K}(1)} \leq \frac{1-\left(f_{n}\right)^{2}}{2 \mathrm{I}_{2 K}(1)}, \quad \text { m-a.e. in } X .
$$

Taking the square roots of both sides and using the a.e. convergence of $f_{n}$ we obtain, thanks to dominated convergence, that $\sqrt{\Gamma\left(f_{n}\right)}$ weakly* converge to 0 in $L^{\infty}(\mathfrak{m})$.

Finally, we discuss the regularity of $f_{n}$. Since

$$
\Delta f_{n}=\int_{1}^{2} \Delta \mathrm{P}_{s} g_{n} d s=\mathrm{P}_{2} g_{n}-\mathrm{P}_{1} g_{n} \in L^{\infty}(\mathfrak{m})
$$

we can use Theorem 6.4 to obtain $\sqrt{\Gamma\left(f_{n}\right)} \in L^{\infty}(\mathfrak{m})$. In order to obtain integrability of the gradient for powers between 1 and 2 we can replace $f_{n}$ by $k_{n}:=\Phi_{1}\left(f_{n}\right) / \Phi_{1}(1)$, with $\Phi_{1}: \mathbb{R} \rightarrow \mathbb{R}$ as introduced in Lemma 2.2 .

\subsection{Derivations associated to gradients and their deformation}

In this section, we study more in detail the class of "gradient" derivations $\boldsymbol{b}_{V}$ in (3.2). More generally, we analyze the regularity of the derivation $f \mapsto \omega \Gamma(f, V)$ associated to sufficiently regular $V$ and $\omega$ in $\mathbb{V}$.

For $p \in(1, \infty]$, let us denote

$$
D_{L^{p}}(\Delta):=\left\{f \in \mathbb{V} \cap L^{p}(\mathfrak{m}): \Delta f \in L^{p}(\mathfrak{m})\right\} .
$$


Thanks to the implication (6.5), $D_{L^{4}}(\Delta) \subset \mathbb{V}_{4}$ and the Hessian

$$
(f, g) \mapsto H[V](f, g):=\frac{1}{2}[\Gamma(f, \Gamma(V, g))+\Gamma(g, \Gamma(V, f))-\Gamma(V, \Gamma(f, g))] \in L^{1}(\mathfrak{m}),
$$

is well defined on $D_{L^{4}}(\Delta) \times D_{L^{4}}(\Delta)$. Notice that the expression is symmetric in $(f, g)$, that $(V, f, g) \mapsto H[V](f, g)$ is multilinear, and that

$$
H[V]\left(f, g_{1} g_{2}\right)=H[V]\left(f, g_{1}\right) g_{2}+g_{1} H[V]\left(f, g_{2}\right) .
$$

By [Sav13, Thm. 3.4], we have the estimate

$$
|H[V](f, g)| \leq \sqrt{\gamma_{2, K}[V]} \sqrt{\Gamma(f)} \sqrt{\Gamma(g)}, \quad \mathfrak{m} \text {-a.e. in } X,
$$

for every $f, g \in D_{L^{4}}(\Delta)$.

Theorem 6.7. If $\mathrm{BE}_{2}(K, \infty)$ holds and $\mathcal{E}$ is quasi-regular, then for all $V \in D(\Delta), \omega \in$ $\mathbb{V} \cap L^{\infty}(\mathfrak{m})$ with $\sqrt{\Gamma(\omega)} \in L^{\infty}(\mathfrak{m})$ and $c \in \mathbb{R}$, the derivation $\boldsymbol{b}=(\omega+c) \boldsymbol{b}_{V}$ has deformation of type $(4,4)$ according to Definition 5.2 with $q=2$, and it satisfies

$$
\left\|D^{\text {sym }} \boldsymbol{b}\right\|_{4,4} \leq\|\omega+c\|_{\infty}\left\|(\Delta V)^{2}-K \Gamma(V)\right\|_{1}+\|\sqrt{\Gamma(\omega)}\|_{\infty}\|\sqrt{\Gamma(V)}\|_{2} .
$$

Proof. Assume first that $V \in D_{L^{4}}(\Delta)$. Let $f, g \in D_{L^{4}}(\Delta)$. After integrating by parts the Laplacians of $f$ and $g$, the very definition of $D^{\text {sym }} \boldsymbol{b}$ gives

$$
\int D^{s y m} \boldsymbol{b}(f, g) d \mathfrak{m}=\int(\omega+c) H[V](f, g)+\frac{1}{2}[\Gamma(\omega, f) \Gamma(V, g)+\Gamma(\omega, g) \Gamma(V, f)] d \mathfrak{m} .
$$

By Hölder inequality, we can use (6.12) to estimate $\left|\int D^{s y m} \boldsymbol{b}(f, g) d \mathfrak{m}\right|$ from above with

$$
\left[\|\omega\|_{\infty}\left\|\sqrt{\gamma_{2, K}[V]}\right\|_{2}+\|\sqrt{\Gamma(\omega)}\|_{\infty}\|\sqrt{\Gamma(V)}\|_{2}\right]\|\sqrt{\Gamma(f)}\|_{4}\|\sqrt{\Gamma(g)}\|_{4} .
$$

Thus, by definition of $\left\|D^{s y m} \boldsymbol{b}\right\|_{4,4},(6.13)$ follows, taking also (6.7) into account. To pass to the general case $V \in D(\Delta)$, it is sufficient to approximate $V$ with $V_{n} \in D_{L^{4}}(\Delta)$ in such a way that $V_{n} \rightarrow V$ in $\mathbb{V}$ and $\Delta V_{n} \rightarrow \Delta V$ in $L^{2}(\mathfrak{m})$ and notice that $\int D^{\text {sym }} \boldsymbol{b}_{n}(f, g) d \mathfrak{m}$ converge to $\int D^{\text {sym }} \boldsymbol{b}(f, g) d \mathfrak{m}$ directly from (5.3). The existence of such an approximating sequence is obtained arguing as in [AMS13, Lemma 4.2], i.e. given $f \in D(\Delta)$, we let $h=f-\Delta f \in L^{2}(\mathfrak{m})$,

$$
h_{n}:=\max \{\min \{h, n\},-n\} \in L^{2} \cap L^{\infty}(\mathfrak{m})
$$

and define $f_{n}$ as the unique (weak) solution to $f_{n}-\Delta f_{n}=h_{n}$. The maximum principle for $\Delta$ (or equivalently the fact that the resolvent operator $R_{1}=(I-\Delta)^{-1}$ is Markov) gives $f_{n} \in L^{2} \cap L^{\infty}(\mathfrak{m})$, thus $\Delta f_{n} \in L^{2} \cap L^{\infty}(\mathfrak{m})$ and by $L^{2}$-continuity of $R_{1}$, as $n \rightarrow \infty$, both $h_{n}$ and $f_{n}$ converge, respectively towards $h$ and $f$. By difference, also $\Delta f_{n}$ converge towards $\Delta f$ in $L^{2}(\mathfrak{m})$ and this gives also easily convergence of $f_{n}$ to $f$ in $\mathbb{V}$.

We end this section with a technical result that will be useful when dealing with probability measures on vector spaces, in particular in Section 9.5. 
Proposition 6.8. Assume that $\mathfrak{m}(X)=1$, that $\mathrm{BE}_{2}(K, \infty)$ holds and that $\mathcal{E}$ is quasi-regular. Let $\left(V_{i}\right)_{i \geq 1} \subset D_{L^{4}}(\Delta)$ generate an algebra dense in $\mathbb{V}$ and satisfy $\Gamma\left(V_{i}, V_{j}\right)=\delta_{i, j} \mathfrak{m}$-a.e. in $X$. Then,

(a) $\Gamma(f)=\sum_{i \geq 1} \Gamma\left(V_{i}, f\right)^{2} \mathfrak{m}$-a.e. in $X$, for every $f \in \mathbb{V}$;

(b) $H\left[V_{i}\right]=0$ for every $i \geq 1$.

Moreover, for every $q \in[1, \infty]$ and $\left.b=\left(b^{i}\right) \in L^{q}\left(X ; \ell^{2}\right)\right)$ the derivation

$$
f \mapsto d f(\boldsymbol{b})=\sum_{i} b^{i} \Gamma\left(V_{i}, f\right)
$$

satisfies $|\boldsymbol{b}|^{2} \leq \sum_{i}\left|b^{i}\right|^{2}$ and therefore belongs to $L^{q}$. In addition, if $r, s \in[4, \infty)$, satisfy $q^{-1}+r^{-1}+s^{-1}=1, \operatorname{div} \boldsymbol{b} \in L^{q}(\mathfrak{m})$ and $b_{i} \in \mathbb{V}$ for every $i \geq 1$, then

$$
\left\|D^{s y m} \boldsymbol{b}\right\|_{r, s} \leq \frac{1}{2}\left\|\left(\sum_{i, j}\left|\Gamma\left(V_{j}, b_{i}\right)+\Gamma\left(V_{i}, b_{j}\right)\right|^{2}\right)^{1 / 2}\right\|_{q} .
$$

Proof. When $f=\psi\left(V_{1}, \ldots, V_{n}\right)$ belongs to the algebra generated by $\left(V_{i}\right)$, the first identity is immediate from $\Gamma\left(V_{i}, V_{j}\right)=\delta_{i, j}$. The general case of $(a)$ follows by density.

From the definition (6.11) of Hessian it holds $H\left[V_{i}\right]\left(V_{j}, V_{k}\right)=0$ for every $i, j, k \geq 1$. For fixed $i, j \geq 1$, the derivation $g \mapsto H\left[V_{i}\right]\left(V_{j}, g\right)$ belongs to $L^{2}(\mathfrak{m})$ in virtue of (6.12), thus it can be extended by density of $\mathscr{A}$ to all of $\mathbb{V}$. By the chain rule, the extended derivation is identically zero on the algebra generated by $\left(V_{i}\right)$ thus by density it is the null derivation. In particular, for $g \in \mathscr{A}, H\left[V_{i}\right]\left(V_{j}, g\right)=0$, for every $j \geq 1$. Keeping fixed $g \in \mathscr{A}$, we argue similarly, and obtain that $H\left[V_{i}\right](f, g)=0 \mathfrak{m}$-a.e. in $X$ for every $f, g \in \mathscr{A}$, thus proving (b).

If only a finite number of $b^{i}$ 's is different from 0 , and they belong to $\mathbb{V}$, the claimed estimate (6.15) follows immediately by linearity, (6.14) and (b) above. The general case follows by "cylindrical" approximation, where the assumption $r, s \geq 4$ play a role. Indeed, given $f \in \mathbb{V}_{r} \cap D_{L^{r}}(\Delta)$ and $g \in \mathbb{V}_{s} \cap D_{L^{s}}(\Delta)$ it holds $f, g \in D_{L^{4}}(\Delta)$, thus $\Gamma(f, g) \in \mathbb{V}$ and we can integrate by parts the last term in (5.3), obtaining

$$
\int D^{s y m} \boldsymbol{b}(f, g) d \mathfrak{m}=-\frac{1}{2} \int d f(\boldsymbol{b}) \Delta g+d g(\boldsymbol{b}) \Delta f+d(\Gamma(f, g))(\boldsymbol{b}) d \mathfrak{m} .
$$

Let $N \geq 1$ and let $\boldsymbol{b}_{N}$ be the derivation associated to the sequence $\left(b^{1}, \ldots, b^{N}, 0,0, \ldots\right)$. Given $h \in \mathbb{V}$, it holds

$$
\left|d(h) \boldsymbol{b}_{N}-d(h) \boldsymbol{b}\right| \leq \Gamma(h)^{1 / 2}\left(\sum_{i>N}\left|b^{i}\right|^{2}\right)^{1 / 2}, \quad \mathfrak{m} \text {-a.e. in } X .
$$

By this estimate with $h=f, h=g$ and $h=\Gamma(f, g)$, Hölder inequality and dominated convergence we conclude that the sequence $\int D^{s y m} \boldsymbol{b}_{N}(f, g) d \mathfrak{m}$ converge towards $\int D^{s y m} \boldsymbol{b}(f, g) d \mathfrak{m}$ as $N \rightarrow \infty$, entailing (6.15).

Notice that the assumption $r, s \in[4, \infty)$ is used only to obtain $\Gamma(f, g) \in \mathbb{V}$ and so (6.16). The same argument indeed shows that, for $r, s \in[1, \infty)$ and $q \in(1, \infty]$ with $q^{-1}+$ $r^{-1}+s^{-1}=1$, if $\mathscr{A}$ is dense in the space $\mathbb{V}_{p} \cap D_{L^{p}}(\Delta)$, endowed with the norm $\|f\|=$ $\|f\|_{\mathbb{V}_{p}}+\|\Delta f\|_{L^{2} \cap L^{p}}$, for $p \in\{r, s\}$ and it satisfies $\Gamma(f, g) \in \mathscr{A}$ for $f, g \in \mathscr{A}$, then the last statement in Proposition 6.8 holds, regardless of the condition $r, s \in[4, \infty)$. 


\section{The superposition principle in $\mathbb{R}^{\infty}$ and in metric measure spaces}

In this section we denote $\mathbb{R}^{\infty}=\mathbb{R}^{\mathbb{N}}$ endowed with the product topology and we shall denote by $\pi^{n}:=\left(p_{1}, \ldots, p_{n}\right): \mathbb{R}^{\infty} \rightarrow \mathbb{R}^{n}$ the canonical projections from $\mathbb{R}^{\infty}$ to $\mathbb{R}^{n}$. On the space $\mathbb{R}^{\infty}$ we consider the complete and separable distance

$$
d_{\infty}(x, y):=\sum_{n=1}^{\infty} 2^{-n} \min \left\{1,\left|p_{n}(x)-p_{n}(y)\right|\right\} .
$$

Accordingly, we consider the space $C\left([0, T] ; \mathbb{R}^{\infty}\right)$ endowed with the distance

$$
\delta(\eta, \tilde{\eta}):=\sum_{n=1}^{\infty} 2^{-n} \max _{t \in[0, T]} \min \left\{1,\left|p_{n}(\eta(t))-p_{n}(\tilde{\eta}(t))\right|\right\},
$$

which makes $C\left([0, T] ; \mathbb{R}^{\infty}\right)$ complete and separable as well. We shall also consider the subspace $A C_{w}\left([0, T] ; \mathbb{R}^{\infty}\right)$ of $C\left([0, T] ; \mathbb{R}^{\infty}\right)$ consisting of all $\eta$ such that $p_{i} \circ \eta \in A C([0, T])$ for all $i \geq 1$. Notice that for this class of curves the derivative $\eta^{\prime} \in \mathbb{R}^{\infty}$ can still defined a.e. in $(0, T)$, arguing componentwise. We use the notation $A C_{w}$ to avoid the confusion with the space of absolutely continuous maps from $[0, T]$ to $\left(\mathbb{R}^{\infty}, d_{\infty}\right)$.

It is immediate to check that for any convex choice of superlinear and l.s.c. functions $\Psi_{n}:[0, \infty) \rightarrow[0, \infty]$ and for l.s.c. functions $\Phi_{n}:[0, \infty) \rightarrow[0, \infty]$ with $\Phi_{n}(v) \rightarrow \infty$ as $v \rightarrow \infty$ the functional $\mathcal{A}: C\left([0, T] ; \mathbb{R}^{\infty}\right) \rightarrow[0, \infty]$ defined by

$$
\mathcal{A}(\eta):= \begin{cases}\sum_{n=1}^{\infty}\left[\Phi_{n}\left(p_{n} \circ \eta(0)\right)+\int_{0}^{T} \Psi_{n}\left(\left|\left(p_{n} \circ \eta\right)^{\prime}\right|\right) d t\right] & \text { if } \eta \in A C_{w}\left([0, T] ; \mathbb{R}^{\infty}\right) \\ \infty & \text { if } \eta \in C\left([0, T] ; \mathbb{R}^{\infty}\right) \backslash A C_{w}\left([0, T] ; \mathbb{R}^{\infty}\right)\end{cases}
$$

is coercive in $C\left([0, T] ; \mathbb{R}^{\infty}\right)$, i.e. all sublevels $\{\mathcal{A} \leq M\}$ are compact in $C\left([0, T] ; \mathbb{R}^{\infty}\right)$.

We call smooth cylindrical function any $f: \mathbb{R}^{\infty} \rightarrow \mathbb{R}$ representable in the form

$$
f(x)=\psi\left(\pi_{n}(x)\right)=\psi\left(p_{1}(x), \ldots, p_{n}(x)\right) \quad x \in \mathbb{R}^{\infty},
$$

with $\psi: \mathbb{R}^{n} \rightarrow \mathbb{R}$ bounded and continuously differentiable, with bounded derivative. When we want to emphasize $n$, we say that $f$ is $n$-cylindrical. Given $\psi$ smooth cylindrical, we define $\nabla f: \mathbb{R}^{\infty} \rightarrow c_{0}$ (where $c_{0}$ is the space of sequences $\left(x_{n}\right)$ null for $n$ large enough) by

$$
\nabla f(x):=\left(\frac{\partial \psi}{\partial z_{1}}\left(\pi_{n}(x)\right), \ldots, \frac{\partial \psi}{\partial z_{n}}\left(\pi_{n}(x)\right), 0,0, \ldots\right) .
$$

We fix a Borel vector field $c:(0, T) \times \mathbb{R}^{\infty} \rightarrow \mathbb{R}^{\infty}$ and a weakly continuous (in duality with smooth cylindrical functions) family of Borel probability measures $\left\{\nu_{t}\right\}_{t \in(0, T)}$ in $\mathbb{R}^{\infty}$ satisfying

$$
\int_{0}^{T} \int\left|p_{i}\left(\boldsymbol{c}_{t}\right)\right| d \nu_{t} d t<\infty, \quad \forall i \geq 1
$$

and, in the sense of distributions,

$$
\frac{d}{d t} \int f d \nu_{t}=\int\left(c_{t}, \nabla f\right) d \nu_{t} \quad \text { in }(0, T), \text { for all } f \text { smooth cylindrical. }
$$


Theorem 7.1 (Superposition principle in $\mathbb{R}^{\infty}$ ). Under assumptions (7.2) and (7.3), there exists a Borel probability measure $\boldsymbol{\lambda}$ in $C\left([0, T] ; \mathbb{R}^{\infty}\right)$ satisfying $\left(e_{t}\right)_{\#} \boldsymbol{\lambda}=\nu_{t}$ for all $t \in(0, T)$, concentrated on $\gamma \in A C_{w}\left([0, T] ; \mathbb{R}^{\infty}\right)$ which are solutions to the $O D E \dot{\gamma}=c_{t}(\gamma)$ a.e. in $(0, T)$.

Proof. The statement is known in finite-dimensional spaces, see e.g. [AGS05, Thm. 8.2.1] for the case when $\iint\left|\boldsymbol{c}_{t}\right|^{r} d \nu_{t} d t<\infty$ for some $r>1$, and [AC08, Thm. 12] for the case $r=1$ ). For $i \geq 1$ we choose convex, superlinear, l.s.c. functions $\Psi_{i}:[0, \infty) \rightarrow[0, \infty]$ with

$$
\int_{0}^{T} \int \Psi_{i}\left(\left|p_{i}\left(\boldsymbol{c}_{t}\right)\right|\right) d \nu_{t} d t \leq 2^{-i}
$$

and coercive $\Phi_{i}:[0, \infty) \rightarrow[0, \infty)$ satisfying

$$
\int \Phi_{i}\left(p_{i}(x)\right) d \mu_{0}(x) \leq 2^{-i}
$$

and define $\mathcal{A}$ accordingly.

Defining $\nu_{t}^{n}:=\left(\pi^{n}\right)_{\#} \nu_{t}$ and $\boldsymbol{c}_{t, i}^{n}, 1 \leq i \leq n$, as the density of $\left(e_{t}\right)_{\#}\left(p_{i}\left(\boldsymbol{c}_{t}\right) \nu_{t}\right)$ w.r.t. to $\nu_{t}^{n}$, it is immediate to check with Jensen's inequality that

$$
\int_{0}^{T} \int \Psi_{i}\left(\left|\boldsymbol{c}_{t, i}^{n}\right|\right) d \nu_{t}^{n} d t \leq \int_{0}^{T} \int \Psi_{i}\left(\left|p_{i}\left(\boldsymbol{c}_{t}\right)\right|\right) d \nu_{t} d t, \quad i \geq 1
$$

and that $\nu_{t}^{n}$ solve the continuity equation in $\mathbb{R}^{n}$ relative to the vector field $\boldsymbol{c}^{n}=\left(\boldsymbol{c}_{i}^{n}, \ldots, \boldsymbol{c}_{n}^{n}\right)$. Therefore the finite-dimensional statement provides $\boldsymbol{\lambda}_{n}$, probability measures in $C\left([0, T] ; \mathbb{R}^{n}\right)$, concentrated on absolutely continuous a.e. solutions to the ODE $\dot{\gamma}=\boldsymbol{c}_{t}^{n}(\gamma)$ and satisfying $\left(e_{t}\right)_{\#} \boldsymbol{\lambda}_{n}=\nu_{t}^{n}$ for all $t \in[0, T]$.

In order to pass to the limit as $n \rightarrow \infty$ it is convenient to view $\boldsymbol{\lambda}_{n}$ as probability measures in $C\left([0, T] ; \mathbb{R}^{\infty}\right)$ concentrated on curves $\gamma$ such that $p_{i}(\gamma)$ is null for $i>n$ and $\nu^{n}$ as probability measures in $\mathbb{R}^{\infty}$ concentrated on $\left\{x \in \mathbb{R}^{\infty}: p_{i}(x)=0 \forall i>n\right\} \subset c_{0}$. Accordingly, if we set $\boldsymbol{c}_{t, i}^{n} \equiv 0$ for $i>n$, we retain the property that $\boldsymbol{\lambda}_{n}$ is concentrated on absolutely continuous solutions to the ODE $\dot{\gamma}=\boldsymbol{c}_{t}^{n}(\gamma)$ and satisfies $\left(e_{t}\right)_{\#} \boldsymbol{\lambda}_{n}=\nu_{t}^{n}$ for all $t \in[0, T]$.

Using (7.6) and our choice of $\Psi_{i}$ and $\Phi_{i}$ we immediately obtain

$$
\int \mathcal{A}(\gamma) d \boldsymbol{\lambda}_{n}(\gamma) \leq 2
$$

hence the sequence $\left(\boldsymbol{\lambda}_{n}\right)$ is tight in $\mathscr{P}\left(C\left([0, T] ; \mathbb{R}^{\infty}\right)\right)$.

We claim that any limit point $\boldsymbol{\lambda}$ fulfills the properties stated in the lemma. Just for notational simplicity, we assume in the sequel that the whole family $\left(\boldsymbol{\lambda}_{n}\right)$ weakly converges to $\boldsymbol{\lambda}$. The lower semicontinuity of $\mathcal{A}$ gives $\int \mathcal{A} d \boldsymbol{\lambda}<\infty$, hence $\boldsymbol{\lambda}$ is concentrated on $A C_{w}\left([0, T] ; \mathbb{R}^{\infty}\right)$. Furthermore, since

$$
\gamma \mapsto \pi_{k} \circ \gamma(t), \quad t \in[0, T]
$$

are continuous from $C\left([0, T] ; \mathbb{R}^{\infty}\right)$ to $\mathbb{R}^{k}$, passing to the limit as $n \rightarrow \infty$ in the identity $\left(\pi_{k}\right)_{\sharp}\left(e_{t}\right)_{\sharp} \boldsymbol{\lambda}_{n}=\left(\pi_{k}\right)_{\sharp} \nu_{t}^{n}$ it follows that $\left(\pi_{k}\right)_{\sharp}\left(e_{t}\right)_{\sharp} \boldsymbol{\lambda}=\left(\pi_{k}\right)_{\sharp} \nu_{t}$ for all $k$. We can now use the fact that cylindrical functions generate the Borel $\sigma$-algebra of $\mathbb{R}^{\infty}$ to obtain that $\left(e_{t}\right)_{\sharp} \boldsymbol{\lambda}=\nu_{t}$.

It remains to prove that $\boldsymbol{\lambda}$ is concentrated on solutions to the ODE $\dot{\gamma}=\boldsymbol{c}_{t}(\gamma)$. To this aim, suffices to show that

$$
\int\left|p_{i} \circ \gamma(t)-p_{i} \circ \gamma(0)-\int_{0}^{t} p_{i} \circ \boldsymbol{c}_{s}(\gamma(s)) d s\right| d \boldsymbol{\lambda}(\gamma)=0
$$


for any $t \in[0, T]$ and $i \geq 1$. The technical difficulty is that this test function, due to the lack of regularity of $\boldsymbol{c}$, is not continuous in $C\left([0, T] ; \mathbb{R}^{\infty}\right)$. To this aim, we prove first that

$$
\int\left|p_{i} \circ \gamma(t)-p_{i} \circ \gamma(0)-\int_{0}^{t} \boldsymbol{d}_{s}(\gamma(s)) d s\right| d \boldsymbol{\lambda}(\gamma) \leq \int_{(0, T) \times \mathbb{R}^{\infty}}\left|p_{i} \circ \boldsymbol{c}-\boldsymbol{d}\right| d \nu_{t} d t
$$

for any bounded Borel function $\boldsymbol{d}$ with $\boldsymbol{d}(t, \cdot) k$-cylindrical for all $t \in(0, T)$, with $k$ independent of $t$. It is clear that the space

$$
\left\{\boldsymbol{d} \in L^{1}\left(\nu_{t} d t\right): \boldsymbol{d}(t, \cdot) \text { cylindrical for all } t \in(0, T)\right\}
$$

is dense in $L^{1}\left(\nu_{t} d t\right)$; by a further approximation, also the space

$$
\bigcup_{k=1}^{\infty}\left\{\boldsymbol{d} \in L^{1}\left(\nu_{t} d t\right): \boldsymbol{d}(t, \cdot) k \text {-cylindrical for all } t \in(0, T)\right\}
$$

is dense. Hence, choosing a sequence $\left(\boldsymbol{d}^{m}\right)$ of functions admissible for (7.8) converging to $p_{i} \circ \boldsymbol{c}$ in $L^{1}\left(\nu_{t} d t\right)$ and noticing that

$$
\int_{(0, T) \times \mathbb{R}^{\infty}}\left|p_{i} \circ \boldsymbol{c}_{s}(\gamma(s))-\boldsymbol{d}_{s}^{m}(\gamma(s))\right| d s d \boldsymbol{\lambda}(\gamma)=\int_{(0, T) \times \mathbb{R}^{\infty}}\left|p_{i} \circ \boldsymbol{c}-\boldsymbol{d}^{m}\right| d \nu_{t} d t \rightarrow 0,
$$

we can take the limit in (7.8) with $\boldsymbol{d}=\boldsymbol{d}^{m}$ to obtain (7.7).

It remains to show (7.8). We first prove

$$
\limsup _{n \rightarrow \infty} \int_{(0, T) \times \mathbb{R}^{\infty}}\left|p_{i} \circ \boldsymbol{c}^{n}-\boldsymbol{d}\right| d \nu_{s}^{n} d s \leq \int_{(0, T) \times \mathbb{R}^{\infty}}\left|p_{i} \circ \boldsymbol{c}-\boldsymbol{d}\right| d \nu_{t} d t
$$

for all bounded Borel functions $\boldsymbol{d}$ with $\boldsymbol{d}(t, \cdot) k$-cylindrical for all $t \in(0, T)$, with $k$ independent of $t$. The proof is elementary, because for $n \geq k$ and $t \in(0, T)$ there holds

$$
\left(p_{i} \circ \boldsymbol{c}_{t}^{n}-\boldsymbol{d}_{t}\right) \nu_{t}^{n}=\left(e_{n}\right)_{\#}\left(\left(p_{i} \circ \boldsymbol{c}_{t}-\boldsymbol{d}_{t}\right) \nu_{t}\right) .
$$

Now we can prove (7.8), with a limiting argument based on the fact that (7.7) holds for $c^{n}, \lambda_{n}$ :

$$
\begin{aligned}
& \int\left|p_{i} \circ \gamma(t)-p_{i} \circ \gamma(0)-\int_{0}^{t} \boldsymbol{d}_{s}(\gamma(s)) d s\right| d \boldsymbol{\lambda}_{n}(\gamma) \\
= & \int\left|\int_{0}^{t}\left(p_{i} \circ \boldsymbol{c}_{s}^{n}(\gamma(s))-\boldsymbol{d}_{s}(\gamma(s))\right) d s\right| d \boldsymbol{\lambda}_{n}(\gamma) \\
\leq & \iint_{0}^{t}\left|p_{i} \circ \boldsymbol{c}_{s}^{n}-\boldsymbol{d}_{s}\right|(\gamma(s)) d s d \boldsymbol{\lambda}_{n}(\gamma) \leq \int_{(0, T) \times \mathbb{R}^{\infty}}\left|p_{i} \circ \boldsymbol{c}^{n}-\boldsymbol{d}\right| d \nu_{s}^{n} d s .
\end{aligned}
$$

Since $\boldsymbol{d}_{s}(\cdot)$ is cylindrical for all $s$ and uniformly bounded w.r.t. $s$, the map

$$
\gamma \mapsto\left|p_{i} \circ \gamma(t)-p_{i} \circ \gamma(0)-\int_{0}^{t} \boldsymbol{d}_{s}(\gamma(s)) d s\right|
$$

belongs to $C\left(C\left([0, T] ; \mathbb{R}^{\infty}\right)\right)$ and is nonnegative. Hence, taking the limit in the chain of inequalities above and using (7.9) we obtain (7.8). 
We next consider the case of a (possibly extended) metric measure spaces $(X, \tau, \mathfrak{m}, d)$. Starting from the basic setup of Section 2, we have indeed only a topology $\tau$ and the measure $\mathfrak{m}$. We assume the existence of a countable set $\mathscr{A}^{*} \subset\left\{f \in \mathscr{A}:\|\Gamma(f)\|_{\infty} \leq 1\right\}$ satisfying:

$\mathbb{R} \mathscr{A}^{*}$ is dense in $\mathbb{V}$ and any function in $\mathscr{A}^{*}$ has a $\tau$-continuous representative,

$$
\exists \lim _{n \rightarrow \infty} f\left(x_{n}\right) \text { in } \mathbb{R} \text { for all } f \in \mathscr{A}^{*} \Longrightarrow \exists \lim _{n \rightarrow \infty} x_{n} \text { in } X
$$

Since supp $\mathfrak{m}=X$, the $\tau$-continuous representative of a $\mathfrak{m}$-measurable function if exists is unique, and for this reason we do not use in (7.12) and in the sequel a distinguished notation for the continuous representative of functions in $\mathscr{A}^{*}$. Notice that (7.11) implies that the family $\mathscr{A}^{*}$ separates the points of $X$ and that (7.10) and (7.11) can be easily fulfilled in many cases when a distance $d$ is a priori given, considering the distance functions from a countable and dense set of points, see Section 9.6 for more details.

Remark 7.2 (Extended distance induced by $\mathscr{A}^{*}$ ). Following [BM95] (see also [Stu95, Sto10]) we build $d_{\mathscr{A}^{*}}: X \times X \rightarrow[0, \infty]$ as

$$
d_{\mathscr{A}}(x, y)=\sup \left\{|f(x)-f(y)|: f \in \mathscr{A}^{*}\right\}, \quad x, y \in X .
$$

A priori, $d_{\mathscr{A}^{*}}$ is an extended distance in the sense of [AGS11a], since it may take the value $\infty$; nevertheless, by definition, all functions in $\mathscr{A}^{*}$ are 1-Lipschitz w.r.t $d_{\mathscr{A}^{*}}$ and $d_{\mathscr{A}} *$ is the smallest extended distance with this property. In particular the derivative $\frac{d}{d t}(f \circ \eta)$ which occurs in the next definition makes sense a.e. in $(0, T)$ when $f \in \mathscr{A}^{*}$ and $\eta \in A C\left([0, T] ;\left(X, d_{\mathscr{A}^{*}}\right)\right)$, because $f \circ \eta$ belongs to $A C([0, T])$. However, we will not use the topology induced by $d_{\mathscr{A}^{*}}$, which could be much finer than the topology $\tau$ and, in the next definition, we will require only continuity of $\eta:[0, T] \rightarrow X$ (with the topology $\tau$ in the target space $X$ ) and $W^{1,1}(0, T)$ regularity of $f \circ \eta$, for $f \in \mathscr{A}$. A posteriori, in Lemma 7.4 we are going to recover some absolute continuity for $\eta$, with respect to $d_{\mathscr{A}^{*}}$. In any case, whenever $f \in \mathscr{A}$ has a continuous representative (as it happens when $f \in \mathscr{A}^{*}$ ), the continuity of $f \circ \eta$ in conjunction with Sobolev regularity gives $f \circ \eta \in A C([0, T])$.

Definition 7.3 (ODE induced by a family $\left(\boldsymbol{b}_{t}\right)$ of derivations). Let $\boldsymbol{\eta} \in \mathscr{P}(C([0, T] ; X))$ and let $\left(\boldsymbol{b}_{t}\right)_{t \in(0, T)}$ be a Borel family of derivations. We say that $\boldsymbol{\eta}$ is concentrated on solutions to the $O D E \dot{\eta}=\boldsymbol{b}_{t}(\eta)$ if

$$
f \circ \eta \in W^{1,1}(0, T) \text { and } \frac{d}{d t}(f \circ \eta)(t)=d f\left(\boldsymbol{b}_{t}\right)(\eta(t)) \text { for a.e. } t \in(0, T) \text {, for } \boldsymbol{\eta} \text {-a.e. } \eta
$$

for all $f \in \mathscr{A}$.

Notice that the property of being concentrated on solutions to the ODE implicitly depends on the choice of Borel representatives of the maps $f$ and $(t, x) \mapsto d f\left(\boldsymbol{b}_{t}\right)(x), f \in \mathscr{A}$. As such, it should be handled with care. We will see, however, that in the class of regular flows of Definition 8.1 this sensitivity to the choice of Borel representatives disappears, see Remark 8.2.

The following simple lemma shows that time marginals of measures $\boldsymbol{\eta}$ concentrated on solutions to the ODE $\dot{\eta}=\boldsymbol{b}_{t}(\eta)$ provide weakly continuous solutions to the continuity equation.

Lemma 7.4. Let $\boldsymbol{\eta} \in \mathscr{P}(C([0, T] ; X))$ be concentrated on solutions $\eta$ to the ODE $\dot{\eta}=\boldsymbol{b}_{t}(\eta)$, where $|\boldsymbol{b}| \in L_{t}^{1}\left(L_{x}^{p}\right)$ for some $p \in[1, \infty]$ and $\mu_{t}:=\left(e_{t}\right)_{\#} \boldsymbol{\eta} \in \mathscr{P}(X)$ are representable as $u_{t} \mathfrak{m}$ with $u \in L_{t}^{\infty}\left(L_{x}^{p^{\prime}}\right)$. Then, the following two properties hold: 
(a) the family $\left(u_{t}\right)_{t \in(0, T)}$ is a weakly continuous solution to the the continuity equation;

(b) $\boldsymbol{\eta}$ is concentrated on $A C\left([0, T] ;\left(X, d_{\mathscr{A}^{*}}\right)\right)$, with

$$
|\dot{\eta}|(t) \leq\left|\boldsymbol{b}_{t}\right|(\eta(t)) \quad \text { for a.e. } t \in(0, T) \text {, for } \boldsymbol{\eta} \text {-a.e. } \eta \text {. }
$$

Remark 7.5. It is natural to conjecture that equality holds in (7.13), but presently we are not able to prove it, not even in the setting of RCD spaces: a notable exception is that of "gradient" derivations, see Theorem 9.5.

Arguing as in the last part of [AGS05, Thm. 8.3.1] one can prove that if in addition it holds $u \in L_{t}^{\infty}\left(L_{x}^{\infty}\right)$, then $\left(\mu_{t}\right)_{t}$ is an absolutely continuous curve in the Wasserstein space $W_{p}$ naturally associated to $d_{\mathscr{A} *}$ (see [GB14] for a deeper investigation of this connection in metric measure spaces).

Proof. We to integrate w.r.t. $\boldsymbol{\eta}$ the weak formulation

$$
\int_{0}^{t}-\psi^{\prime}(t) f \circ \eta(t) d t=\int_{0}^{T} \psi(t) d f\left(\boldsymbol{b}_{t}\right)(\eta(t)) d t
$$

with $f \in \mathscr{A}, \psi \in C_{c}^{1}(0, T)$, to recover the weak formulation of the continuity equation for $\left(u_{t}\right)$.

Given $f \in \mathscr{A}^{*}$, for $\boldsymbol{\eta}$-a.e. $\eta$, the map $t \mapsto f \circ \eta(t)$ is absolutely continuous, with

$$
f \circ \eta(t)-f \circ \eta(s)=\int_{s}^{t} d f\left(\boldsymbol{b}_{r}\right)(\eta(r)) d r, \quad \text { for all } s, t \in[0, T] .
$$

Since $\mathscr{A}^{*}$ is countable, there exists a Borel set $A$ of full $\mathscr{L}^{1} \otimes \mathfrak{m}$-measure such that, for every $f \in \mathscr{A}^{*}$, it holds $\left|d f\left(\boldsymbol{b}_{t}\right)\right|(x) \leq\left|\boldsymbol{b}_{t}\right|(x) \Gamma(f)^{1 / 2}(x)$ for $(t, x) \in A$. Moreover, using Fubini's theorem and the fact that the marginals of $\boldsymbol{\eta}$ are absolutely continuous w.r.t. $\mathfrak{m}$ we obtain that for $\boldsymbol{\eta}$-a.e. $\eta$ the set $\{t \in(0, T):(t, \eta(t)) \notin A\}$ is Lebesgue negligible. Thus, for $\boldsymbol{\eta}$-a.e. $\eta$ there holds

$$
\left|d f\left(\boldsymbol{b}_{t}\right)(\eta(t))\right| \leq\left|\boldsymbol{b}_{t}\right|(\eta(t)) \Gamma(f)^{1 / 2}(\eta(t)), \quad \text { for a.e. } t \in[0, T],
$$

and therefore

$$
|f \circ \eta(t)-f \circ \eta(s)| \leq \int_{s}^{t}\left|\boldsymbol{b}_{t}\right|(\eta(r)) \Gamma(f)^{1 / 2}(\eta(r)) d r, \quad \text { for all } s, t \in[0, T] .
$$

We conclude by taking the supremum over $f \in \mathscr{A}^{*}$.

Even though, as we explained in Remark 7.2, the (extended) distance is hidden in the choice of the family $\mathscr{A}^{*}$, we call the next result "superposition in metric measure spaces", because in most cases $\mathscr{A}^{*}$ consists precisely of distance functions from a countable dense set (see also the recent papers [Ba12] and [Sc13] for related results on the existence of suitable measures in the space of curves, and derivations).

Theorem 7.6 (Superposition principle in metric measure spaces). Assume (7.10), (7.11). Let $\boldsymbol{b}=\left(\boldsymbol{b}_{t}\right)_{t \in(0, T)}$ be a Borel family of derivations and let $\mu_{t}=u_{t} \mathfrak{m} \in \mathscr{P}(X), 0 \leq t \leq T$, be a weakly continuous solution to the continuity equation

$$
\partial_{t} \mu_{t}+\operatorname{div}\left(\boldsymbol{b}_{t} \mu_{t}\right)=0
$$


with

$$
u \in L_{t}^{\infty}\left(L_{x}^{p}\right), \quad \int_{0}^{T} \int\left|\boldsymbol{b}_{t}\right|^{r} d \mu_{t} d t<\infty, \quad \frac{1}{r}+\frac{1}{p} \leq 1 / 2 .
$$

Then there exists $\boldsymbol{\eta} \in \mathscr{P}(C([0, T] ; X))$ satisfying:

(a) $\boldsymbol{\eta}$ is concentrated on solutions $\eta$ to the $O D E \dot{\eta}=\boldsymbol{b}_{t}(\eta)$, according to Definition 7.3;

(b) $\mu_{t}=\left(e_{t}\right)_{\#} \boldsymbol{\eta}$ for any $t \in[0, T]$.

Proof. We enumerate by $f_{i}, i \geq 1$, the elements of $\mathscr{A}^{*}$ and define a continuous and injective $\operatorname{map} J: X \rightarrow \mathbb{R}^{\infty}$ by

$$
J(x):=\left(f_{1}(x), f_{2}(x), f_{3}(x), \ldots\right) .
$$

A simple consequence of (7.11), besides the injectivity we already observed, is that $J(X)$ is a closed subset of $\mathbb{R}^{\infty}$ and that $J^{-1}$ is continuous from $J(X)$ to $X$.

Defining $\nu_{t} \in \mathscr{P}\left(\mathbb{R}^{\infty}\right)$ by $\nu_{t}:=J_{\#} \mu_{t}, \boldsymbol{c}:(0, T) \times \mathbb{R}^{\infty} \rightarrow \mathbb{R}^{\infty}$ by

$$
c_{t}^{i}:= \begin{cases}\left(d f_{i}\left(\boldsymbol{b}_{t}\right)\right) \circ J^{-1} & \text { on } J(X) ; \\ 0 & \text { otherwise, }\end{cases}
$$

and noticing that

$$
\left|\boldsymbol{c}_{t}^{i}\right| \circ J \leq\left|\boldsymbol{b}_{t}\right|, \quad \mathfrak{m} \text {-a.e. in } X,
$$

the chain rule (see Proposition 3.2)

$$
d \phi\left(\boldsymbol{b}_{t}\right)(x)=\sum_{i=1}^{n} \frac{\partial \psi}{\partial z_{i}}\left(f_{1}(x), \ldots, f_{n}(x)\right) \boldsymbol{c}_{t}^{i}(x)
$$

for $\phi(x)=\psi\left(f_{1}(x), \ldots, f_{n}(x)\right)$ shows that the assumption of Theorem 7.1 are satisfied by $\nu_{t}$ with velocity $\boldsymbol{c}$, because (7.17) and $\mu_{t} \ll \mathfrak{m}$ give $\left|\boldsymbol{c}_{t}^{i}\right| \leq\left|\boldsymbol{b}_{t}\right| \circ J^{-1} \nu_{t}$-a.e. in $\mathbb{R}^{\infty}$.

As a consequence we can apply Theorem 7.1 to obtain $\boldsymbol{\lambda} \in \mathscr{P}\left(C\left([0, T] ; \mathbb{R}^{\infty}\right)\right)$ concentrated on solutions $\gamma \in A C\left([0, T] ; \mathbb{R}^{\infty}\right)$ to the $\mathrm{ODE} \dot{\gamma}=\boldsymbol{c}_{t}(\gamma)$ such that $\left(e_{t}\right)_{\#} \boldsymbol{\lambda}=\nu_{t}$ for all $t \in[0, T]$. Since all measures $\nu_{t}$ are concentrated on $J(X)$, there holds

$$
\gamma(t) \in J(X) \text { for } \boldsymbol{\lambda} \text {-a.e. } \gamma, \text { for all } t \in[0, T] \cap \mathbb{Q} \text {. }
$$

Then, the closedness of $J(X)$ and the continuity of $\gamma$ give $\gamma([0, T]) \subset J(X)$ for $\boldsymbol{\lambda}$-a.e. $\gamma$. For this reason, it makes sense to define

$$
\boldsymbol{\eta}:=\Theta_{\#} \boldsymbol{\lambda}
$$

where $\Theta: C([0, T] ; J(X)) \rightarrow C([0, T] ; X)$ is the map $\gamma \mapsto \Theta(\gamma):=J^{-1} \circ \gamma$. Since $\left(J^{-1}\right)_{\#} \nu_{t}=$ $\mu_{t}$, we obtain immediately that $\left(e_{t}\right)_{\#} \boldsymbol{\eta}=\mu_{t}$.

Let $i \geq 1$ be fixed. Since $f_{i} \circ \Theta(\gamma)=p_{i} \circ \gamma$, taking the definition of $\boldsymbol{c}_{i}$ into account we obtain that $f_{i} \circ \eta$ is absolutely continuous in $[0, T]$ and that

$$
\left(f_{i} \circ \eta\right)^{\prime}(t)=d f_{i}\left(\boldsymbol{b}_{t}\right)(\eta(t)) \text { a.e. in }(0, T) \text {, for } \boldsymbol{\eta} \text {-a.e. } \eta \text {. }
$$

We will complete the proof by showing that (7.18) extends from $\mathscr{A}^{*}$ to all of $\mathscr{A}$. By the chain rule we observe, first of all, that (7.18) extends from $f_{i}$ to smooth truncations of $f_{i}$. Therefore, by the density of $\mathscr{A}^{*}$ in $\mathbb{V}$, for any $f \in \mathscr{A}$ we can find $g_{n}$ satisfying: 
(a) $g_{n} \rightarrow f$ in $\mathbb{V}$ and $\left\|g_{n}\right\|_{\infty} \leq\|f\|_{\infty}+1$;

(b) $g_{n} \circ \eta \in A C([0, T])$ and $\left(g_{n} \circ \eta\right)^{\prime}(t)=d g_{n}\left(\boldsymbol{b}_{t}\right)(\eta(t))$ a.e. in $(0, T)$, for $\boldsymbol{\eta}$-a.e. $\eta$.

Since

$$
\iint_{0}^{T}\left|\left(f-g_{n}\right)(\eta(t))\right| d t d \boldsymbol{\eta}(\eta)=\int_{0}^{T} \int\left|f-g_{n}\right| u_{t} d \mathfrak{m} d t \rightarrow 0
$$

we can assume, possibly refining the sequence $\left(g_{n}\right)$, that $g_{n} \circ \eta \rightarrow f \circ \eta$ in $L^{1}(0, T)$ for $\boldsymbol{\eta}$-a.e. $\eta$.

In order to achieve Sobolev regularity of $f \circ \eta$ it remains to show convergence of the derivatives of $g_{n} \circ \eta$, namely $d g_{n}\left(\boldsymbol{b}_{t}\right)(\eta(t))$, to $d f\left(\boldsymbol{b}_{t}\right)(\eta(t))$. Arguing as in (7.19) we get

$$
\iint_{0}^{T}\left|d f\left(\boldsymbol{b}_{t}\right)(\eta(t))-d g_{n}\left(\boldsymbol{b}_{t}\right)(\eta(t))\right| d t d \boldsymbol{\eta}(\eta)=\int_{0}^{T} \int\left|d\left(f-g_{n}\right)\left(\boldsymbol{b}_{t}\right)\right| u_{t} d \mathfrak{m} d t \rightarrow 0
$$

because of (7.15) and the convergence $\Gamma\left(f-g_{n}\right) \rightarrow 0$ in $L^{1}(\mathfrak{m})$. Therefore, possibly refining once more $\left(g_{n}\right), d g_{n}(\boldsymbol{b})(\eta) \rightarrow d f(\boldsymbol{b})(\eta)$ in $L^{1}(0, T)$ for $\boldsymbol{\eta}$-a.e. $\eta$.

\section{Regular Lagrangian flows}

In this section we consider a Borel family of derivations $\boldsymbol{b}=\left(\boldsymbol{b}_{t}\right)_{t \in(0, T)}$ satisfying

$$
\boldsymbol{b} \in L_{t}^{1}\left(L_{x}^{1}+L_{x}^{\infty}\right)
$$

Under the assumption that the continuity equation has uniqueness of solutions in the class

$$
\mathcal{L}_{+}:=\left\{u \in L_{t}^{\infty}\left(L_{x}^{1} \cap L_{x}^{\infty}\right): t \mapsto u_{t} \text { is weakly continuous in }[0, T], u \geq 0\right\}
$$

for any initial datum $\bar{u} \in L^{1} \cap L^{\infty}(\mathfrak{m})$, and existence of solutions in the class

$$
\left\{u \in \mathcal{L}_{+}:\left\|u_{t}\right\|_{\infty} \leq C(\boldsymbol{b})\left\|u_{0}\right\|_{\infty} \forall t \in[0, T]\right\}
$$

for any nonnegative initial datum $\bar{u} \in L^{1} \cap L^{\infty}(\mathfrak{m})$, we prove existence and uniqueness of the regular flow $\boldsymbol{X}$ associated to $\boldsymbol{b}$. The concept of regular flow, adapted from [Amb04], is the following:

Definition 8.1 (Regular flows). We say that $\boldsymbol{X}:[0, T] \times X \rightarrow X$ is a regular flow (relative to $\boldsymbol{b})$ if the following two properties hold:

(i) $\boldsymbol{X}(0, x)=x$ and $\boldsymbol{X}(\cdot, x) \in C([0, T] ; X)$ for all $x \in X$;

(ii) for all $f \in \mathscr{A}, f(\boldsymbol{X}(\cdot, x)) \in W^{1,1}(0, T)$ and $\frac{d}{d t} f(\boldsymbol{X}(t, x))=d f\left(\boldsymbol{b}_{t}\right)(\boldsymbol{X}(t, x))$ for a.e. $t \in(0, T)$, for $\mathfrak{m}$-a.e. $x \in X$;

(iii) there exists a constant $C=C(\boldsymbol{X})$ satisfying $\boldsymbol{X}(t, \cdot)_{\#} \mathfrak{m} \leq C \mathfrak{m}$ for all $t \in[0, T]$.

Remark 8.2 (Invariance under modifications of $\boldsymbol{b}$ and $f$ ). Assume that $\boldsymbol{b}$ and $\tilde{\boldsymbol{b}}$ satisfy

$$
\text { for all } f \in \mathscr{A}, d f(\boldsymbol{b})=d f(\tilde{\boldsymbol{b}}) \mathscr{L}^{1} \otimes \mathfrak{m} \text {-a.e. in }(0, T) \times X \text {. }
$$


Then $\boldsymbol{X}$ is a regular flow relative to $\boldsymbol{b}$ if and only if $\boldsymbol{X}$ is a regular flow relative to $\tilde{\boldsymbol{b}}$. Indeed, let us fix $f \in \mathscr{A}$ and let us notice that for all $t \in(0, T)$ such that $\mathfrak{m}\left(\left\{d f\left(\boldsymbol{b}_{t}\right) \neq d f\left(\tilde{\boldsymbol{b}}_{t}\right)\right\}\right)=0$, condition (iii) of Definition 8.1 gives

$$
d f\left(\boldsymbol{b}_{t}\right)(\boldsymbol{X}(t, x))=d f\left(\tilde{\boldsymbol{b}}_{t}\right)(\boldsymbol{X}(t, x))=0, \quad \text { for } \mathfrak{m} \text {-a.e. } x \in X .
$$

Thanks to (8.4) and Fubini's theorem, the condition $\mathfrak{m}\left(\left\{d f\left(\boldsymbol{b}_{t}\right) \neq d f\left(\tilde{\boldsymbol{b}}_{t}\right)\right\}\right)=0$ is satisfied for a.e. $t \in(0, T)$. Hence, we may apply once more Fubini's theorem to get

$$
d f\left(\boldsymbol{b}_{t}\right)(\boldsymbol{X}(t, x))=d f\left(\tilde{\boldsymbol{b}}_{t}\right)(\boldsymbol{X}(t, x))=0 \quad \text { a.e. in }(0, T), \quad \text { for } \mathfrak{m} \text {-a.e. } x \in X .
$$

With a similar argument, one can show that if we modify not only $d f(\boldsymbol{b})$, but also $f$ in a $\mathfrak{m}$-negligible set, to obtain a Borel representative $\tilde{f}$, then $f(\boldsymbol{X}(\cdot, x)) \in W^{1,1}(0, T)$ and $\frac{d}{d t} f(\boldsymbol{X}(t, x))=\boldsymbol{b}_{t}(\boldsymbol{X}(t, x))$ for a.e. $t \in(0, T)$ if and only if $\tilde{f}(\boldsymbol{X}(\cdot, x)) \in W^{1,1}(0, T)$ and $\frac{d}{d t} \tilde{f}(\boldsymbol{X}(t, x))=\boldsymbol{b}_{t}(\boldsymbol{X}(t, x))$ for a.e. $t \in(0, T)$, because Fubini's theorem gives $\tilde{f}(\boldsymbol{X}(t, x))=$ $f(\boldsymbol{X}(t, x))$ for a.e. $t \in(0, T)$, for $\mathfrak{m}$-a.e. $x \in X$. For this reason the choice of a Borel representative of $f \in \mathscr{A}$ is not really important. Whenever this is possible, the natural choice of course is given by the continuous representative.

The main result of the section is the following existence and uniqueness result. We stress that uniqueness is understood in the pathwise sense, namely $\boldsymbol{X}(\cdot, x)=\boldsymbol{Y}(\cdot, x)$ in $[0, T]$ for m-a.e. $x \in X$, whenever $\boldsymbol{X}$ and $\boldsymbol{Y}$ are regular Lagrangian flows relative to $\boldsymbol{b}$.

Theorem 8.3 (Existence and uniqueness of the regular Lagrangian flow). Assume (8.1), and that the continuity equation induced by $\boldsymbol{b}$ has uniqueness of solutions in $\mathcal{L}_{+}$for all initial datum $\bar{u} \in L^{1} \cap L^{\infty}(\mathfrak{m})$, as well as existence of solutions in the class (8.3) for all nonnegative initial datum $\bar{u} \in L^{1} \cap L^{\infty}(\mathfrak{m})$. Then there exists a unique regular Lagrangian flow relative to b.

Proof. Let $B \in \mathscr{B}(X)$ with positive and finite $\mathfrak{m}$-measure and let us build first a "generalized" flow starting from $B$. To this aim, we take $\bar{u}=\chi_{B} / \mathfrak{m}(B)$ as initial datum and we apply first the assumption on existence of a solution $u \in \mathcal{L}_{+}$starting from $\bar{u}$, with $u_{t} \leq C(\boldsymbol{b}) / \mathfrak{m}(B)$, and then the superposition principle stated in Theorem 7.6 to obtain $\boldsymbol{\eta} \in \mathscr{P}(C([0, T] ; X))$ whose time marginals are $u_{t} \mathfrak{m}$, concentrated on solutions to the ODE $\dot{\eta}=\boldsymbol{b}_{t}(\eta)$. Then, Theorem 8.4 below (which uses the uniqueness part of our assumptions relative to the continuity equation) provides a representation

$$
\boldsymbol{\eta}=\frac{1}{\mathfrak{m}(B)} \int_{B} \delta_{\eta_{x}} d \mathfrak{m}(x) .
$$

Setting $\boldsymbol{X}(\cdot, x)=\eta_{x}(\cdot)$ for $x \in B$, it follows that $\boldsymbol{X}: B \times[0, T]$ is a regular flow, relative to $\boldsymbol{b}$, with the only difference that (i) and (ii) in Definition 8.1 have to be understood for $\mathfrak{m}$-a.e. $x \in B$, and

$$
\boldsymbol{X}(t, \cdot)_{\#}(\bar{u} \mathfrak{m})=\left(e_{t}\right)_{\#} \boldsymbol{\eta}=u_{t} \mathfrak{m} \leq \frac{C(\boldsymbol{b})}{\mathfrak{m}(B)} \mathfrak{m} .
$$

Next we prove consistency of these "local" flows $\boldsymbol{X}_{B}$. If $B_{1} \subset B_{2}$ with $\mathfrak{m}\left(B_{1}\right)>0$ and $\mathfrak{m}\left(B_{2}\right)<\infty$, we can consider the measure

$$
\boldsymbol{\eta}:=\frac{1}{2 \mathfrak{m}\left(B_{1}\right)} \int_{B_{1}}\left(\delta_{\boldsymbol{X}_{B_{1}}(\cdot, x)}+\delta_{\boldsymbol{X}_{B_{2}}(\cdot, x)}\right) d \mathfrak{m}(x) \in \mathscr{P}(C([0, T] ; X))
$$


to obtain from Theorem 8.4 that $\boldsymbol{X}_{B_{1}}(\cdot, x)=\boldsymbol{X}_{B_{2}}(\cdot, x)$ for m-a.e. $x \in B_{1}$.

Having gained consistency, we can build a regular Lagrangian flow by considering a nondecreasing sequence of a Borel sets $B_{n}$ with positive and finite $\mathfrak{m}$-measure whose union covers $\mathfrak{m}$-almost all of $X$ and the corresponding local flows $\boldsymbol{X}_{n}: B_{n} \times[0, T] \rightarrow X$. Notice that we needed a quantitative upper bound on $\boldsymbol{X}_{n}(t, \cdot)_{\#}\left(\chi_{B_{n}} \mathfrak{m}\right)$ precisely in order to be able to pass to the limit in condition (iii) of Definition 8.1, since (8.5) gives $\boldsymbol{X}(t, \cdot)_{\#}\left(\chi_{B} \mathfrak{m}\right) \leq C(\boldsymbol{b}) \mathfrak{m}$.

This completes the existence part. The uniqueness part can be proved using once more Theorem 8.4 and the same argument used to show consistency of the "local" flows.

Theorem 8.4 (No splitting criterion). Assume (8.1), and that the continuity equation induced by $\boldsymbol{b}$ has at most one solution in $\mathcal{L}_{+}$for all $\bar{u} \in L^{1} \cap L^{\infty}(\mathfrak{m})$. Let $\boldsymbol{\eta} \in \mathscr{P}(C([0, T] ; X))$ be satisfying:

(i) $\boldsymbol{\eta}$ is concentrated on solutions $\eta$ to the $O D E \dot{\eta}=\boldsymbol{b}_{t}(\eta)$;

(ii) there exists $L_{0} \in[0, \infty)$ satisfying

$$
\left(e_{t}\right)_{\#} \boldsymbol{\eta} \leq L_{0} \mathfrak{m} \quad \forall t \in[0, T] .
$$

Then the conditional measures $\boldsymbol{\eta}_{x} \in \mathscr{P}(C([0, T] ; X))$ induced by the map $e_{0}$ are Dirac masses for $\left(e_{0}\right)_{\#} \boldsymbol{\eta}$-a.e. $x$; equivalently, there exist $\eta_{x} \in C([0, T] ; X)$ such that $\eta(0)=x$ and solving the $O D E \dot{\eta}=\boldsymbol{b}_{t}(\eta)$, satisfying $\boldsymbol{\eta}=\int \delta_{\eta_{x}} d\left(e_{0}\right)_{\#} \boldsymbol{\eta}(x)$.

Proof. Using the uniqueness assumption at the level of the continuity equation, as well as the implication provided by Lemma 7.4, the decomposition procedure of [AC08, Thm. 18] (that slightly improves the original argument of [Amb04, Thm. 5.4], where comparison principle for the continuity equation was assumed) gives the result.

\section{$9 \quad$ Examples}

In this section, on one hand we illustrate relevant classes of metric measure spaces for which our abstract theory applies. On the other hand we try to compare our results on the wellposedness of the continuity equation with the ones obtained in other papers, for particular classes of spaces. Several variants of the existence and uniqueness results are possible, varying the regularity and the growth conditions imposed on $\boldsymbol{b}$ and on the density $u_{t}$; we focus mainly on the issue of uniqueness, since existence in particular class of spaces (e.g. the Euclidean ones) can be often be obtained by ad hoc methods (e.g. convolution of the components of the vector field, which preserve bounds on divergence) not available in general spaces. Also, we will not discuss the existence/uniqueness of the flow, which follow automatically from well-posedness at the PDE level using the transfer mechanisms presented in Section 8. We list the examples following, to some extent, chronological order and level of complexity.

\subsection{Euclidean spaces: the DiPerna-Lions theory}

The theory of well posedness for flows and for transport and continuity equations was initiated by DiPerna-Lions in [DL89] and it (quite obviously) fits into our abstract setting. More explicitly we let, in the basic setup (2.1), $X=\mathbb{R}^{n}, \mathfrak{m}=\mathscr{L}^{n}$ the Lebesgue measure and

$$
\mathcal{E}(f)=\int|\nabla f|^{2}(x) d \mathscr{L}^{n}(x), \quad \text { for } f \in W^{1,2}\left(\mathbb{R}^{n}\right),
$$


so that $\Delta$ is the usual Laplacian and $\left(\mathrm{P}_{t}\right)_{t}$ is the heat semigroup, that corresponds (up to a factor 2 in the time scale) to the transition semigroup of the Brownian motion, which is conservative. The algebra $\mathscr{A}$ of Section 2.3 can be chosen to be the space of Lipschitz functions with compact support.

Given a Borel vector field $b=\sum_{i=1}^{n} b^{i} e_{i}$, with $b \in\left(L^{1}+L^{\infty}\right)^{n}$, its associated derivation $\boldsymbol{b}$ is

$$
\mathscr{A} \ni f \mapsto d f(\boldsymbol{b})=\sum_{i=1}^{n} b_{i} \frac{\partial f}{\partial x^{i}} .
$$

Obviously, $\operatorname{div} \boldsymbol{b}$ is the usual distributional divergence and $D^{s y m} \boldsymbol{b}$ is the symmetric part of the distributional derivative of $\boldsymbol{b}$. Then, the uniqueness Theorem 5.4 above corresponds to [DL89, Corollary II.1], as long as $q \in(1, \infty]$.

On the other hand, in Euclidean spaces the strong local convergence of commutators depends on local regularity assumptions on $\boldsymbol{b}$ (and the use of convolutions with compact support), while our setting is intrinsically global. In order to adapt our methods to this case, one could "localize the Dirichlet form" by considering $X=B_{r}(0)$ and the form

$$
\mathcal{E}_{r}(f)=\int_{B_{r}}|\nabla f|^{2} d \mathscr{L}^{n}, \quad \text { for } f \in H^{1}\left(B_{r}\right) .
$$

Thus $\Delta$ would be the Laplacian with Neumann boundary conditions and $\left(\mathrm{P}_{t}\right)_{t}$ would be the semigroup correspondent to the Brownian motion reflected at the boundary $\partial B_{r}(0)$, which is still conservative. Being the ball convex, it can be proved that $\mathrm{BE}_{2}(0, \infty)$ (and even $\mathrm{BE}(0, n)$ ) still holds, see for instance [AGS11b, Thm. 6.20].

A second major difference is that uniqueness assuming the regularity $b \in\left(W^{1,1}\right)^{n}$ (or even $b \in(B V)^{n}$, the case considered in [Amb04]) is not covered. Indeed, the $B V$ case seems difficult to reach in the abstract setting, due to the present lack of a covariant derivative (but see [Gig14]).

\subsection{Weighted Riemannian manifolds}

Our arguments extend the classical DiPerna-Lions theory to the setting of weighted Riemannian manifolds. Of course, in order to prove strong convergence of commutators and the fact that solutions are renormalized one can always argue by local charts, but computations become more cumbersome, compared to the Euclidean case, and here the advantages of our intrinsic approach become more manifest.

Let $(M, \boldsymbol{g})$ be a smooth Riemannian manifold and let $\mu$ be its associated Riemannian volume measure. Assume that the Ricci curvature tensor $\mathrm{Ric}_{\boldsymbol{g}}$ is pointwise bounded from below (in the sense of quadratic form) by some constant $K \in \mathbb{R}$. More generally, one can add a "weight" $V: M \rightarrow \mathbb{R}$ to the measure, i.e. consider a smooth non-negative function and assume that the Bakry-Émery curvature tensor is bounded from below by $K \in \mathbb{R}$, i.e.

$$
\operatorname{Ric}_{\boldsymbol{g}}+\operatorname{Hess}(V) \geq K \text {. }
$$

The form (on smooth compactly supported functions)

$$
f \mapsto \mathcal{E}_{V}(f)=\int_{M} \boldsymbol{g}(\nabla f, \nabla f) e^{-V} d \mu
$$


is closable and we are in the setup (2.1). Once more, the algebra $\mathscr{A}$ of Section 2.3 can be chosen to be the space of Lipschitz functions with compact support.

When $V=0$, Bochner's formula entails that $\mathrm{BE}_{2}(K, \infty)$ holds and it is a classical result due to S.-T. Yau that the heat semigroup is conservative. In the case of weighted measures, analogous results can be found in [Bak94b, Prop. 6.2] for the curvature bound and in [Gri99, Thm. 9.1] for the conservativity of $\mathrm{P}$, relying on a correspondent volume comparison theorem, see e.g. [WW09, Thm. 1.2].

Given a Borel vector field $b$, i.e. a Borel section of the tangent bundle of $M$, its associated derivation $\boldsymbol{b}$ acts on smooth functions by

$$
f \mapsto d f(\boldsymbol{b})=\boldsymbol{g}(b, \nabla f) .
$$

The divergence can be given in terms of the $\mu$-distributional divergence of $b$ by

$$
\operatorname{div} \boldsymbol{b}=\operatorname{div} b-\boldsymbol{g}(\boldsymbol{b}, \nabla V),
$$

while the deformation is the symmetric part of the distributional covariant derivative, see Remark 5.3.

\subsection{Abstract Wiener spaces}

Let $(X, \gamma, \mathcal{H})$ be an abstract Wiener space, i.e. $X$ is a separable Banach space, $\gamma$ is a centered non-degenerate Gaussian measure on $X$, with covariance operator $Q: X^{*} \mapsto X$ and $\mathcal{H} \subset X$ is its associated Cameron-Martin space, which is naturally endowed with a Hilbertian norm. Moreover, $Q X^{*} \subset \mathcal{H}$.

We define the set of smooth cylindrical functions $\mathcal{F C}_{b}^{\infty}(X)$ as the set of all functions $f(x)$ representable as $\varphi\left(x_{1}^{*}(x), \ldots, x_{n}^{*}(x)\right)$, with $\varphi: \mathbb{R}^{n} \rightarrow \mathbb{R}$ smooth and bounded, $x_{i}^{*} \in X^{*}$ for $i \in\{1, \ldots, n\}$, for some integer $n \geq 1$.

We introduce a notion of "gradient" on functions $f \in \mathcal{F C}_{b}^{\infty}(X)$ letting $\nabla_{H} f=Q d f$, where $d f$ is the Frechét differential of $f$. With these definitions, for $f=\varphi\left(x_{1}^{*}, \ldots, x_{n}^{*}\right)$, there holds

$$
\nabla_{\mathcal{H}} f(x)=\sum_{j=1}^{n} \frac{\partial \varphi}{\partial z_{j}} Q x_{j}^{*}=\sum_{i} \frac{\partial f}{\partial h_{i}}(x) h_{i}, \quad \text { where } \quad \frac{\partial f}{\partial h_{i}}(x)=\lim _{\varepsilon \rightarrow 0} \frac{f\left(x+\varepsilon h_{i}\right)-f(x)}{\varepsilon}
$$

where $\left(h_{i}\right)$ is any orthonormal basis of $\mathcal{H}$.

It is well-known [BH91] that Sobolev-Malliavin calculus on $(X, \gamma, \mathcal{H})$ fits into the setting (2.1), considering the closure of the quadratic form

$$
\mathcal{E}(f)=\int_{X}\left|\nabla_{\mathcal{H}} f\right|_{\mathcal{H}}^{2} d \gamma, \quad \text { for every } f \in \mathcal{F C}_{b}^{\infty}(X)
$$

The domain $\mathbb{V}$ coincides with the space $W^{1,2}(X, \gamma)$. The semigroup $\mathrm{P}$ is the OrnsteinUhlenbeck semigroup, given by Mehler's formula

$$
\mathrm{P}_{t} f(x)=\int_{X} f\left(e^{-t} x+\sqrt{1-e^{-2 t}} y\right) d \gamma(y), \quad \text { for } \gamma \text {-a.e. } x \in X \text {. }
$$

From this expression, it is easy to show that $\mathrm{BE}_{2}(1, \infty)$ holds (indeed, on cylindrical functions $\nabla \mathrm{P}_{t} f=e^{-t} \mathrm{P}_{t} \nabla f$, understanding the action of the semigroup componentwise); it is a classical 
result that $\mathcal{E}$ is quasi-regular, see e.g. [Bog98, Thm. 5.9.9]. We let $\mathscr{A}=\mathcal{F C}_{b}^{\infty}(X)$, which is well-known to be dense in every $L^{p}$-space and satisfy $(2.20)$ by Mehler's formula above: in particular we obtain density in $\mathbb{V}_{p}$ spaces by Lemma 5.6 and Lemma 2.2.

Given an $\mathcal{H}$-valued field $b=\sum_{i} b^{i} h_{i}$, we introduce the derivation $f \mapsto \boldsymbol{b}(f)=\sum_{i} b^{i} \frac{\partial f}{\partial h_{i}}$ and we briefly compare our well-posedness results for the continuity equation with those contained in [AF09]. Combining Proposition 6.8 and the subsequent remark, we obtain that our notion of deformation for $\boldsymbol{b}$ is comparable to that of $(\nabla b)^{\text {sym }}$ introduced in [AF09, Def. 2.6]. Precisely, it can be proved that if $b \in L D^{q}(\gamma ; \mathcal{H})$ for some $q>1$, then the deformation of $\boldsymbol{b}$ is of type $(r, s)$, for any $r, s$, with $q^{-1}+r^{-1}+s^{-1}=1$. It is then easy to realize that Theorem 5.4 entails the uniqueness part of [AF09, Thm. 3.1], with the exception, as we observed in connection to the Euclidean theory, of the case $b \in W^{1,1}(X, \gamma ; H)$ (the case $b \in B V(X, \gamma ; H)$ has been recently settled in [Tre13]).

\subsection{Gaussian Hilbert spaces}

We let $X=H$ be a separable Hilbert space, with norm $|\cdot|$, in the setting introduced in the previous section, namely $\gamma$ is a Gaussian centered and nondegenerate measure in $H$. By identifying $H=H^{*}$ via the Riesz isomorphism induced by the norm, the covariance operator $Q: H \rightarrow H$ is a symmetric positive trace class operator, thus compact. In this setting the Cameron-Martin space is $\mathcal{H}=Q^{1 / 2} H$, with the norm $|h|_{\mathcal{H}}=\left|Q^{-1 / 2} h\right|$.

We let $\left(e_{i}\right) \subset H$ be an orthonormal basis of $H$ consisting of eigenvectors of $Q$, with eigenvalues $\left(\lambda_{i}\right)$, i.e. $Q e_{i}=\lambda_{i} e_{i}$ for every $i \geq 1$ : in this setting, we define the class of smooth cylindrical functions $\mathcal{F C}_{b}^{\infty}(H)$ as those functions $f: X \rightarrow \mathbb{R}$ of the form $f(x)=$ $\varphi\left(\left\langle e_{i}, x\right\rangle, \ldots\left\langle e_{n}, x\right\rangle\right)$, with $\varphi: \mathbb{R}^{n} \rightarrow \mathbb{R}$ smooth and bounded. Given $f \in \mathcal{F C}_{b}^{\infty}(H)$, from its Fréchet derivative $d f: H \mapsto H^{*}$ we introduce $\nabla f: H \mapsto H$ via $H=H^{*}$, in coordinates:

$$
\nabla f(x)=\sum_{i} \partial_{i} f(x) e_{i}, \quad \text { where } \partial_{i} f(x)=\lim _{\varepsilon \rightarrow 0} \frac{f\left(x+\varepsilon e_{i}\right)-f(x)}{\varepsilon} .
$$

To recover the abstract setting of the previous section, notice that family $h_{i}=\lambda_{i}^{1 / 2} e_{i}$ is an orthonormal basis of $\mathcal{H}$ and that $\partial / \partial h_{i}=\lambda_{i}^{-1 / 2} \partial_{i}$, thus it holds $Q \nabla f=\nabla_{\mathcal{H}} f$.

For $\alpha \in \mathbb{R}$, we introduce the form

$$
\mathcal{E}^{\alpha}(f)=\int_{X}\left|Q^{(1-\alpha) / 2} \nabla f\right|^{2} d \gamma, \quad f \in \mathcal{F C}_{b}^{\infty}(H),
$$

which is closable: its domain is the space $W_{\alpha}^{1,2}(H, \gamma)$, see [DP04, Chapters 1 and 2] for more details. Evidently, we recover $(2.1)$, with $\Gamma(f)=\sum_{i} \lambda_{i}^{1-\alpha}\left|\partial_{i} f\right|^{2}$. Notice that the associate distance is the one induced by the norm $\left|Q^{(\alpha-1) / 2} x\right|$, which is extended if and only if $\alpha<1$.

The associated semigroup can be still be seen as the transition semigroup of an infinite dimensional SDE, and its infinitesimal generator $\Delta_{\alpha}$ is given by

$$
\Delta_{\alpha} f(x)=\operatorname{Tr}\left[Q^{1-\alpha} D^{2} f(x)\right]-\left\langle x, Q^{-\alpha} \nabla f(x)\right\rangle, \quad f \in \mathcal{F C}_{b}^{\infty}(H) .
$$

It can be shown that $\mathrm{BE}_{2}(1, \infty)$ holds [DP04, Prop. 2.60]. We let $\mathscr{A}=\mathcal{F C}_{b}^{\infty}(H)$, which is dense in every $L^{p}(\mathfrak{m})$ space and satisfies $(2.20)$, thus obtaining density results in $\mathbb{V}_{p}(p \in[1, \infty))$ by Lemma 5.6 and Lemma 2.2. 
For $\alpha=0$, we recover the abstract Wiener space setting discussed above, while for $\alpha=1$ we obtain the setting of [DFR13]. We show that our results encompass those in [DFR13] and analogues hold for any $\alpha \in \mathbb{R}$.

Given $b: H \mapsto H, b=\sum_{i} b_{i} e_{i}$ Borel, we consider the map

$$
\mathcal{F e}_{b}^{\infty}(H) \ni f \mapsto d f(\boldsymbol{b}):=\langle b, \nabla f\rangle_{H}=\sum_{i} b_{i} \partial_{i} f .
$$

If $\left|Q^{(\alpha-1) / 2} b\right| \in L^{q}(H, \gamma)$ for some $q \in[1, \infty]$, then $\boldsymbol{b}$ is a well-defined derivation, with $|\boldsymbol{b}| \leq$ $\left|Q^{(\alpha-1) / 2} b\right|$.

The Cameron-Martin theorem entails an integration by parts formula [DP04, Thm. 1.4 and Lemma 1.5] that reads in our notation as

$$
\operatorname{div} \boldsymbol{e}_{i}(x)=-\frac{\left\langle e_{i}, x\right\rangle}{\lambda_{i}}, \quad \text { where } d f\left(\boldsymbol{e}_{i}\right)=\partial_{i} f
$$

On smooth "cylindrical" fields $b=\sum_{i}^{n} b_{i} e_{i}$, this gives

$$
\operatorname{div} \boldsymbol{b}(x)=\sum_{i} \partial_{i} b_{i}(x)-\frac{\left\langle e_{i}, x\right\rangle}{\lambda_{i}} b_{i}
$$

where the series reduces to a finite sum. Notice that the expression does not depend on $\alpha$ but only on $\gamma$, in agreement with the notion of divergence as dual to derivation.

Notice also that the boundedness of the Gaussian Riesz transform [Bog98, Prop. 5.88] entails that if $\boldsymbol{b} \in W^{1, p}(H, \gamma, \mathcal{H})$, then $\operatorname{div} \boldsymbol{b} \in L^{p}(H, \gamma)$. These are only sufficient conditions and their assumptions would force us to limit the discussion to $\mathcal{H}$-valued fields, as in [DFR13] (see Section 5 therein). Our results hold even for some classes of fields not taking values in $\mathcal{H}$, see at the end of this section.

Arguing on smooth cylindrical functions,

$$
\int D^{s y m} \boldsymbol{b}(f, g) d \gamma=\int \sum_{i, j} \frac{1}{2}\left[\left(\frac{\lambda_{i}}{\lambda_{j}}\right)^{(1-\alpha) / 2} \partial_{i} b_{j}+\left(\frac{\lambda_{j}}{\lambda_{i}}\right)^{(1-\alpha) / 2} \partial_{j} b_{i}\right]\left(\lambda_{i}^{(1-\alpha) / 2} \partial_{i} f\right)\left(\lambda_{j}^{(1-\alpha) / 2} \partial_{j} f\right) d \gamma
$$

thus our bound on $D^{\text {sym }} \boldsymbol{b}$ is implied by an $L^{q}$ bound of the Hilbert-Schmidt norm of the expression is square brackets above (a fact that could also be seen as a consequence of Proposition 6.8 and the subsequent remark, setting $V_{i}(x)=\lambda_{i}^{(\alpha-1) / 2}\left\langle e_{i}, x\right\rangle$, for $\left.i \geq 1\right)$.

Comparing our setting with that in [DFR13], it is clear that Theorem 2.3 therein is a consequence of Theorem 5.4.

We end this section considering a field $b$ taking values outside $\mathcal{H}$, to which our theory applies (although well-posedness was already shown in [MWZ05]). Assume that that each eigenvalue of $Q$ admits a two-dimensional eigenspace thus, slightly changing the notation, we write $\left(e_{i}, \tilde{e}_{i}\right)$ for an orthonormal basis of $H$ consisting of eigenvectors of $Q$. We let

$$
b=\sum_{i=1}^{\infty} \lambda_{i}^{1 / 2}\left[\left(\operatorname{div} \tilde{\boldsymbol{e}}_{i}\right) e_{i}-\left(\operatorname{div} \boldsymbol{e}_{i}\right) \tilde{e}_{i}\right], \text { thus } \int\left|Q^{(\alpha-1) / 2} b\right|^{2} d \gamma=\sum_{i=1}^{\infty} \lambda_{i}^{\alpha} .
$$

The series above converges if $\alpha=1$, and it does not if $\alpha=0$. Since $\left(\operatorname{div} \boldsymbol{e}_{i}, \operatorname{div} \tilde{\boldsymbol{e}}_{i}\right)_{i}$ are independent, Kolmogorov's 0-1 law entails that $b$ is well defined as an $H$-valued map, but $b(x) \notin \mathcal{H}$ for $\gamma$-a.e. $x \in H$. The derivation $\boldsymbol{b}$ is therefore well-defined if $\alpha=1$, and $|\boldsymbol{b}| \in L^{2}(\mathfrak{m})$. From its structure and (9.1), both its divergence and its deformation are seen to be identically 0 , thus our results apply. 


\subsection{Log-concave measures}

Let $(H,|\cdot|)$ be a separable Hilbert space and let $\gamma$ be a log-concave probability measure on $H$, i.e. for all open sets $B, C \subset H$,

$$
\log \gamma((1-t) B+t C) \geq(1-t) \log \gamma(B)+t \log \gamma(C), \text { for every } t \in[0,1] .
$$

Assume also that $\gamma$ is non-degenerate, namely that it is not concentrated on a proper closed subspace of $H$. Consider the quadratic form

$$
\mathcal{E}_{\gamma}(f)=\int|\nabla f|^{2} d \gamma, \quad \text { defined for } f \in C_{b}^{1}(H),
$$

where $C_{b}^{1}(H)$ denotes the space of continuously Fréchet differentiable functions which are bounded together with their differential.

It is shown in [ASZ09] that the $\mathcal{E}_{\gamma}$ is closable, extending previous results obtained under more restrictive assumptions on $\gamma$. Actually, since in [ASZ09] the so-called EVI property for the associated semigroup $P$ is proved, and since in [AGS11b] this is proved to be one of the equivalent characterizations of $\operatorname{RCD}$, it follows that $(H,|\cdot|, \gamma)$ is an $\operatorname{RCD}(0, \infty)$ space, thus the results in the next Section 9.6 apply and we already obtain abstract well-posedness result under no additional assumption on $\gamma$. Recall that in that abstract setting $\mathscr{A}$ can be takes as the space of Lipschitz functions with bounded support.

Let $\left(e_{i}\right)_{i \geq 1} \subset H$ be an orthonormal basis. For every $f \in \mathbb{V}$, there exist $f_{n} \in C_{b}^{1}(H)$ such that $f_{n} \rightarrow f$ in $L^{2}(\gamma)$ and

$$
\lim _{n, m \rightarrow \infty} \mathcal{E}_{\gamma}\left(f_{n}-f_{m}\right) \rightarrow 0
$$

thus an $H$-valued "gradient" $\nabla f=\sum_{i} \partial_{i} f e_{i}$ is $\gamma$-a.e. defined in $H$.

Let $b: H \mapsto H, b=\sum_{i} b_{i} e_{i}$ : we associate the derivation $f \mapsto d f(\boldsymbol{b})=\sum_{i} b_{i} f_{i}$, thus $|\boldsymbol{b}| \leq|b|$. For $v \in H$, we write $\boldsymbol{v}$ for the constant derivation corresponding to the constant vector field equal to $v$, and $\boldsymbol{e}_{i}$ for the derivation corresponding to $e_{i}$.

Let us remark that, in this very general setting setting, bounds on the divergence of a given field $\boldsymbol{b}$ seem to be difficult to obtain, even for constant vector fields: this is due to the fact that presently it is not known whether every log-concave measure $\gamma$ admits at least one nonzero direction $v$ such that $\operatorname{div} \boldsymbol{v} \in L^{1}(\mathfrak{m}),[\operatorname{Bog} 10, \S 4.3]$. On the other hand, our abstract arguments do not require any absolute continuity of $\gamma$ with respect to a Gaussian or other product measures and combining our abstract well-posedness results with Theorem 6.7, we are able to provide non-trivial derivations that admit a well-posed flow, e.g. gradient derivations of functions in $D_{L^{4}}(\Delta)$, such as those of the form $\int_{1 / 2}^{1} \mathrm{P}_{t} f d t$, for $f \in L^{4}(\mathfrak{m})$.

To state an explicit sufficient condition to bound the deformation of $\boldsymbol{b}$, we assume that $\operatorname{div} \boldsymbol{e}_{i} \ll \mathfrak{m}$ and, denoting by $\beta_{i}$ the density, we require that, for $i \geq 1, \beta_{i} \in \mathbb{V}$ or equivalently that the function $x \mapsto V_{i}(x)=\left\langle e_{i}, x\right\rangle$ satisfies $\Delta V_{i} \in \mathbb{V}$, thus Proposition 6.8 gives $\left\|D^{s y m} \boldsymbol{b}\right\|_{r, s}<\infty$ if $\left[\partial_{i} b_{j}+\partial_{j} b_{i}\right]_{i, j} \in L^{q}\left(\gamma ; \ell^{2}(\mathbb{N} \otimes \mathbb{N})\right)$, provided that $r, s \geq 4$.

We conclude by comparing our results in this setting with [KR13, Thm. 7.6], where uniqueness for the continuity equation is obtained in the case of log-concave measures formally given by $\gamma=$ " $e^{-V} d \mathscr{L}^{\infty}$ ", for convex Hamiltonians $V$ of specific form. In particular, the assumptions on $\beta_{i}$ imposed therein are stronger than ours. Their assumptions on the field $b$ in [KR13] entail that $|\boldsymbol{b}| \in L^{a_{1}}(\gamma)$, for some $a_{1}>1$ and that $\left[\partial_{i} b_{j}+\partial_{j} b_{i}\right]_{i, j} \in L^{a_{2}}\left(\gamma ; \ell^{2}(\mathbb{N} \otimes \mathbb{N})\right)$, for some $a_{2}>4$. Moreover, to deduce uniqueness, $\operatorname{div} \boldsymbol{b} \in L^{q}(\gamma)$, for some $q>1$ is also assumed. Therefore, if $a_{1} \geq 2$ and $q \geq 2$, we are in a position to recover, via Proposition 6.8, such a uniqueness result as a special case of Theorem 5.4. 


\section{6 $\operatorname{RCD}(K, \infty)$ metric measure spaces}

Recall that the class $\mathrm{CD}(K, \infty)$, introduced and deeply studied in [LV09], [Stu06a], [Stu06b] consists of complete metric measure spaces such that the Shannon relative entropy w.r.t. $\mathfrak{m}$ is $K$-convex along Wasserstein geodesics, see [Vil09] for a full account of the theory and its geometric and functional implications. The class of $\operatorname{RCD}(K, \infty)$ metric measure spaces was first introduced in [AGS11b], from a metric perspective, as class of spaces smaller than that of $\mathrm{CD}(K, \infty)$ metric measure spaces. The additional requirement, in this class of spaces, is that the so-called Cheeger energy is quadratic; with this axiom, Finsler geometries are ruled out and stronger structural (and stability) properties can be estabilished. Subsequently, connections with the theory of Dirichlet forms gave rise to a series of works, [AGS12], [Sav13] and [AMS13]. For a brief introduction to the setting and its notation, we refer to Sections 4.1 and 4.2 in [Sav13], and in particular to Theorem 4.1 therein, which collects non-trivial equivalences among different conditions.

We will use the notation $W^{1,2}(X, d, \mathfrak{m})$ for the Sobolev space, Ch for the Cheeger energy arising from the relaxation in $L^{2}(X, \mathfrak{m})$ of the local Lipschitz constant

$$
|\mathrm{D} f|(x):=\limsup _{y \rightarrow x} \frac{|f(y)-f(x)|}{d(y, x)}
$$

of $L^{2}(\mathfrak{m})$ and Lipschitz maps $f: X \rightarrow \mathbb{R}$.

To introduce $\operatorname{RCD}(K, \infty)$ spaces we restrict the discussion to metric measure spaces $(X, d, \mathfrak{m})$ satisfying the following three conditions:

(a) $(X, d)$ is a complete and separable length space;

(b) $\mathfrak{m}$ is a nonnegative Borel measure with $\operatorname{supp}(\mathfrak{m})=X$, satisfying

$$
\mathfrak{m}\left(B_{r}(x)\right) \leq c \mathrm{e}^{A r^{2}}
$$

for suitable constants $c \geq 0, A \geq 0$;

(c) $(X, \mathrm{~d}, \mathfrak{m})$ is infinitesimally Hilbertian according to the terminology introduced in [Gig12], i.e., the Cheeger energy $\mathrm{Ch}$ is a quadratic form.

As explained in [AGS11b], [AGS12], the quadratic form Ch canonically induces a strongly regular Dirichlet $\mathcal{E}$ form in $(X, \tau)$ (where $\tau$ is the topology induced by the distance $d$ ), as well as a Carré du champ $\Gamma: D(\mathcal{E}) \times D(\mathcal{E}) \rightarrow L^{1}(\mathfrak{m})$. Thus, we recover the basic setting of $(2.1)$ and we can identify $W^{1,2}(X, d, \mathfrak{m})$ with $\mathbb{V}$. In addition, $\mathrm{P}$ is conservative because of $(9.3)$ and the definition of $\mathrm{Ch}$ provides the approximation property

$$
\exists f_{n} \in \operatorname{Lip}(X) \cap L^{2}(\mathfrak{m}) \text { with } f_{n} \rightarrow f \text { in } L^{2}(\mathfrak{m}) \text { and }\left|\mathrm{D} f_{n}\right| \rightarrow \sqrt{\Gamma(f)} \text { in } L^{2}(\mathfrak{m})
$$

for all $f \in \mathbb{V}$.

The above discussions justify the following definition of $\operatorname{RCD}(K, \infty)$. It is not the original one given in [AGS11b] we mentioned at the beginning of this section, but it is more appropriate for our purposes; the equivalence of the two definitions is given in [AGS12].

Definition $9.1(\operatorname{RCD}(K, \infty)$ metric measure spaces). We say that $(X, d, \mathfrak{m})$, satisfying (a), (b), (c) above, is an $\operatorname{RCD}(K, \infty)$ space if: 
(a) the Dirichlet form associated to the Cheeger energy of $(X, d, \mathfrak{m})$ satisfies $\mathrm{BE}_{2}(K, \infty)$ according to Definition 6.1;

(b) any $f \in W^{1,2}(X, d, \mathfrak{m}) \cap L^{\infty}(\mathfrak{m})$ with $\|\Gamma(f)\|_{\infty} \leq 1$ has a 1-Lipschitz representative.

From [AGS11b, Lemma 6.7] we obtain that $\mathcal{E}$ is quasi-regular. We set throughout $\mathscr{A}$ be the class of Lipschitz functions with bounded support. It is easily seen that $\mathscr{A}$ is dense in $\mathbb{V}$.

Since both $(X, d)$ and $\mathbb{V}$ are separable, it is not difficult to exhibit a countable family $\mathscr{A}^{*} \subset \mathscr{A}$ such that (7.10) and (7.11) are satisfied: let $\left(x_{h}\right) \subset X$ be dense, and set $f_{h, k}:=$ $\left(d\left(x_{h}, \cdot\right)-k\right)^{-} \in \mathscr{A}$ for $h, k \in \mathbb{N}$; then, define

$$
\mathscr{B}:=\bigcup_{h, k=0}^{\infty}\left\{f_{h, k}\right\} \cup \bigcup_{h=0}^{\infty}\left\{g_{h}\right\},
$$

with $\left(g_{h}\right) \subset \mathscr{A}$ dense in $\mathbb{V}$. Then, defining $\mathscr{A}^{*}=\left\{f \in \mathscr{B}:\|\Gamma(f)\|_{\infty} \leq 1\right\} \subset \mathscr{A}$, since $\mathbb{R} \mathscr{A}^{*}=\mathscr{B}$ we obtain (7.10), while (7.11) follows easily by the fact that all functions $f_{h, k}$ belong to $\mathscr{A}^{*}$. Moreover, the distance $d_{\varepsilon}$ defined by (7.12) in Remark 7.2 coincides with $d$ : $d_{\mathscr{A}^{*}} \leq d$ is obvious, while $d \leq d_{\mathscr{A}^{*}}$ follows from taking $f=f_{h, k}$ in (7.12), with $x_{h}$ arbitrarily close to $x$ and $k$ larger than $d(x, y)$.

We discuss now the fine regularity properties of functions in $\mathbb{V}$, recalling some results developed in [AGS11a]. We start with the notion of 2-plan.

Definition 9.2 (2-plans). We say that a positive finite measure $\boldsymbol{\eta}$ in $\mathscr{P}(C([0, T] ; X))$ is a 2-plan if $\boldsymbol{\eta}$ is concentrated on $A C([0, T] ;(X, d))$ and the following two properties hold:

(a) $\iint_{0}^{T}|\dot{\eta}|^{2}(t) d t d \boldsymbol{\eta}(\eta)<\infty$;

(b) there exists $C \in[0, \infty)$ satisfying $\left(e_{t}\right)_{\#} \boldsymbol{\eta} \leq C \mathfrak{m}$ for all $t \in[0, T]$.

Accordingly, we say that $V: X \rightarrow \mathbb{R}$ is $W^{1,2}$ along 2-almost every curve if, for all $s \leq t$ and all 2-plans $\boldsymbol{\eta}$, the family of inequalities

$$
\int|V(\eta(s))-V(\eta(t))| d \boldsymbol{\eta}(\eta) \leq \iint_{s}^{t} g(\eta(r))|\dot{\eta}(r)| d r d \boldsymbol{\eta}(\eta), \quad \text { for all } s, t \in[0, T) \text { with } s \leq t
$$

holds for some $g \in L^{2}(\mathfrak{m})$. Since Lipschitz functions with bounded support are dense in $\mathbb{V}$, a density argument [AGS11a, Thm. 5.14] based on (9.4) provides the following result:

Proposition 9.3. Any $V \in \mathbb{V}$ is $W^{1,2}$ along 2-almost every curve. In addition, (9.5) holds with $g=\sqrt{\Gamma(V)}$.

Actually, a much finer result could be established (see [AGS11a, $\S 5]$ ), namely the existence of a representative $\tilde{V}$ of $V$ in the $L^{2}(\mathfrak{m})$ equivalence class, with the property that $\tilde{V} \circ \eta$ is absolutely continuous $\boldsymbol{\pi}$-a.e. $\eta$ for any 2-plan $\boldsymbol{\pi}$, with $\left|(\tilde{V} \circ \eta)^{\prime}\right| \leq \sqrt{\Gamma(V)}|\dot{\eta}|$ a.e. in $(0, T)$. However, we shall not need this fact in the sequel. Here we notice only that since $\chi_{B} \boldsymbol{\eta}$ is a 2-plan for any Borel set $B \subset C([0, T] ; X)$, it follows from $(9.5)$ with $g=\sqrt{\Gamma(V)}$ that

$$
|V(\eta(s))-V(\eta(t))| \leq \iint_{s}^{t} \sqrt{\Gamma(V)}(\eta(r))|\dot{\eta}(r)| d r, \quad \text { for } \boldsymbol{\eta} \text {-a.e. } \eta
$$

for all $s, t \in[0, T)$ with $s \leq t$.

Now, we would like to relate these known facts to solutions to the ODE $\dot{\eta}=\boldsymbol{b}_{t}(\eta)$. The first connection between 2-plans and probability measures concentrated on solutions to the ODE is provided by the following proposition. 
Proposition 9.4. Let $\boldsymbol{b}=\left(\boldsymbol{b}_{t}\right)$ be a Borel family of derivations with $|\boldsymbol{b}| \in L_{t}^{1}\left(L^{2}\right)$ and let $u \in L_{t}^{\infty}\left(L_{x}^{\infty}\right)$. Let $\boldsymbol{\eta}$ be concentrated on solutions to the $O D E \dot{\eta}=\boldsymbol{b}_{t}(\eta)$, with $\left(e_{t}\right)_{\#} \boldsymbol{\eta}=u_{t} \mathfrak{m}$ for all $t \in(0, T)$. Then $\boldsymbol{\eta}$ is a 2-plan.

Proof. The fact that $\boldsymbol{\eta}$ has bounded marginals follows from the assumption $u \in L_{t}^{\infty}\left(L_{x}^{\infty}\right)$. By Lemma 7.4 and the identification $d=d_{\mathscr{A}^{*}}, \boldsymbol{\eta}$ is concentrated on $A C([0, T] ;(X, d))$, with $|\dot{\eta}|(t) \leq\left|\boldsymbol{b}_{t}\right|(\eta(t)), \mathscr{L}^{1}$-a.e. in $(0, T)$ for $\boldsymbol{\eta}$-a.e. $\eta$. Thus,

$$
\iint_{0}^{T}|\dot{\eta}|^{2}(t) d t d \boldsymbol{\eta}(\eta) \leq \int_{0}^{T} \int\left|\boldsymbol{b}_{t}\right|^{2} u_{t} d \mathfrak{m} d t<\infty .
$$

We now focus on the case of a "gradient" and time-independent derivation $\boldsymbol{b}_{V}$ associated to $V \in \mathbb{V}$. Recall that in this case $\left|\boldsymbol{b}_{V}\right|^{2}=\Gamma(V) \mathfrak{m}$-a.e. in $X$.

Theorem 9.5. Let $V \in D(\Delta)$ with $\Delta V^{-} \in L^{\infty}(\mathfrak{m})$. Then, there exist weakly continuous solutions (in $[0, T)$, in duality with $\mathscr{A}) u \in L_{t}^{\infty}\left(L_{x}^{1} \cap L_{x}^{\infty}\right)$ to the continuity equation, for any initial condition $\bar{u} \in L^{1} \cap L^{\infty}(\mathfrak{m})$. In addition, if $\boldsymbol{\eta}$ is given by Theorem 7.6 (namely $\boldsymbol{\eta}$ is concentrated on solutions to the $O D E \dot{\eta}=\boldsymbol{b}_{V}(\eta)$ and $\left(e_{t}\right)_{\#} \boldsymbol{\eta}=u_{t}$ for all $\left.t \in(0, T)\right)$, then:

(a) $\boldsymbol{\eta}$ is concentrated on curves $\eta$ satisfying $|\dot{\eta}|(t)=\Gamma(V)^{1 / 2}(\eta(t))$ for a.e. $t \in(0, T)$;

(b) for all $s, t \in[0, T)$ with $s \leq t$, there holds

$$
V \circ \eta(t)-V \circ \eta(s)=\int_{s}^{t} \Gamma(V)(\eta(r)) d r, \quad \text { for } \boldsymbol{\eta} \text {-a.e. } \eta \text {. }
$$

Proof. The proof of the first statement follows immediately by Theorem 4.3 with $r=\infty$. Since

$$
\int_{s}^{t} \int \Gamma(V, f) u_{r} d \mathfrak{m} d r=\int f u_{t}-\int f u_{s} \quad \text { for all } s, t \in[0, T) \text { with } s \leq t
$$

for all $f \in \mathscr{A}$, we can use the density of $\mathscr{A}$ in $\mathbb{V}$ and a simple limiting procedure to obtain

$$
\int_{s}^{t} \int \Gamma(V) u_{r} d \mathfrak{m} d r=\int V u_{t}-\int V u_{s} \quad \text { for all } s, t \in[0, T) \text { with } s \leq t .
$$

If $\boldsymbol{\eta}$ is as in the statement of the theorem, since $\boldsymbol{\eta}$ is a 2-plan we can combine Proposition 9.3 and the inequality $|\dot{\eta}| \leq\left|\boldsymbol{b}_{V}\right|(\eta)$ stated in Lemma 7.4 to get

$$
\int V(\eta(t))-V(\eta(s)) d \boldsymbol{\eta}(\eta) \leq \iint_{s}^{t} \Gamma(V)^{1 / 2}(\eta(r))|\dot{\eta}|(r) d r d \boldsymbol{\eta}(\eta) \leq \iint_{s}^{t} \Gamma(V)(\eta(r)) d \boldsymbol{\eta}(\eta)
$$

for all $s, t \in[0, T)$ with $s \leq t$. Since $\left(e_{r}\right)_{\#} \boldsymbol{\eta}=u_{r} \mathfrak{m}$ for all $r \in[0, T)$, it follows that

$$
\int V u_{t}-\int V u_{s}=\int V(\eta(t))-V(\eta(s)) d \boldsymbol{\eta}(\eta) \leq \int_{s}^{t} \Gamma(V) u_{r} d \mathfrak{m} d r .
$$

Combining (9.7) and (9.8) it follows that all the intermediate inequalities we integrated w.r.t. $\boldsymbol{\eta}$ are actually identities, so that for $\boldsymbol{\eta}$-a.e. $\eta$ it must be $|\dot{\eta}|=\sqrt{\Gamma(V)} \circ \eta$ a.e. in $(0, T)$ and equality holds in (9.6). 
In particular, one could prove that $\boldsymbol{\eta}$ is a 2-plan representing the 2-weak gradient of $V$, according to [Gig12, Def. 3.7], where a weaker asymptotic energy dissipation inequality was required at $t=0$. Our global energy dissipation is stronger, but it requires additional bounds on the Laplacian.

We can also prove uniqueness for the continuity equation, considering just for simplicity still the autonomous version.

Theorem 9.6. Let $V \in D(\Delta)$ with $\Delta V^{-} \in L^{\infty}(\mathfrak{m})$. Then the continuity equation induced by $\boldsymbol{b}_{V}$ has existence and uniqueness in $L_{t}^{\infty}\left(L_{x}^{1} \cap L_{x}^{\infty}\right)$ for any initial condition $\bar{u} \in L^{1} \cap L^{\infty}(\mathfrak{m})$.

Proof. We already discussed existence in Theorem 9.5. For uniqueness, we want to apply Theorem 5.4 with $q=2$ and $r=s=4$ (which provides uniqueness in the larger class $L^{2} \cap L^{4}(\mathfrak{m})$ ). In order to do this we need only to know that (4.3) holds (this follows by conservativity of $\mathrm{P}$ and $\mathrm{BE}_{2}(K, \infty)$ ), that $L^{4}-\Gamma$ inequalities hold in $\operatorname{RCD}(K, \infty)$ spaces (this follows by $\mathrm{BE}_{2}(K, \infty)$ thanks to Corollary 6.3$)$ and that the deformation of $\boldsymbol{b}_{V}$ is of type $(4,4)$ (this follows by Theorem 6.7).

\section{References}

[AK00] L. Ambrosio and B. Kirchheim, Currents in metric spaces. Acta Mathematica, 185 (2000), 1-80.

[Amb04] L. Ambrosio, Transport equation and Cauchy problem for BV vector fields. Invent. Math. 158 (2004), 227-260.

[Amb08] L. Ambrosio, Transport equation and Cauchy problem for non-smooth vector fields. Lecture Notes in Mathematics "Calculus of Variations and Non-Linear Partial Differential Equations" (CIME Series, Cetraro, 2005) 1927, B. Dacorogna, P. Marcellini eds., 2-41, 2008.

[AC08] L. Ambrosio and G. Crippa, Existence, uniqueness, stability and differentiability properties of the flow associated to weakly differentiable vector fields. Lecture Notes of the Unione Matematica Italiana 5, Springer, 2008.

[AF09] L. Ambrosio and A. Figalli, On flows associated to Sobolev vector fields in Wiener spaces: an approach à la DiPerna-Lions. J. Funct. Anal. 256 (2009), 179-214.

[AGS05] L. Ambrosio, N. Gigli, and G. Savaré, Gradient flows in metric spaces and in the space of probability measures. Lectures in Mathematics ETH Zürich, Birkhäuser Verlag, Basel, 2005.

[AGS11a] L. Ambrosio, N. Gigli, and G. Savaré, Calculus and heat flow in metric measure spaces and applications to spaces with Ricci bounds from below. Inventiones Mathematicae, 195 (2014), 289-391.

[AGS11b] L. Ambrosio, N. Gigli, and G. Savaré, Metric measure spaces with Riemannian Ricci curvature bounded from below. Arxiv 1109.0222 (2011).

[AGS12] L. Ambrosio, N. Gigli, and G. Savaré, Bakry-Émery curvature-dimension condition and Riemannian Ricci curvature bounds. ArXiv 1209.5786 (2012). 
[AMS13] L. Ambrosio, A. Mondino, and G. Savaré, On the Bakry-Émery condition, the gradient estimates and the Local-to-Global property of $\mathrm{RCD} *(K, N)$ metric measure spaces. ArXiv 1309.4664 (2013).

[ASZ09] L. Ambrosio, G. Savaré, and L. Zambotti, Existence and stability for Fokker-Planck equations with log-concave reference measure. Probab. Theory Relat. Fields $\mathbf{1 4 5}$ (2009), 517-564.

[Bak85] D. Bakry, Transformations de Riesz pour les semi-groupes symétriques. II. Étude sous la condition $\Gamma_{2} \geq 0$. Séminaire de probabilités, XIX, 1983/84, Lecture Notes in Math., vol. 1123, Springer, Berlin, 1985, pp. 145-174.

[Bak94] D. Bakry, On Sobolev and logarithmic Sobolev inequalities for Markov semigroups. New trends in stochastic analysis (Charingworth, 1994), World Sci. Publ., River Edge, NJ, 1997, 43-75.

[Bak94b] D. Bakry, L'hypercontractivité et son utilisation en théorie des semigroupes. Lectures on probability theory (Saint-Flour, 1992), Lecture Notes in Math., vol 1581, Springer, Berlin, 1994.

[BGL13] D. Bakry, I. Gentil and M. Ledoux, Analysis and Geometry of Markov Diffusion Operators. Springer Verlag, 2013.

[Ba12] D. Bate, Structure of measures in Lipschitz differentiability spaces. ArXiv $1208.1954(2012)$

[BH91] N. Bouleau and F. Hirsch, Dirichlet forms and analysis on Wiener sapces. De Gruyter studies in Mathematics 14, De Gruyter, 1991.

[BM95] M. Biroli and U. Mosco, A Saint-Venant principle for Dirichlet forms on discontinuous media. Ann. Mat. Pura Appl. 169 (1995), 125-181.

[Bog98] V.I. Bogachev, Gaussian measures, Mathematical Surveys and Monographs, vol. 62, American Mathematical Society, Providence, RI, 1998.

[Bog10] Differentiable measures and the Malliavin calculus, Mathematical Surveys and Monographs, vol. 164, American Mathematical Society, Providence, RI, 2010.

[CP96] I. Capuzzo Dolcetta and B. Perthame, On some analogy between different approaches to first order PDE's with nonsmooth coefficients, Adv. Math. Sci Appl. 6 (1996), 689-703.

[DFR13] G. Da Prato, F. Flandoli, and M. Röckner, Uniqueness for continuity equations in Hilbert spaces with weakly differentiable drift, ArXiv 1305.7148 (2013).

[DL89] R. J. DiPerna and P.-L. Lions, Ordinary differential equations, transport theory and Sobolev spaces, Invent. Math. 98 (1989), 511-547.

[DP04] G. Da Prato, Kolmogorov equations for stochastic PDEs. Advanced Courses in Mathematics. CRM Barcelona, Birkhäuser, 2004. 
[FOT11] M. Fukushima, Y. Oshima, and M. Takeda, Dirichlet forms and symmetric Markov processes. Extended ed., De Gruyter Studies in Mathematics, vol. 19, Walter de Gruyter \& Co., Berlin, 2011.

[Gig12] N. Gigli, On the differential structure of metric measure spaces and applications. ArXiv 1205.6622 (2012).

[Gig13] N. Gigli, The splitting theorem in non-smooth context. ArXiv 1302.5555 (2013).

[GB14] N. Gigli, H. Bangxian, The continuity equation on metric measure spaces. To appear in Calc. Var. Partial Differential Equations (2014).

[Gig14] N. Gigli, Work in progress, 2014.

[Gri99] A. Grigor'yan, Analytic and geometric background of recurrence and non-explosion of the Brownian motion on Riemannian manifolds. Bull. Am. Math. Soc. 36, pp. 135-249 (1999).

[KR13] A. V. Kolesnikov and M. Röckner, On continuity equations in infinite dimensions with non-Gaussian reference measure, ArXiv 1303.7184 (2013).

[Led00] M. Ledoux, The geometry of Markov diffusion generators. Ann. Fac. Sci. Toulouse Math. (6) 9 (2000), 305-366, Probability theory.

[LV09] J. Lott and C. Villani, Ricci curvature for metric-measure spaces via optimal transport. Ann. of Math. (2) 169 (2009), 903-991.

[MWZ05] E. Mayer-Wolf and M. Zakai, The divergence of Banach space valued random variables on Wiener space, Probab. Theory Related Fields 132 (2005), 291-320.

[Sav13] G. Savaré, Self-improvement of the Bakry-Émery condition and Wasserstein contraction of the heat flow in $R C D(K, \infty)$ metric measure spaces, Discrete Contin. Dyn. Syst. (A), 34 (2014), 1641-1661.

[Sc13] A. Schioppa, Derivations and Alberti representations. ArXiv 1311.2439 (2013).

[Sho97] R. E. Showalter, Monotone operators in Banach space and nonlinear partial differential equations, American Mathematical Society, Providence, RI, 1997.

[Smi94] S.K. Smirnov, Decomposition of solenoidal vector charges into elementary solenoids and the structure of normal one-dimensional currents. St. Petersburg Math. J., 5, pp. 841-867, 1994.

[Ste70] E. Stein, Topics in harmonic analysis related to the Littlewood-Paley theory. Annals of Mathematics Studies, 63, Princeton University Press, Princeton, N.J., 1970.

[Sto10] P. Stollmann, A dual characterization of length spaces with application to Dirichlet metric spaces, Polska Akademia Nauk. Instytut Matematyczny. Studia Mathematica 198 (2010), no. 3, 221-233.

[Stu95] K.T. Sturm, Analysis on local Dirichlet spaces. II. Upper Gaussian estimates for the fundamental solutions of parabolic equations, Osaka Journal of Mathematics 32 (1995), no. 2, 275-312. 
[Stu06a] _ On the geometry of metric measure spaces I. Acta Math. 196 (2006), 65-131.

[Stu06b] , On the geometry of metric measure spaces II. Acta Math. 196 (2006), $133-177$.

[Tre13] D. Trevisan, Lagrangian flows driven by BV fields in Wiener spaces. ArXiv 1310.5655 (2013).

[Vil09] C. Villani, Optimal transport. old and new. Grundlehren der Mathematischen Wissenschaften 338, Springer-Verlag, Berlin, 2009.

[We00] N. Weaver, Lipschitz algebras and derivations. II. Exterior differentiation. J. Funct. Anal., 178 (2000), pp. 64-112.

[WW09] G. Wei and W. Wylie, Comparison geometry for the Bakry-Emery Ricci tensor. J. Differential Geom. 83 (2009), no. 2, pp. 377-405.

[Yos80] K. Yosida, Functional analysis. Sixth edition. Grundlehren der Mathematischen Wissenschaften, 123. Springer-Verlag, Berlin-New York, 1980. 